\title{
Thermal Detection of Embedded Tumors using Infrared Imaging
}

\author{
Thesis submitted to the faculty of the \\ Virginia Polytechnic Institute and State University \\ in partial fulfillment of the requirements for the degree of
}

\section{MASTER OF SCIENCE}

in

Mechanical Engineering

APPROVED:

Dr. Elaine P. Scott, Chair

Dr. Brian Vick, Committee Member

Dr. Thomas E. Diller, Committee Member

August 23, 2004

Blacksburg, Virginia

Keywords: Genetic Algorithms, Numerical Heat Transfer, Optimization, Tumor 


\title{
Thermal Detection of Embedded Tumors using Infrared Imaging
}

\author{
Manu Mital, M.S. \\ Virginia Polytechnic Institute and State University, 2004 \\ Advisor: Dr. Elaine P. Scott
}

\begin{abstract}
Breast cancer is the most common cancer among women. Statistics released by the American Cancer Society (1999) show that every 1 in 8 women in the United States is likely to get breast cancer during her lifetime. Thermography, also known as thermal or infrared imaging, is a procedure to determine if an abnormality is present in the breast tissue temperature distribution, which may indicate the presence of an embedded tumor. In the year 1982, the United States Food and Drug Administration (FDA) approved thermography as an adjunct method of detecting breast cancer, which could be combined with other established techniques like mammography. Although thermography is currently used to indicate the presence of an abnormality, there are no standard protocols to interpret the abnormal thermal images and determine the size and location of an embedded tumor. This research explores the relationship between the physical characteristics of an embedded tumor and the resulting temperature distributions on the skin surface. Experiments were conducted using a resistance heater that was embedded in agar in order to simulate the heat produced by a tumor in the biological tissue. The resulting temperature distribution on the surface was imaged using an infrared camera. In order to estimate the location and heat generation rate of the source from these temperature distributions, a genetic algorithm was used as the estimation method. The genetic algorithm utilizes a finite difference scheme for the direct solution of Pennes bioheat equation. It was determined that a genetic algorithm based approach is well suited for the estimation problem since both the depth and the heat generation rate of the heat source were accurately predicted. Thermography can prove to be a valuable tool in locating tumors if combined with such an algorithm.
\end{abstract}




\section{Acknowledgements}

I would like to thank Dr. Elaine P. Scott for her support and for serving as my advisor. I would also like to thank Dr. Brian Vick and Dr. Thomas E. Diller for serving on my advisory committee. I would like to thank my sister Mala Mital for providing constant support and encouraging me to pursue education. I am also tremendously grateful for the help and assistance from my labmates Ying-Feng Pang, Max Savransky, Marie Madden and F. Scott Gayzik. Finally, I would like to thank Virginia Tech Department of Mechanical Engineering for providing me with graduate student assistantship funding that made it possible for me to pursue this degree.

MANU MITAL

Virginia Polytechnic Institute and State University

August 2004 


\section{List of Tables}

Table 5.1 Property values used for Evaluation of the Model ........................................ 39

Table 5.2 Parameters used for different experimental runs .......................................... 45

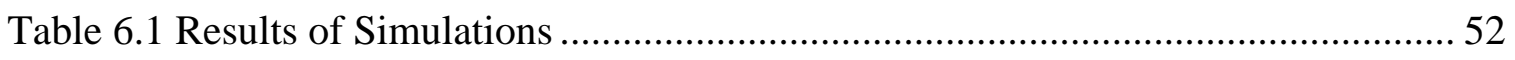

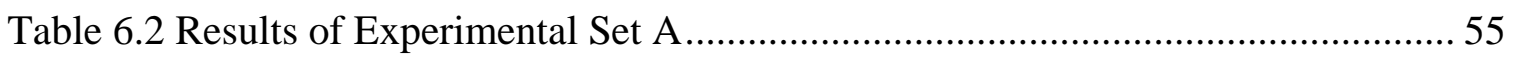

Table 6.3 Results of Experimental Set B ................................................................. 58

Table I.1 Specifications for the PV320 Infrared Camera ........................................... 112 


\section{List of Figures}

Fig. 2.1 A genetic search technique starts out with a population of initial points............ 14

Fig. 3.1 Finite difference scheme in cylindrical coordinates ...................................... 17

Fig. 3.2 Discretization of two-dimensional domain in cylindrical coordinates (Vick, $2003)$

Fig. 3.3 A control volume in the two-dimensional domain (Vick, 2003)...................... 20

Fig. 3.4 Representation of the ADI method in the two dimensional domain .................. 24

Fig. 3.5 A flowchart of the estimation program.......................................................... 25

Fig. 3.6 The variation of sum of squares function with tumor height and heat generation

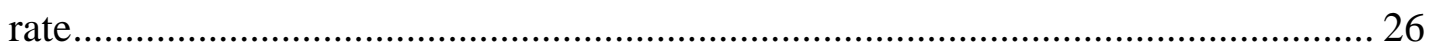

Fig. 3.7 Dimensionless sensitivity of temperature to tumor size for different tumor locations 30

Fig. 3.8 Dimensionless sensitivity of temperature to tumor location for different tumor sizes....... 30

Fig. 3.9 Dimensionless sensitivity of temperature to tumor location and radius.............. 31

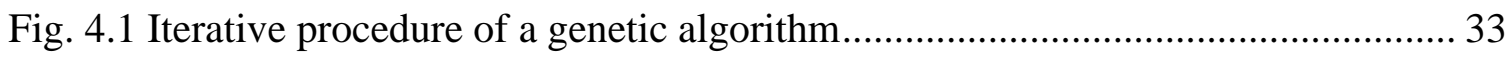

Fig. 4.2 Roulette wheel concept of selection ............................................................ 35

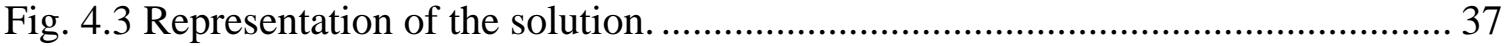

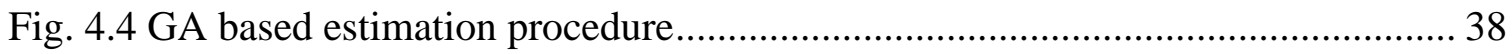

Fig. 5.1 Schematic of the experimental setup ........................................................... 42

Fig. 5.2 Photograph of the experimental setup .......................................................... 43

Fig. 5.3 Figure showing the (a) photograph and (b) dimensions of the cylinder and base

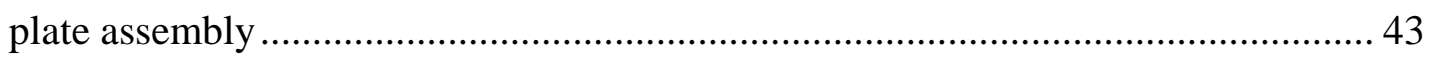

Fig. 5.4 The resistance heater used as the heat source in the experiment ....................... 44

Fig. 5.5 The Infrared camera used in the experiment .............................................. 44

Fig. 5.6 The parameters used for different experimental runs and the limits of steady-state detection of the heater assuming a mimimum temperature rise of 0.5 degrees on the surface 46

Fig. 5.7 Infrared camera calibration using thermocouple ........................................ 47

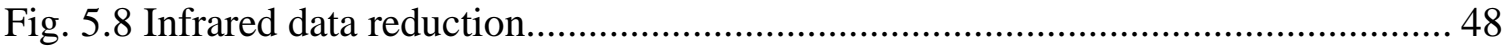


Fig. 5.9 A thermal image showing the surface of the cylinder and some radial lines along

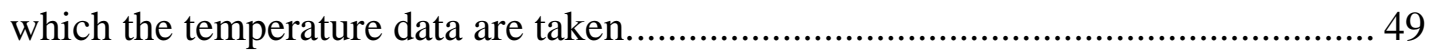

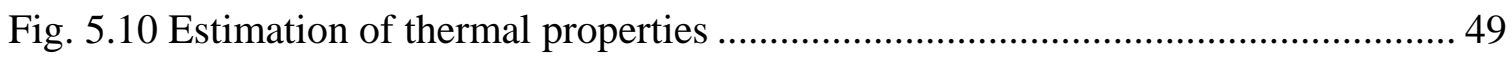

Fig. 6.1 3D ANSYS model showing the temperature distributions in a cylinder with an

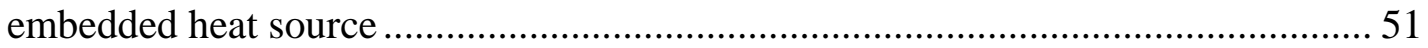

Fig. 6.2 Temperature profiles obtained using finite difference model and ANSYS

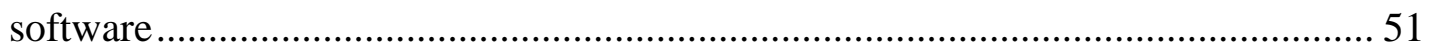

Fig. 6.3 Plot showing simulated data and the resulting temperature distribution predicted using estimation program for cases a,b and c.

Fig. 6.4 Result of Experiment A1 showing experimental data and predicted temperature profiles 56

Fig. 6.5 Result of Experiment A2 showing experimental data and predicted temperature profiles 56

Fig. 6.6 Result of Experiment A3 showing experimental data and predicted temperature profiles 57

Fig. 6.7 Result of Experiment A4 showing experimental data and predicted temperature profiles ..... 57

Fig. 6.8 Result of Experiment B1 showing experimental data and predicted temperature profiles 59

Fig. 6.9 Result of Experiment B2 showing experimental data and predicted temperature profiles 59

Fig. 6.10 The effect of convection coefficient on model output for experiment A1 ....... 60

Fig. 6.11 The effect of convection coefficient on model output for experiment B2 ........ 61

Fig. 6.12 The effect of thermal conductivity on model output for experiment B2 62 


\title{
Nomenclature
}

\author{
E Total emitted radiance, $\mathrm{W} / \mathrm{m}^{2}$ \\ $\bar{h} \quad$ Average convection coefficient, $\mathrm{W} / \mathrm{m}^{2}-\mathrm{K}$ \\ k Thermal conductivity, W/m-K \\ Q Heat generation, $\mathrm{W} / \mathrm{m}^{3}$ \\ $\mathrm{t}$ Time, $\mathrm{s}$ \\ S Sum of squares \\ $\mathrm{T} \quad$ Temperature, ${ }^{\circ} \mathrm{C}$ \\ X Sensitivity coefficient matrix \\ $\beta \quad$ Model parameter vector \\ $\rho \quad$ Density \\ $\sigma \quad$ Stefan-Boltzmann constant, $\mathrm{W} \mathrm{m}^{-2} \mathrm{~K}^{-4}$ \\ $\omega \quad$ Blood perfusion term
}

\section{Subscripts}
a Arterial
b Blood
m Metabolic

\section{Superscripts}

$\begin{array}{ll}\mathrm{k} & \text { Iteration index } \\ \mathrm{T} & \text { Transpose }\end{array}$




\section{Table of Contents}

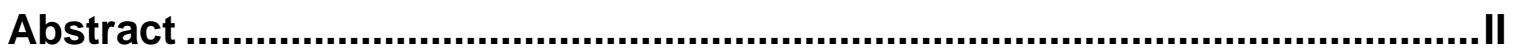

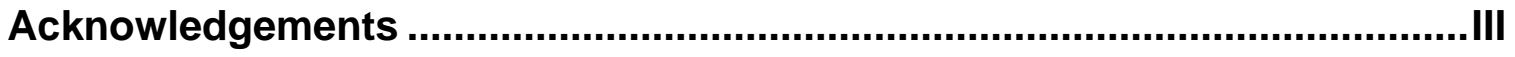

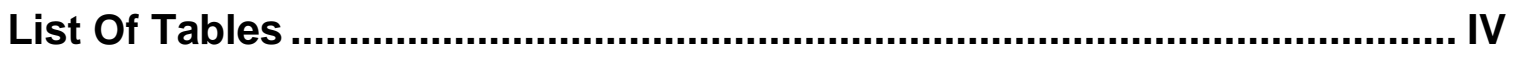

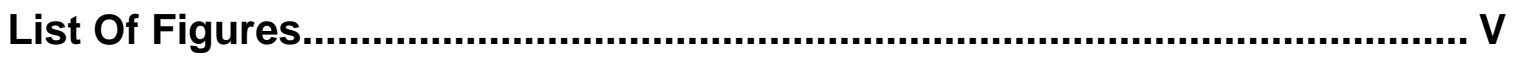

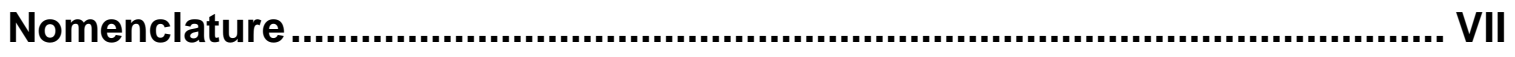

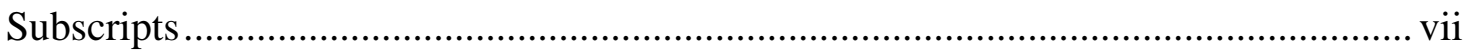

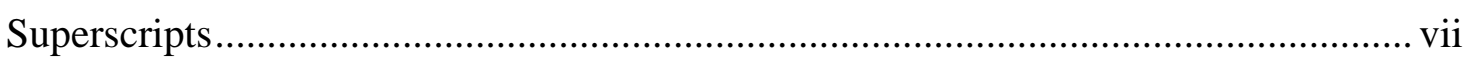

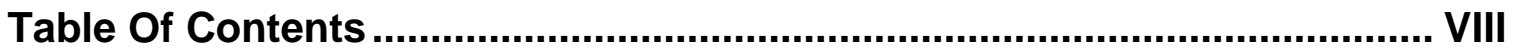

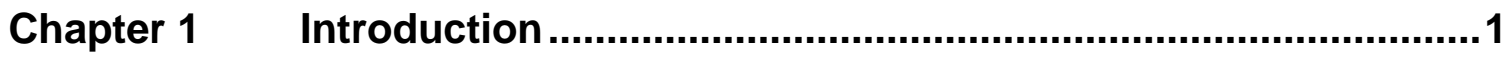

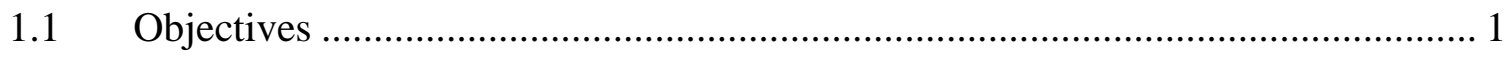

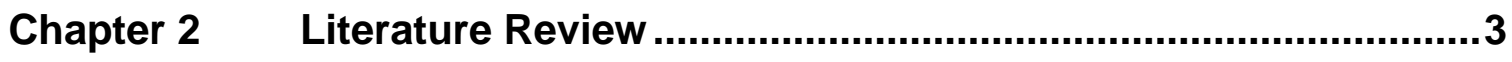

2.1 Medical Thermal Imaging: Past And Present …………............................................... 3

2.1.1 Causes For Failure Of Thermal Imaging In The Past.............................................. 3

2.1.2 Thermal Imaging In Relation To Existing Methods ................................................. 5

2.1.3 Future Prospects Of Medical Thermal Imaging....................................................... 7

2.2 How Is Thermal Imaging Performed? ................................................................... 8

2.3 Modeling Heat Transfer In Tumors ............................................................................ 9

2.3.1 Biology Of Tumor Growth ............................................................................ 9

2.3.2 The Bioheat Equation ................................................................................... 10

2.4 Temperature Distributions Due To A Tumor ………………………………………... 11 
2.5 Genetic Algorithms (GAs) .................................................................................... 13

2.5.1 Background And Description ......................................................................... 13

2.5.2 Genetic Algorithms In Comparison To Traditional Methods................................ 14

2.5.3 Recent Applications Of Genetic Algorithms ................................................... 15

Chapter $3 \quad$ Numerical Model And Estimation Methodology....................17

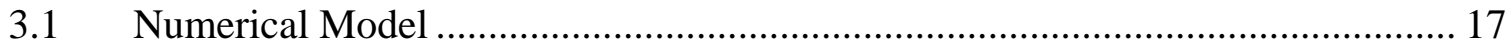

3.1.1 Finite Difference Scheme .................................................................... 18

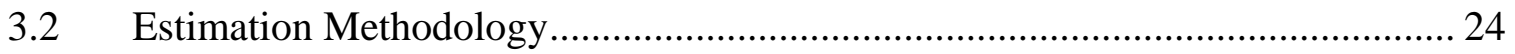

3.2.1 The Objective Function................................................................................. 25

3.2.2 Gradient Based Parameter Estimation Approach ........................................... 26

3.2.3 Sensitivity Coefficients............................................................................. 29

3.2.4 Parameter Estimation Using Genetic Algorithms ........................................... 32

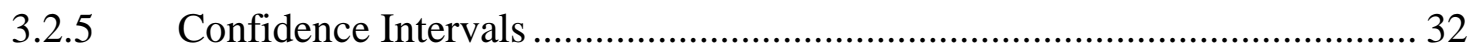

Chapter $4 \quad$ Genetic Algorithms (Gas) ..................................................33

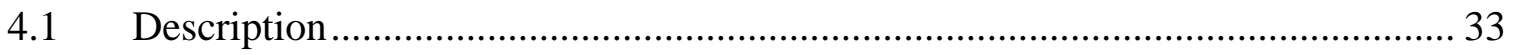

4.1.1 Encoding ..................................................................................................... 34

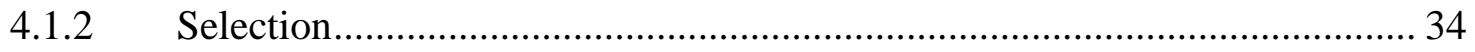

4.1.3 Genetic Modifications................................................................................ 35

4.2 Genetic Algorithms In Detection Of Embedded Tumors ...................................... 36

Chapter $5 \quad$ Numerical And Experimental Validation ..............................39

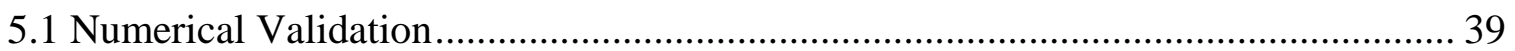

5.1.1 Validation Of Finite Difference Model........................................................... 39

5.1.2 Validation Of Parameter Estimation Program ................................................. 39

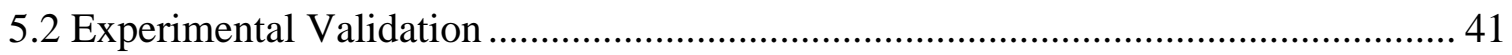

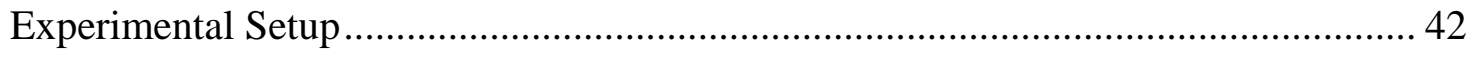


Chapter 6 Numerical And Experimental Results.........................................50

6.1 Numerical Model Validation Results.................................................................... 50

6.2 Parameter Estimation Program Validation Results........................................................ 52

6.3 Results Of Experiments ……………………………………................................ 54

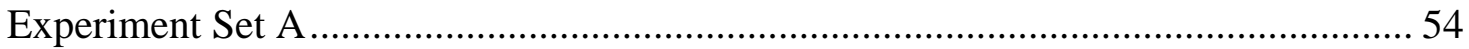

Experiment Set B …………………………………...................................... 58

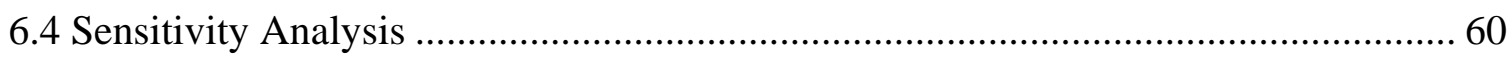

Chapter 7 Conclusions And Recommendations ......................................63

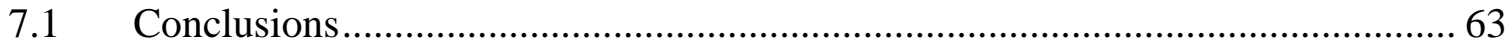

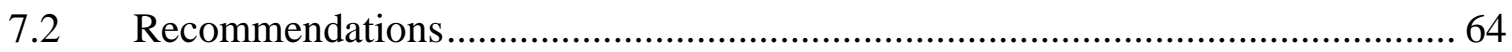

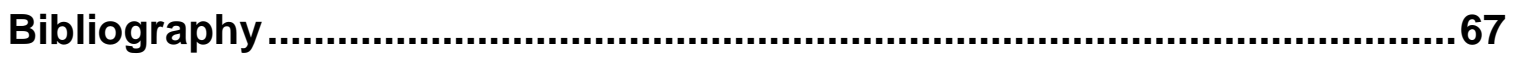

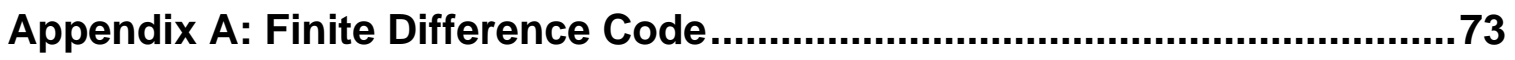

Appendix B: Alternating-Direction Implicit Solver.......................................83

Appendix C: Tridiagonal Matrix Algorithm (Thomas Algorithm) .................87

Appendix D: Box-Kanemasu Method Flowchart .........................................89

Appendix E

E1 Box-Kanemasu Method Code For Parameter Estimation ............................................. 90

E2 Box-Kanemasu Method Code For Estimating Convection Coefficient ........................ 94 
Appendix F: Genetic Algorithm Code

Appendix G: Infrared Data Reduction Program ........................................106

Appendix H: Estimation Program Flowchart ............................................111

Appendix I: Infrared Camera Specifications.............................................112

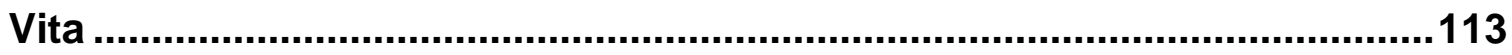




\section{Chapter 1 Introduction}

The temperature of the human body on the surface of the skin depends on the metabolic activity, the blood flow and the temperature of the surroundings. Any abnormality in the tissue, such as the presence of a tumor, alters the normal temperature on the skin surface due to increased metabolic activity of the tumor. Therefore, abnormal skin temperature profiles can be used as indicators of a disease.

The earliest known use of thermal diagnostic of diseases by measuring the surface temperature of the skin is found as early as 480 B.C. in works of Hippocrates. Mud slurry was spread over the skin of the patient and allowed to dry. The areas on the skin that were found to dry earlier than others were thought to show the presence of a disease.

In the 1950s, the US military started research into infrared monitors to detect the movement of troops at night. Lawson (1956) was the first to propose the use of thermographic detection of breast cancer, when he observed that the local temperatures of the skin over a tumor were significantly higher (about $2-3$ degrees) than normal skin temperatures. Lawson and Chughtai (1963) demonstrated that the regional temperature difference over an embedded tumor was due to convection effects associated with increased blood perfusion, and increased metabolism around the tumor. Gershon-Cohen (1965) at the Albert Einstein Medical Center, introduced thermal imaging in United States. He reported 4,000 cases with a false-positive rate of 6 percent. A paper published by the Department of Health Education in the year 1972 identified the position of thermal imaging as being beyond its experimental stage.

\subsection{Objectives}

The specific objectives of this research were to:

1. develop a generic numerical model that can be used to incorporate tumors embedded in the tissue, such as tumors in the breast 
2. develop an estimation procedure for locating embedded tumors and estimating their heat generation rate

3. develop a simple experimental procedure to test the model and the estimation program, and evaluate the effect of model parameters on the results.

This document explains how the objectives of this work were addressed. In Chapter 2, a literature review is presented to provide insights into some of the key topics related to this research. These topics include medical thermal imaging, the biological basis of tumor growth, and the temperature profiles in a medium due to the presence of an embedded heat source. A review of genetic algorithms is provided since it is used as the main estimation method in this research. In Chapter 3, a finite difference scheme based on the Pennes bioheat equation is developed. The usefulness of both gradient-based estimation method and genetic algorithms are evaluated. Chapter 4 describes genetic algorithms and how they are used in the current research. A detailed description is then given in Chapter 5 about the experimental setup and the data acquisition system. In Chapter 6, the temperature profiles obtained from the experiments are discussed, and compared with temperature profiles obtained from finite difference scheme based on Pennes bioheat equation. The estimation program is validated using both simulated and experimental data. Finally, the conclusions and recommendations as a result of this study are presented in Chapters 7 and 8, respectively. 


\section{Chapter 2 Literature Review}

Several areas of scientific literature provide crucial insights into the methods used in this research. The main areas include medical thermal imaging, the biological basis of tumor growth, and the temperature profiles in a medium due to the presence of an embedded heat source. A literature review of genetic algorithms is also provided since it is later used as the main estimation method. This literature provides a sufficient basis for understanding the methods used in the current research.

\subsection{Medical Thermal Imaging: Past and Present}

This section briefly analyzes the causes for failure of thermal imaging as a medical diagnostic tool, the current status of thermal imaging in relation to other detection techniques, and the future prospects of thermal imaging as a tool for detecting cancer.

\subsubsection{Causes for Failure of Thermal Imaging in the Past}

In 1973, The Breast Cancer Detection and Demonstration Projects (BCDDP) were initiated at 29 locations around the United States, with a total participation of about 280,000 women. The National Cancer Institute (NCI) and the American Cancer Society jointly established these projects for screening a large number of women with breast cancer. The purpose of the screening process is to identify those with particular distinguishing characteristics that may arouse suspicion about the presence of a disease. Diagnosis refers to the process of identifying the nature or cause of the disease. The three techniques that were used in screening are physical examination, mammography and thermography. 
During the beginning of these projects, there was an interest in evaluating the potential of thermography as a substitute for mammography in screening for cancer. It was found that only 41 percent of the breast cancers were detected when using thermography, during the first screening. The remaining thermographic interpretations were either abnormal or unknown. In 1978, thermography was discontinued as a routine test. In the final recommendation, the working group stated that "thermal imaging does not appear to be suitable as a substitute for mammography for routine screening in the BCDDP". However, it was also recommended that dismissal of thermal imaging should not be interpreted to indicate the future of this technique. It was realized that more research is needed to develop thermal imaging as an adjunct to mammography in detecting cancer.

Joann Haberman, who participated in these projects, reported that one of the shortcomings of these projects was the inadequacy of training provided to the technicians and radiologists. Out of the 29 demonstration project centers, only 5 centers had availability of expertise in thermal imaging. The remaining centers had technicians that were either inadequately trained in thermal imaging techniques or had no experience at all. It was after 18 months that the project had begun, NCI established training center for the technicians and radiologists to learn proper techniques of thermal imaging.

Another factor that contributed to the failure of thermal imaging was the lack of control over the physical surroundings where the imaging was performed. It was reported that many of the experiments were performed in mobile vans that had inadequate heating and cooling capabilities. Images were taken in an uncontrolled thermal environment that severely degraded their quality.

Before the 1980s, there were no standard sets of rules for the reading of thermal images to detect cancer. The thermal images were interpreted based on the judgment of the radiologists to determine if an abnormality was present.

Thus, the lack of expertise in obtaining thermal images and their interpretation, absence of high-resolution infrared cameras, and inadequately performed experiments, all contributed towards failure in establishing thermography as a reliable technique in detection of cancer. 


\subsubsection{Thermal Imaging in Relation to Existing Methods}

Apart from physical examination, some other methods for detection of breast cancer include Ultrasound, Mammography, Magnetic Resonance Imaging (MRI) and Microwave Thermography. This section discusses these common techniques and their strengths and drawbacks.

\section{Ultrasound}

Ultrasound is one of the first exploratory tools that is used for evaluating a lump. Since the tissue of the body is inhomogeneous, it reflects and scatters pulses of highfrequency sound directed at it (Mottley, 1995). Ultrasound uses a pulse-echo mechanism in which high-energy sound waves are directed into the body and the echoes created by the interaction of the target and the ultrasound beam are received by transducers (Thomenius, 2003). It can distinguish between a cyst and a solid lump. Solid lumps require further examination in order to determine the presence of cancer. According to Warren (2001), breast ultrasound is low cost and radiation free and can be useful if combined with family history, but it is not recommended as a stand alone technique for breast cancer detection. This is due to its low sensitivity for ductal microcalcifications in the breast.

\section{Mammography}

Mammography is currently the most reliable technique for detecting early-stage breast cancer (Yaffe, 1995). It uses high-energy x-ray beams that can penetrate through most of the soft material in the human body. The image is captured on the film located opposite to the projector. According to U.S. Food and Drug Administration (FDA), mammography can detect 85 percent of all breast cancers, several years before a lump can be noticed. Warren (2001) has compiled some important advantages and disadvantages of mammography as a screening method. The advantages include low cost of examination, acceptable sensitivity and specificity. The disadvantages include ionizing radiation which is a potential carcinogen and increases the risk of cancer, compression of 
the breast tissue in order to maintain the quality of the images, difficulty of detection in radiographically dense breasts, and need for highly skilled interpretation.

\section{Magnetic Resonance Imaging (MRI)}

The principle of MRI is based on the random distribution of protons that possess magnetic properties. Hydrogen nuclei are commonly used, although many other nuclei like ${ }^{2} \mathrm{H},{ }^{13} \mathrm{C},{ }^{19} \mathrm{~F},{ }^{23} \mathrm{Na},{ }^{31} \mathrm{P}$ and ${ }^{39} \mathrm{~K}$ may also be used for the purpose of imaging (Schaefer, 2003). Once the body is placed inside cylindrical magnets, MRI creates a steady state within the body by exposure to a steady magnetic field that has a magnitude of approximately about 1-2 Tesla (30,000 times stronger than the earth's magnetic field). The body is then exposed to radio waves to change the steady-state direction of the protons. Exposure to radio waves is then stopped and the electromagnetic transmissions from the body are captured at a given frequency. The transmitted signal is used to create images of the body.

Hata et al. (2004) conducted a study on 183 breast cancer patients in order to compare the techniques of MRI with mammography and ultrasound. The MRI included the use of a contrasting agent. The breast cancer detection rates using MRI, mammography and ultrasound were found to be 93.7, 84.6 and 97.3 percent respectively.

Lee et al. (2004) have identified several problems with MRI. The specificity of MRI is lower than its sensitivity which means that there are high levels of false-positive results. Another drawback is the high cost of the MRI examination and the follow-up costs. Although MRI does not involve any exposure to harmful radiations, it is potentially unsafe if there are any metallic implants in the body. Any metal objects would interfere with the magnetic field during the examination. The use of a contrast agent makes it an invasive procedure.

\section{Microwave Thermography}

Microwave thermography uses the radiation emitted from the body in the microwave region, approximately 0.001 to $0.3 \mathrm{~m}$ in wavelength. An antenna to receive the microwave radiation is placed in either in direct contact or at a short distance from the skin surface. This is done to minimize the loss at the interface of tissue and air. 
Barrett and Myers (1975) reported a temperature sensitivity of about $0.1^{\circ} \mathrm{C}$ and a spatial resolution of 0.01 by $0.02 \mathrm{~m}$ in sensing temperature distribution below the skin surface in human and animal tissue. They used microwave receivers to measure the microwave radiation emitted from subcutaneous tissues located at depths of several centimeters within the body.

Barrett et. al (1980) performed a breast cancer detection study on about 5000 female patients. Using microwave thermography, they collected data with radiation wavelengths 0.091 and $0.230 \mathrm{~m}$. At $0.230 \mathrm{~m}$ wavelengths, the true-positive and truenegative detection rates were found to be comparable to those of infrared thermography (about 0.7). From this study it was concluded that if microwave and infrared thermography are used together for screening purposes, then the true-positive and truenegative detection rates can both be as high as 0.9 , thus reducing the number of patients that need to be exposed to X-rays.

\subsubsection{Future Prospects of Medical Thermal Imaging}

Thermography promises to be a reliable adjunct technique in early cancer detection that can be combined with other established techniques like mammography. It still remains an adjunct technique since it cannot detect microcalcifications (mineral deposits) in the breast that can be seen only by mammography.However, it offers a means that is non-invasive and completely free of side effects such as exposure to harmful ionizing radiation. According to Amalu (2003), the abnormality in thermal images is the earliest known indicator (8-10 years in advance) for the possible development of breast cancer in the future. However, as mentioned earlier, it is not currently being used as a diagnostic tool that can identify and find the location of the tumor.

The Fraunhofer Institute for Applied Solid State Physics (IAF) has developed sensors that have a particularly high temperature resolution. The new sensors can measure differences in temperature up to 0.005 degrees. The development of highresolution digital infrared imaging, computerized image storage and assessment, and sophisticated technologies for image enhancement and analysis, offers renewed hope in thermography as a means of detecting cancer. The problems encountered with first 
generation thermal imaging systems such as unacceptable detector sensitivity, thermal drift, calibration, analog interface, etc. have been worked out in the last few decades.

\subsection{How is Thermal Imaging Performed?}

All objects with a temperature above absolute zero (-273 K) emit infrared radiation from their surface. The Stefan-Boltzmann law, also known as Stefan's law, states that the total energy radiated per unit surface area of a blackbody in unit time (blackbody irradiance), is directly proportional to the fourth power of its absolute temperature. This law can be mathematically expressed as:

$$
E=\sigma T^{4}
$$

where

$\mathrm{E}=$ total emitted radiance in $\mathrm{W} / \mathrm{m}^{2}$

$\sigma=5.6697 \times 10-8 \mathrm{~W} \mathrm{~m}^{-2} \mathrm{~K}^{-4}$ (Stefan-Boltzmann constant)

$\mathrm{T}=$ absolute temperature of the emitting material in Kelvin.

In order to maintain a constant temperature within the human body, the excess heat produced during metabolic activity is dissipated in part, in the form of infrared radiation. The wavelength of the radiation that leaves the surface of the skin at any given point is proportional to the local temperature of the skin at that point. Infrared rays are found in the electromagnetic spectrum within the wavelengths of 0.75 micron - $1 \mathrm{~mm}$, and the human skin emits infrared radiation mainly in the 2 - 20 micron wavelength range, with an average peak at 9-10 microns. Since the emissivity of human skin is extremely high (within 1\% of that of a black body), sensors in medical systems can measure infrared radiation emitted by the skin and convert it directly into precise temperature values using the Stefan-Boltzmann law. Each calculated temperature is encoded with a different color to generate a temperature map.

Thermographic assessments must take place in a controlled environment. The principal reason for this is the nature of human physiology. Changes from a different external environment, clothing, etc. can produce undesirable thermal effects. According 
to a report by Amalu (2001), abstaining from sun exposure, cosmetics and lotions before the procedure, along with 15 minutes of acclimation in a florescent lit, draft and sunlightfree, temperature and humidity-controlled room maintained between $18-22{ }^{\circ} \mathrm{C}$, and kept to within 1 degree of change during the procedure, is necessary to produce a physiologically neutral image free from interference.

\subsection{Modeling Heat Transfer in Tumors}

\subsubsection{Biology of Tumor Growth}

This section presents the biological basis of tumor growth that is later used in developing a model to get temperature distributions around tumors. The growth of tumors described here is based on the model proposed by Judah Folkman (1976). The growth usually starts with a single abnormal cell produced during the process of cell division. A single deviant cell capable of reproducing itself can rapidly develop into an in-situ lump that is characteristic of the earliest stage of a solid tumor. Cancer is more likely to develop in tissues in which cells divide rapidly. Breast tissue is especially sensitive to developing cancer since the female hormone estrogen promotes rapid division of breast cells, increasing the risk of cancer.

During the early stage of its development, the tumor is only a few millimeters in diameter and resembles a compressed sphere in shape. The tumor is called avascular since it has not yet developed its own vascular network of blood vessels for the supply of nutrients to its cells. The abnormal tumor cells require adequate supply of oxygen, amino acids and glucose in order to divide and reproduce rapidly. During the early stage of tumor development, these nutrients are secured by means of diffusion through surrounding healthy tissue. The removal of waste materials produced during cell division is also achieved through diffusion process. In case of a two dimensional tumor, the surface area and the volume of the tumor would increase at the same rate. However, since a tumor tends to grow in all dimensions, its shape more closely resembles that of a sphere. The diffusion process only supplies nutrients to the outer cells and may be 
incapable of nourishing the cells at the center. The tumor eventually reaches a steady state and may lie dormant for many years.

According to Folkman, a critical stage in the growth of a tumor is reached when it starts releasing a diffusible chemical substance called Tumor Angiogenesis Factor (TAF). The angiogenic factors released by the tumor diffuse into the surrounding tissue. The first reaction to this stimulus is that the endothelial cells in the neighboring vessels and closest to the chemical source start to modify their structure. After being stimulated by the TAF, the endothelial cells begin to migrate and accumulate at the site where the concentration of angeonic factors first reached a critical level. The vessel wall starts to swell as endothelial cells stack up to form sprouts. These capillary sprouts increase in size by gaining the endothelial cells from parent blood vessel. At a certain distance from the end of the sprout, endothelial cells begin to multiply. The sprouts are initially parallel but they grow towards each other as they elongate. Adjacent sprouts eventually fuse together at their tips to form loops (anastomoses). This is followed by the beginning of circulation of blood.

Thus, the release of TAF triggers the growth of new capillaries towards the tumor mass that eventually pierce through it. The growing network of blood vessels efficiently supplies nutrients and removes waste material. The rate of cell division is much higher in the vascular stage and the tumor grows at a fast pace. Unlike healthy cells that are exact copies of their parent cells, the tumor cell division produces variants that are much more destructive. These cells may spread to other parts of the body and set up new independent colonies.

\subsubsection{The Bioheat Equation}

The two main mechanisms of heat transfer in biological tissue are conduction due to gradient in temperatures inside the tissue, and the process of convection by the perfusing blood. Blood perfusion is defined as the blood volume flow exchange per unit volume of the tissue. One of the ways of measuring it is in units of milliliters of blood per second per milliliter of the tissue. While the flow of blood through arteries and veins is directional, blood perfusion is non-directional in nature and represents the dispersal of 
blood through capillaries and extracellular spaces of the tissue. The governing equation presented by Pennes is later used in developing the numerical model. The steady state Pennes bioheat equation describing the flow of thermal energy through a control volume of tissue is given by:

$$
k \nabla^{2} T \quad+\rho_{b} C_{b} \omega_{b}\left(T_{a}-T\right) \quad+\dot{Q}_{m}=0
$$

where $\mathrm{T}$ is the unknown temperature, $\mathrm{k}$ is the thermal conductivity, $\omega_{\mathrm{b}}$ is a term that represents blood perfusion, $C_{b}$ is the specific heat, $\rho_{b}$ is the density, $T_{a}$ is the arterial temperature, and $\mathrm{Q}_{\mathrm{m}}$ is volumetric metabolic heat generation rate inside the tissue. In the presence of a tumor, the metabolic heat generation rate $\mathrm{Q}_{\mathrm{m}}$ is significantly higher due to higher metabolic activity in the tissue. As discussed in the literature review, the tumor also has higher blood perfusion $\omega_{\mathrm{b}}$ due to the growing network of capillaries and blood vessels that it develops.

\subsection{Temperature Distributions due to a Tumor}

Lawson and Chughtai (1963) were among the first to note that a correlation exists between the temperature rise and the degree of malignancy of a tumor. Temperature measurements were taken by using the technique of direct thermistor thermometry during masectomy operations. Based on this study, they claimed that this difference in temperature cannot be accounted for on the basis of increased circulation. They further claimed that "vascular flow drains off the heat energy and thus actually cools the tumor in spite of serving the tumor's increased metabolic demands.”

Draper and Boag (1971) studied thermal patterns on the skin surface over veins

and tumors. They estimated the rate of heat generation within metabolizing tissue using the rate at which oxygen is consumed, or in case of anaerobic metabolism, the rate of formation of carbon dioxide. The rates of oxygen consumption in human tissue, for different parts of the body, were compiled from Roskelley et al. (1943), Albritton (1954), and Booth et al. (1967). The oxygen consumption in turn depends on the nutrients available to the tissue. Carcinoma cells consume nutrients and oxygen at a much higher 
rate compared to normal tissue. Based on these studies it was estimated that if all the energy available is released as heat, the maximum rate of heat production in a carcinoma is not likely to be greater than $25000 \mathrm{~W} / \mathrm{m}^{3}$. In order to study the thermal effect of tumors, they were treated as buried heat sources with a constant temperature of $37^{\circ} \mathrm{C}$. The resulting surface temperature profiles showed a wide and diffuse shape if the tumor was large and located deep below skin surface, but they show sharp peaks if the tumor considered was small and located closer to the skin surface. For several different heat generation rates in the tumor, the minimum size of the tumor necessary for any thermographic detection was calculated as a function of depth.

Nilsson et al. (1980) performed experimental studies and theoretical calculations to study surface temperature increases over an artificial heat source implanted in the human body. They found that the maximum increase in temperature on the skin surface above the embedded heat source is almost lineraly related to both the depth of the heat source and its power output. It was also concluded that a high power output or close proximity of the heat source to the skin surface is necessary in order to produce measurable temperature differentials.

Sudarshan et al. (1998) studied the temperature distributions in breast cancer by considering a two-dimensional model first proposed by Romrel et al. (1991). In this model, Pennes bioheat equation was used to describe the flow of thermal energy. The breast was modeled with various layers of different thicknesses. The model consists of a subcutaneous fat layer, followed by a gland layer and a deep muscle layer. The muscle layer lies adjacent to the thoracic wall. The thoracic wall is maintained close to the arterial temperature of $37{ }^{\circ} \mathrm{C}$ by the circulation of blood. The values of thermal conductivity, metabolic heat production and blood perfusion for each of these layers were taken from Werner and Buse (1988). The heat transfer coefficient h, due to the combined effect of convection, radiation and evaporation was adopted to be $13.5 \mathrm{~W} / \mathrm{m}^{2}{ }^{\circ} \mathrm{C}$ from Pennes (1948). Temperature profiles were generated by varying the tumor size, depth and the blood perfusion rate. The temperature profiles showed that the surface temperature reaches a maximum directly above the location of the tumor, and drops down as we move away on either side. This effect increases with increase in tumor size. Based on the study of different perfusion terms, it was concluded that the skin perfusion term has a greater 
effect on the maximum surface temperature than due to an increase in the gland perfusion term. It was also observed that the effect on the surface temperature is higher for low seated tumors than those that are deeply located. For a deep seated tumor and a given perfusion set, the maximum surface temperature is almost the same, regardless of the tumor size.

\subsection{Genetic Algorithms (GAs)}

\subsubsection{Background and Description}

Genetic Algorithms were first developed by John Holland (1975) at the University of Michigan. Holland's book titled "Adaptation in Natural and Artificial Systems”, published in 1975, is generally acknowledged as the beginning of the research

of genetic algorithms. Genetic algorithms borrow theories of evolutionary biology and mathematically apply them to optimization and parameter estimation problems. According to Yunker (1993), GAs were designed to "mimic the strategies that biologists have learned from evolution and apply them to optimization problems". GAs use techniques that are derived based on Darwin's principle of "survival of the fittest". This means that when a population of biological species evolves over time, characteristics that are beneficial to the survival of a species tend to be passed on to successive generations because the individuals carrying them get more opportunities to breed. Using GAs, the natural concepts of reproduction, crossover and mutation have been successfully applied to problems in search and optimization. According to Goldberg (1989), "GAs combine survival of the fittest among string structures with a structured yet randomized information exchange to form a search algorithm with some of the innovative flair of human search. In every generation, a new set of artificial creatures (strings) is created using bits and pieces of the fittest of the old; an occasional new part is tried for good measure." 


\subsubsection{Genetic Algorithms in Comparison to Traditional Methods}

GAs differ from traditional optimization and search procedures in four fundamental ways:

1. GAs work with a coding of the design variables, instead of the design variables themselves. The design variables are usually coded as finite-length strings.

2. In GAs, the search is from a population of design points, instead of jumping from one design point to another. This population of design points evolves from one generation to another. Fig. 2.1 shows the contour plot of function $F\left(x_{1}, x_{2}\right)$ in the design space.
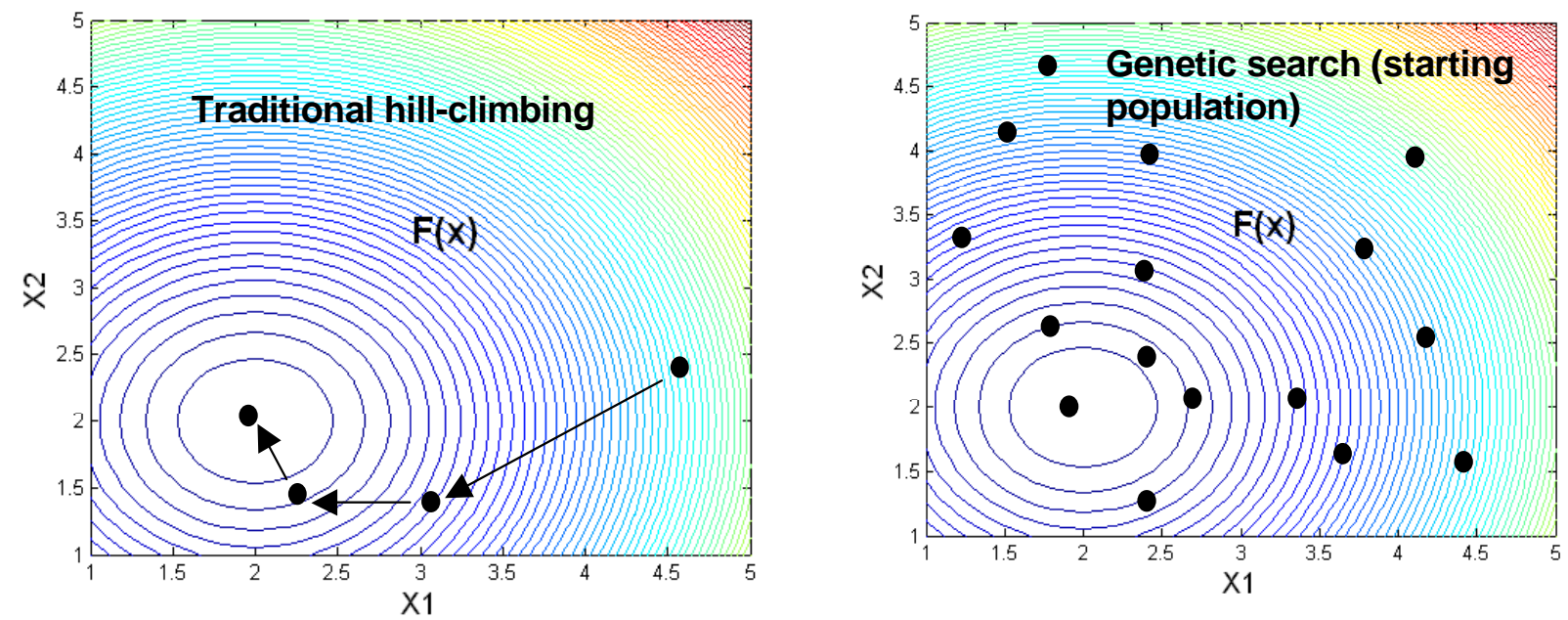

Fig. 2.1 A genetic search technique starts out with a population of initial points.

Here $\mathrm{x}_{1}$ and $\mathrm{x}_{2}$ are the design variables. When a genetic search is used to find the optimum, we start with a population of initial points instead of just one initial point. This makes GA searches more robust than traditional methods. According to Goldberg (1989), “the point-to-point method used in traditional optimization is dangerous because it is a perfect prescription for locating false peaks in multi-modal search spaces. By contrast, GAs work from a rich database of points simultaneously climbing many peaks in parallel. Thus, the probability of finding a false peak is reduced over methods that go point-by-point”. 
3. GAs work directly with function values. Unlike traditional methods, they do not need information about the derivative of the function. In cases where derivative information is hard to obtain, GAs can work fairly well.

4. Genetic reproduction is based on probabilistic rules and not on deterministic search schemes. GAs use random choices to guide a highly exploitative search.

\subsubsection{Recent Applications of Genetic Algorithms}

The robustness of GAs have resulted in their use in diverse fields such as optimization in engineering and computer science, structural optimization, automatic programming and machine learning, biotechnololgy, economics and social sciences, financial forecasting, art, music, and so on. The "Handbook of Genetic Algorithms" by Davis (1991) covers a wide range of applications of GAs in the real world. Some of the recent applications of GAs in the field of structural optimization, heat transfer and medical imaging are outlined below.

Goldberg and Samtani (1986) applied a GA to the optimization of a plane truss. The objective of the problem was to minimize the weight of the structure given the maximum and minimum stress constraints on members of the truss. The areas of the truss members were used as design variables and the GA consisted of roulette wheel selection, crossover and mutation operations. Furuya and Haftka (1993) used GAs with integer coding in order to find the optimal locations for the actuators on large space structures. Doyle (1995) applied GAs in order to find the size and location of a crack in a frame structure.

Rubinsky and Davalos (1998) were among the first to attempt to use a GA for solution of problems in the field of heat transfer, particularly the conduction heat transfer equation. They successfully applied GAs to solve the problem of a 2-D slab with fixed temperature boundary conditions. However, the algorithm worked well only with coarse mesh sizes (9 unknown nodes). The size of the search space of total possible solutions 
grew enormously with even a modest increase in the refinement of the mesh. Tsourkas and Rubinsky (2003) realized the source of these difficulties to be the np-hard nature of the problem and used a novel "genetic engineering" operator to resolve these difficulties. The novel "genetic engineering" operator is similar to gradient-like bitwise improvement technique mentioned by Goldberg (1989). Using this new algorithm, Tsourkas and Rubinsky were able to solve much finer meshes than was previously possible. The algorithm was tested with fixed temperature, convection and prescribed heat flux boundary conditions. Garcia (1999) applied GA for experimental design optimization and thermophysical parameter estimation of composite materials, where correlated parameters and low value of sensitivities make gradient-based methods unstable.

Fitzpatrick, Grefenstette and Van Gucht (1984) used GAs to perform medical image registration as a part of a larger digital subtraction angiography (DAS) system. DAS is used in order to examine the interior of a suspect artery by injection of a dye into the artery. Two x-ray images are taken, one before the injection of the dye, and one after the injection. The two images are converted to a digital format and subtracted pixel by pixel. The result is a subtracted image that shows the interior of the suspect artery. If the only disparity between the two x-ray images is the addition of the dye, then the difference image shows only the dye-coated regions. Since movement of the patient during the x-ray procedure can produce images that are misaligned, alignment or registration of the two images is necessary prior to the subtraction procedure. Fitzpatrick et al. used a bilinear mapping formula with unknown coefficients to transform the pre-injection image. A GA was then implemented to search for the unknown coefficients that minimized the difference between pre-injection and post-injection images using the mean absolute image difference as the criterion. Numerical experiments with both simulated images and real x-ray images were successful.

From the applications described above, it can be seen that GAs can be used as robust non-gradient based optimization procedures. Therefore, in this research, these algorithms were implemented to determine the location and heat generation rate of an embedded tumor, using a binary encoding for these parameters. 


\section{Chapter 3 Numerical Model and Estimation Methodology}

\section{$3.1 \quad$ Numerical Model}

Due to uncertainty in the shape and location of an embedded tumor acting as a heat source, exact analytic solutions become impossible to apply and numerical solutions are employed instead. The numerical finite difference model used to predict the surface temperature distribution is also called the direct model and is based on the bioheat equation that was given in Equation (2.2). In this study, a simplified model was used, as shown in Fig. 3.1. The metabolism in a tumor is represented as a planar heat source in this model to be compatible with the experimental validation described later in Chapter 5. The model is highly flexible since it can be modified to be used with any combination of boundary conditions, and has the ability to include both internal heat generation of the tissue and blood perfusion. For example, in case of breast cancer, the cylinder can be used to represent the breast, with lower boundary maintained at core body temperature of $37^{\circ} \mathrm{C}$, while all other boundaries exposed to convection.

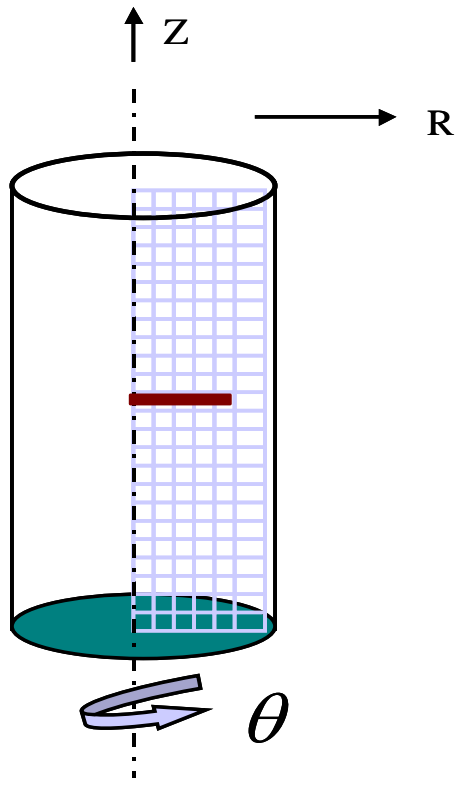

Fig. 3.1 Finite difference scheme in cylindrical coordinates 
The numerical model was employed with both simulated and experimental data in order to test the validity of this model. The model has the radius and height of the cylinder to be $0.073 \mathrm{~m}$ and $0.0635 \mathrm{~m}$ respectively. The shaded area in Fig. 3.1 has a radius of $0.023 \mathrm{~m}$ and represents the region of the tumor that has different thermal properties than the rest of the domain representing healthy tissue. The cylinder was considered insulated on all sides except the top surface. The top surface of the cylinder was exposed to convection. The results of the simulation and experiments are presented in Chapter 6. Although the model developed here is in a cylindrical coordinate system, it can easily be modified for use in other coordinate systems.

\subsubsection{Finite Difference Scheme}

The finite difference method employed here has been developed by using the approach taken by Patankar (1980) and Vick (2003). It is based on the two-dimensional steady state heat equation in cylindrical coordinates that is given by:

$$
\frac{1}{r} \frac{\partial}{\partial r}\left(k r \frac{\partial T}{\partial r}\right)+\frac{\partial}{\partial z}\left(k \frac{\partial T}{\partial z}\right)+S(r, z, T)=0
$$

where $\mathrm{T}$ is the unknown temperature, $\mathrm{k}$ is the thermal conductivity, and $\mathrm{S}(\mathrm{r}, \mathrm{z}, \mathrm{T})$ is the source term. According to Vick (2003), the source term provides a lot of flexibility for representation of the physical problem. It can incorporate both the heat generation term and the fin term. The source term is further broken down into a temperature independent term $S_{c}(r, z)$ and a temperature dependent term $S_{p}(r, z, T)$. Equation (3.1) can now be rewritten as:

$$
\frac{1}{r} \frac{\partial}{\partial r}\left(k r \frac{\partial T}{\partial r}\right)+\frac{\partial}{\partial z}\left(k \frac{\partial T}{\partial z}\right)+S_{C}(r, z)-S_{P}(r, z, T) \quad T=0
$$

Equation (3.2) has the same form as the steady state Pennes bioheat equation given by Equation (2.2). Therefore, it was concluded that a discretization scheme based on 
Equation (3.2) would be well suited for modeling the flow of thermal energy through a control volume of tissue. The $\mathrm{S}_{\mathrm{c}}(\mathrm{r}, \mathrm{z})$ term can be used to specify the internal heat generation rate within the tissue, while the $\mathrm{S}_{\mathrm{p}}(\mathrm{r}, \mathrm{z}, \mathrm{T})$ term gives the option of incorporating blood perfusion in the model. The two-dimensional domain is discretized as shown in Fig. 3.2. The domain is completely filled with control volumes and the gird points at the four boundaries are surrounded by imaginary control volumes that have zero thickness. The advantage of using this scheme is that boundary conditions such as given temperature, heat flux and convection can be incorporated directly into the finite difference equations.

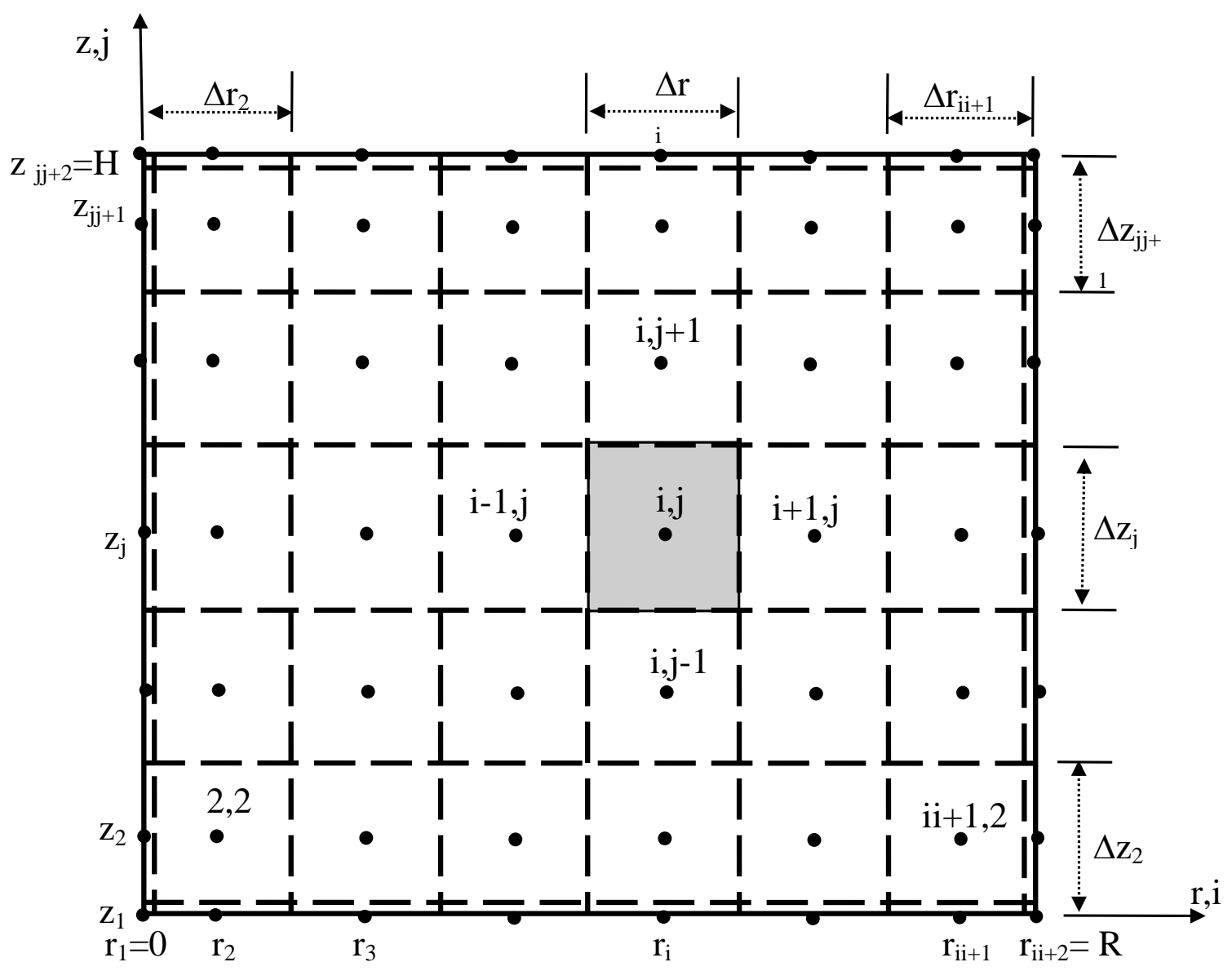

Fig. 3.2 Discretization of two-dimensional domain in cylindrical coordinates (Vick, 2003) 
A typical control volume in the domain surrounded by its east, west, north and south neighbors is shown in Fig. 3.3.

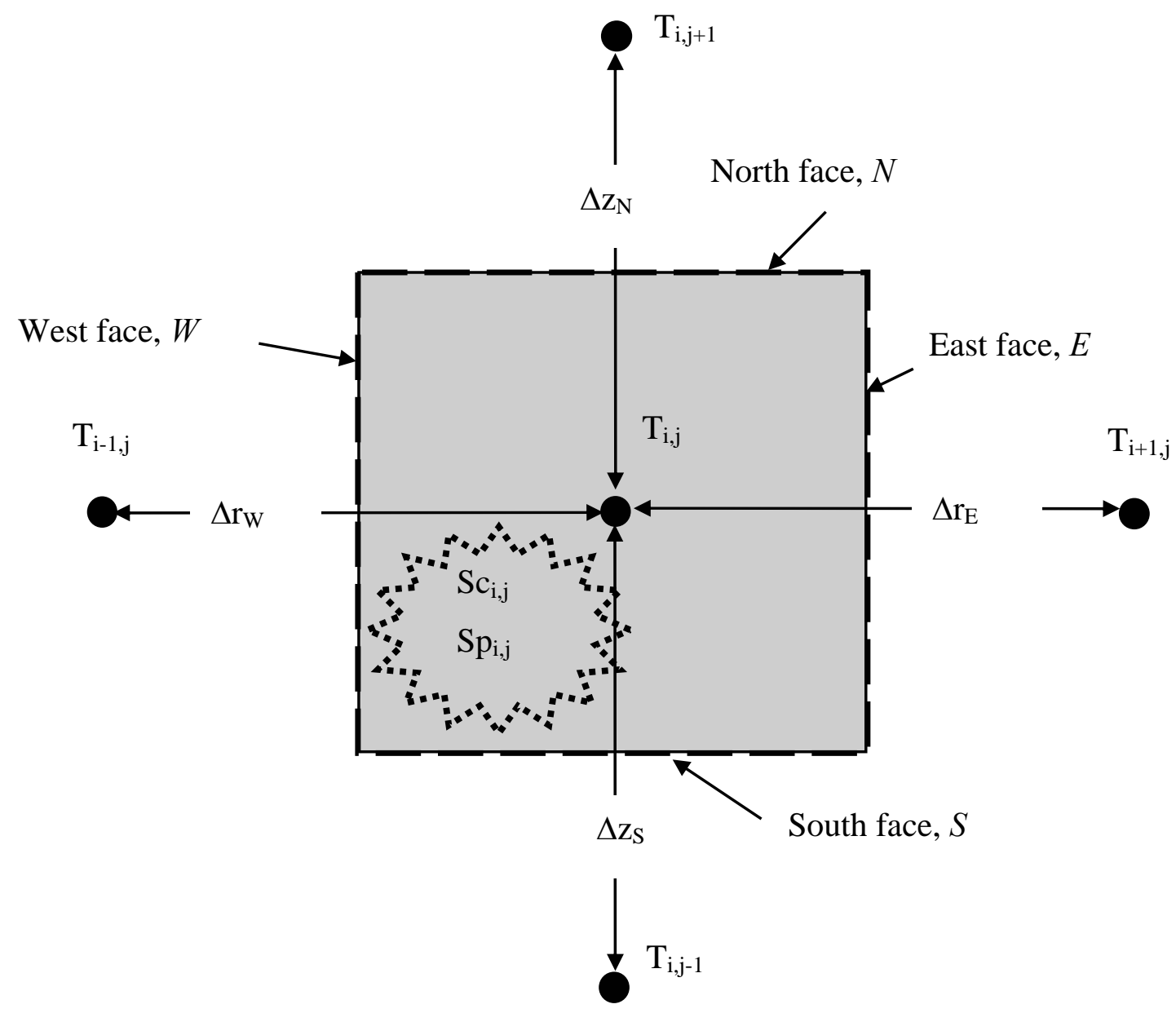

Fig. 3.3 A control volume in the two-dimensional domain (Vick, 2003)

Equation (3.2) is integrated over the area of control volume (i, j) shown in Fig. 3.3.

$\int_{S}^{N} \int_{W}^{E}\left[\frac{1}{r} \frac{\partial}{\partial r}\left(k r \frac{\partial T}{\partial r}\right)+\frac{\partial}{\partial z}\left(k \frac{\partial T}{\partial z}\right)+S_{C}(r, z)-S_{P}(r, z, T) \quad T\right] r d r d z=0$ 
Equation (3.4) is integrated both in the radial and vertical direction. The temperature gradient terms are evaluated using piecewise linear profiles between the grid points. We get an equation of the form:

$$
\begin{aligned}
& k_{E}\left(\frac{T_{i+1, j}-T_{i, j}}{\Delta r_{E}}\right) \Delta z_{j}-k_{W}\left(\frac{T_{i, j}-T_{i-1, j}}{\Delta r_{W}}\right) \Delta z_{j}+k_{N}\left(\frac{T_{i, j+1}-T_{i, j}}{\Delta z_{N}}\right) r_{i, j} \Delta r_{i} \\
& -k_{S}\left(\frac{T_{i, j}-T_{i, j-1}}{\Delta z_{S}}\right) r_{i, j} \Delta r_{i}+S c_{i, j} r_{i, j} \Delta r_{i, j} \Delta z_{j}-S p_{i, j} T_{i, j} r_{i, j} \Delta r_{i, j} \Delta z_{j}=0
\end{aligned}
$$

Here $S c_{i}$ and $S p_{i}$ represent average values of $S c$ and $S p$ in the control volume. The interface conductivity at the east, west, north and south faces of the control volume $(i, j)$ is represented by $\mathrm{k}_{\mathrm{E}}, \mathrm{k}_{\mathrm{W}}, \mathrm{k}_{\mathrm{N}}$ and $\mathrm{k}_{\mathrm{S}}$ respectively. It is useful to cast Equation (3.5) into the following form:

$a_{i, j} T_{i, j}-a E_{i, j} T_{i+1, j}-a W_{i, j} T_{i-1, j}-a N_{i, j} T_{i, j+1}-a S_{i, j} T_{i, j-1}=b_{i, j}$

where

$$
\begin{aligned}
& a E_{i, j}=\frac{k_{E}}{\Delta r_{E}} \Delta z_{j}=\left(\frac{2 k_{i, j} k_{i+1, j}}{\Delta r_{i} k_{i+1, j}+\Delta r_{i+1} k_{i, j}}\right) \Delta z_{j} \\
& a W_{i, j}=\frac{k_{W}}{\Delta r_{W}} \Delta z_{j}=\left(\frac{2 k_{i-1, j} k_{i, j}}{\Delta r_{i-1} k_{i, j}+\Delta r_{i} k_{i-1, j}}\right) \Delta z_{j} \\
& a N_{i, j}=\frac{k_{N}}{\Delta z_{N}} r_{i, j} \Delta r_{i}=\left(\frac{2 k_{i, j} k_{i, j+1}}{\Delta z_{j} k_{i, j+1}+\Delta z_{j+1} k_{i, j}}\right) r_{i, j} \Delta r_{i} \\
& a S_{i}=\frac{k_{S}}{\Delta z_{S}} r_{i, j} \Delta r_{i}=\left(\frac{2 k_{i, j-1} k_{i, j}}{\Delta y_{j-1} k_{i, j}+\Delta y_{j} k_{i, j-1}}\right) r_{i, j} \Delta r_{i}
\end{aligned}
$$


$a_{i, j}=a E_{i, j}+a W_{i, j}+a N_{i, j}+a S_{i, j}+S p_{i, j} r_{i, j} \Delta r_{i, j} \Delta z_{j}$

$b_{i, j}=S c_{i, j} r_{i, j} \Delta r_{i, j} \Delta z_{j}$

In general, Equation (3.6) can be thought of as having the form:

$$
a_{P} T_{P}-\sum a_{n b} T_{n b}=b
$$

where $T_{P}$ represents the temperature of the central grid point, subscript nb denotes a neighbor, and the summation is taken over all neighboring cells.

The formulation given by Equation (3.6) is valid for internal control volumes of the domain, ranging from control volume 2 to $\mathrm{ii}+1$. The zero control volumes at the boundaries are used to incorporate the boundary conditions that can be specified as some combination of heat flux and convective conditions. For example, the boundary condition at the outer surface of the cylinder, for some specified heat flux $\mathrm{q}_{\mathrm{R}}$ " and convective conditions $T_{\infty}$ and $h_{R}$, can be written as:

$-k \frac{\partial T}{\partial r}=q_{R}^{\prime \prime}+h_{R}\left(T_{\infty}-T\right)$

The discretized form of Equation (3.9), expressed in terms of coefficients is:

$a_{i i+2, j} T_{i i+2, j}-a W_{i i+2, j} T_{i i+1, j}=b_{i i+2, j}$

where

$$
\begin{aligned}
& a W_{i i+2, j}=\frac{k_{i i+1, j}}{\Delta r_{i i+1, j} / 2} \\
& a_{i i+2, j}=a W_{i i+2, j}+h_{R} \\
& b_{i i+2, j}=q_{R}^{\prime \prime}+h_{R} T_{\infty}
\end{aligned}
$$


If the boundary condition is a specified temperature $T_{R}$, then Equation (3.10) can be used with the following coefficients:

$\mathrm{aW}_{\mathrm{ii}+2, \mathrm{j}}=0, \mathrm{a}_{\mathrm{ii}+2, \mathrm{j}}=1, \mathrm{~b}_{\mathrm{ii}+2, \mathrm{j}}=\mathrm{T}_{\mathrm{R}}$

The boundary conditions at $\mathrm{r}=0, \mathrm{z}=0$ and $\mathrm{z}=\mathrm{H}$ can be derived in a similar manner.

\section{Solution of the Algebraic Equations}

The solution of the algebraic equations is carried out using a method known as ADI (Alternating-Direction Implicit) that was introduced by Peaceman and Rachford (1955). In the ADI method, the discretized equations for the grid points along a line in the chosen direction are considered. The temperatures of the two neighboring lines are considered to be known and equal to their last updated values. The discretized equations can then be assembled in the form of a tridiagonal matrix where all elements are zero except the diagonal and the elements to the immediate left and right of the diagonal. The temperatures along the chosen line can then be solved easily using the Tridiagonal Matrix Algorithm Method (TDMA), also known as the Thomas Algorithm. This procedure is carried out for all lines in the radial direction, and then all lines in the vertical direction. By alternating the directions in which the TDMA is used, the rate of convergence is accelerated because the information from the boundary reaches the interior quickly. A representation of the ADI method is shown in Fig. 3.4. 


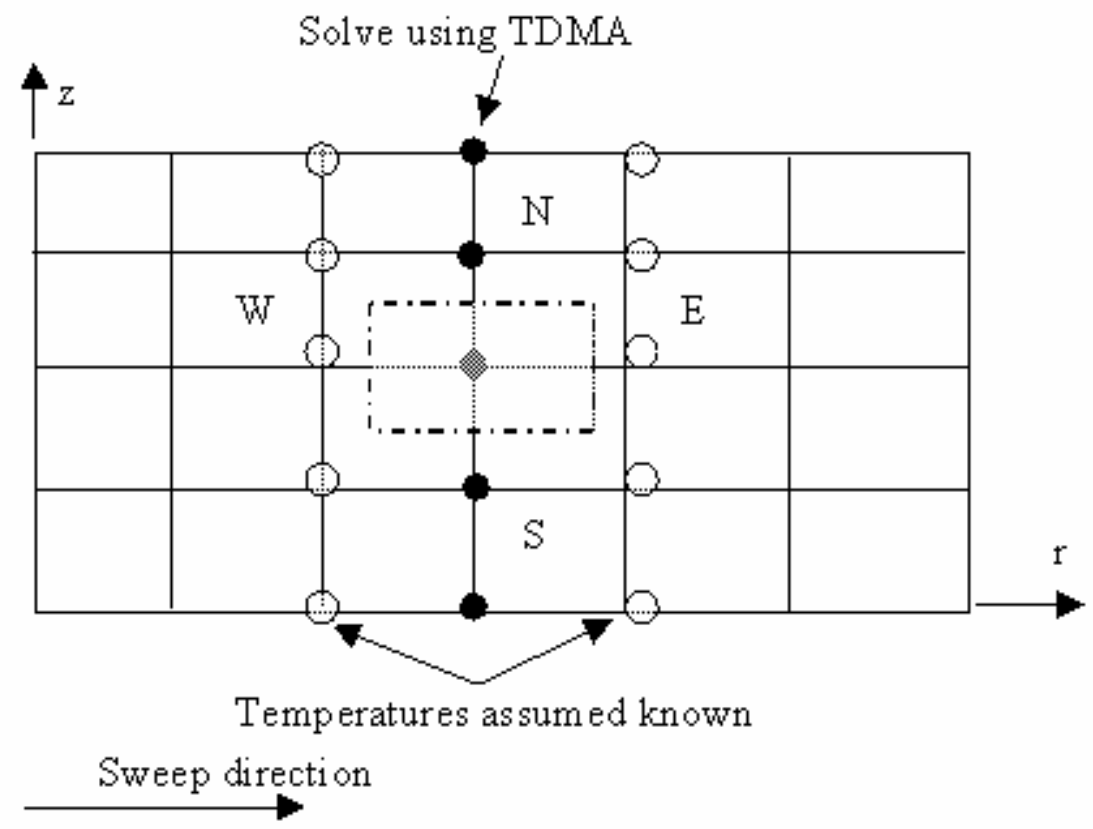

Fig. 3.4 Representation of the ADI method in the two dimensional domain

Computer codes that implement the finite difference method, the ADI method and the TDMA are given in Appendix A,B and $\mathrm{C}$ respectively.

\subsection{Estimation Methodology}

The parameter estimation method used in this study requires both the computed and the experimentally determined temperature data. First, the objective function is described. Then, some gradient-based methods for evaluating the objective function are presented. These are followed by a sensitivity analysis of the problem in order to show the shortcomings of the gradient-based estimation methods. Finally, a genetic algorithm is presented as the parameter estimation method used for this problem. A flowchart showing the estimation procedure is shown in Fig. 3.5. 


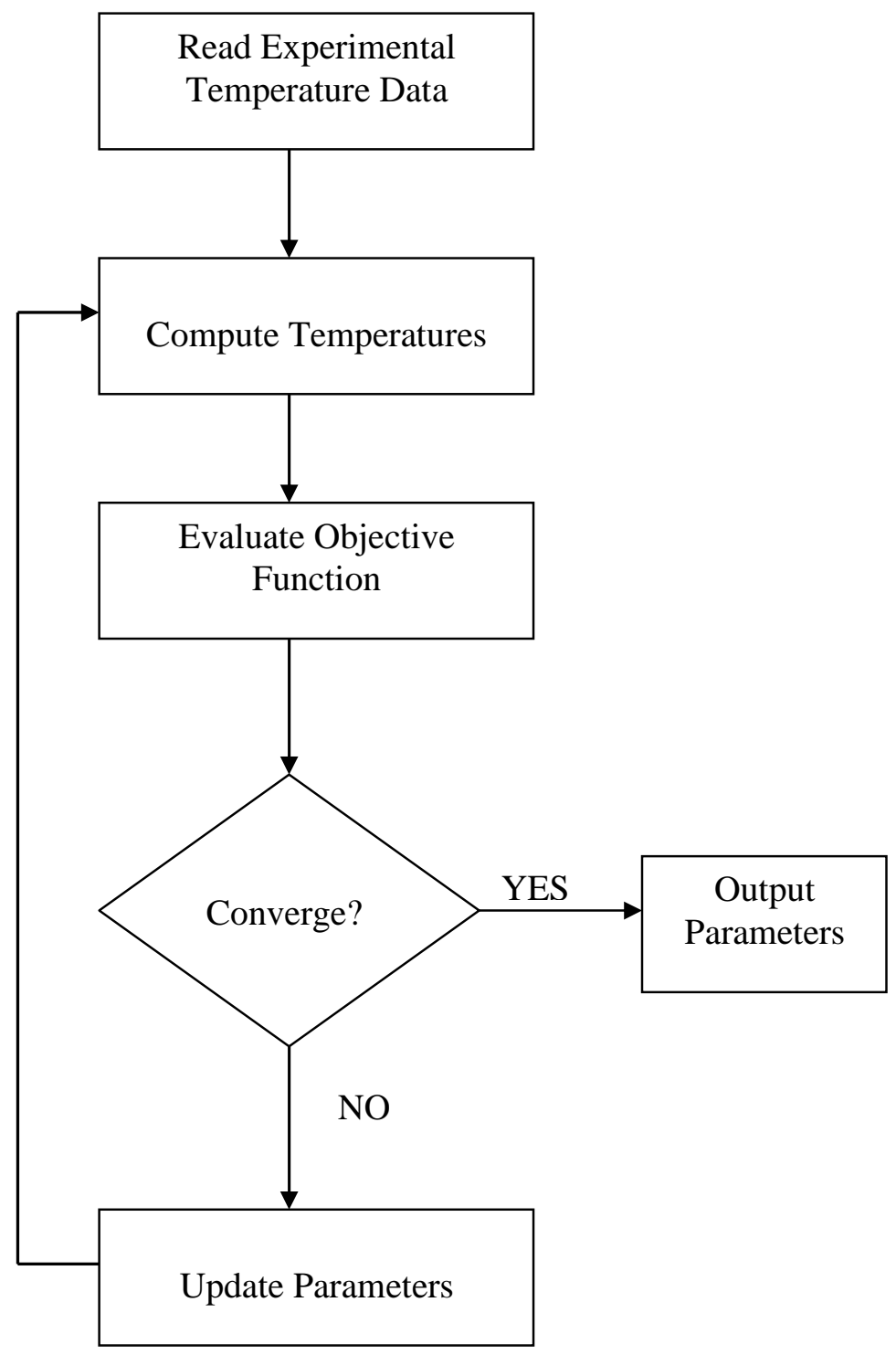

Fig. 3.5 A flowchart of the estimation program

\subsubsection{The Objective Function}

The objective function used here is a sum of the squares function that relates the data from the model to the corresponding experimental data. It measures the distance between the estimated and the measured skin surface temperatures. The sum of the squares is defined as:

$$
S=[\mathbf{Y}-\mathbf{T}(\beta)]^{T}[\mathbf{Y}-\mathbf{T}(\beta)]
$$


where $\mathbf{Y}$ and $\mathbf{T}$ are vectors containing the measured and estimated temperatures, respectively, and subscript $\mathrm{T}$ denotes the transpose of the vector. The estimated temperatures are obtained by the solution of the direct problem using estimates for the unknown parameters. The vector containing the estimates for unknown parameters is denoted by $\beta$. In this study, the unknown parameter vector $\beta$ contains the depth and the heat generation rate of the tumor. A plot of sum of squares function for a tumor located $0.035 \mathrm{~m}$ from the base and having heat generation rate of $1400 \mathrm{~W} / \mathrm{m}^{3}$, is shown is Fig. 3.6.

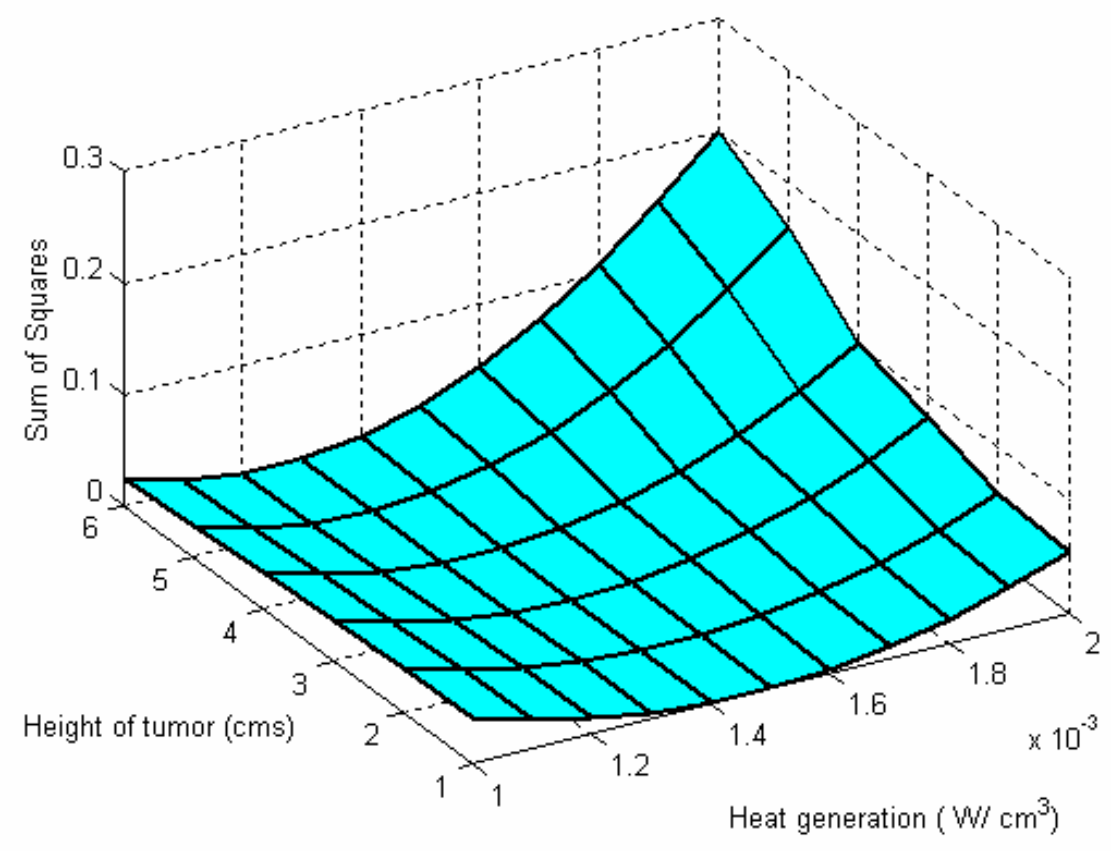

Fig. 3.6 The variation of sum of squares function with tumor height and heat generation rate

\subsubsection{Gradient Based Parameter Estimation Approach}

The most commonly used parameter estimation techniques in heat transfer analysis are Gauss minimization and Box-Kanemasu Interpolation method. These techniques are described below. 


\section{The Gauss Minimization Method}

Gauss minimization is a gradient based method that minimizes the objective function by setting its derivate with respect to the unknown parameters equal to zero. Gauss minimization is an iterative method that specifies both the direction and magnitude of corrections to the parameters, until the parameters that minimize the objective function are found. This method is effective in seeking minima if the initial estimates are in the general region of the minimum (Beck and Arnold , 1972).

A necessary condition at the minimum value of objective function $S$ is that the derivative of $S$ with respect to the parameter vector $\beta$ must be equal to zero:

$$
\nabla_{\beta} S=2\left[-\nabla_{\beta} \mathbf{T}^{T}(\beta)\right][\mathbf{Y}-\mathbf{T}(\beta)]=0
$$

The first term on the right hand side of equation (3.13) is the derivative of the estimated temperature vector $\mathbf{T}$ with respect to the parameter vector $\beta$. The transpose of this matrix is called the sensitivity matrix.

$$
\mathbf{X}(\beta) \equiv\left[-\nabla_{\beta} \mathbf{T}^{T}(\beta)\right]^{T}
$$

Without showing the dependence of $\mathbf{X}$ on parameter vector $\beta$, the sensitivity matrix can be written as:

$$
\mathbf{X}=\left[\begin{array}{ccccc}
\frac{\partial \hat{T}_{1}}{\partial \beta_{1}} & \frac{\partial \hat{T}_{1}}{\partial \beta_{2}} & \cdot & \cdot & \frac{\partial \hat{T}_{1}}{\partial \beta_{n}} \\
\frac{\partial \hat{T}_{2}}{\partial \beta_{1}} & \frac{\partial \hat{T}_{2}}{\partial \beta_{2}} & \cdot & \cdot & \frac{\partial \hat{T_{2}}}{\partial \beta_{n}} \\
\cdot & \cdot & \cdot & \cdot & \cdot \\
\cdot \hat{T_{m}} & \frac{\partial \hat{T}_{m}}{\partial \beta_{1}} & \cdot & \cdot & \frac{\partial \hat{T}_{m}}{\partial \beta_{n}}
\end{array}\right]
$$


Each element of the sensitivity matrix $\mathbf{X}(\beta)$ is called a sensitivity coefficient, denoted by $\mathrm{X}_{\mathrm{ij}}$. For this case, a sensitivity coefficient is defined as the first derivative of an unknown temperature $T_{\mathrm{i}}$, with respect to a parameter $\beta_{\mathrm{j}}$.

$$
X_{i j}=\frac{\partial T_{i}}{\partial \beta_{j}}
$$

Two simplifying assumptions are used in the Gauss method. The matrix $\mathbf{X}(\beta)$ is replaced by $\mathbf{X}(\mathbf{b})$, where $\mathbf{b}$ is an approximation for $\beta$. The temperature vector $\mathbf{T}(\beta)$ is approximated by the first two terms of the Taylor series of $\mathbf{T}(\beta)$ about $\mathbf{b}$. Using these two assumptions, Equation (3.13) can be written as:

$$
\mathbf{X}^{T}(\mathbf{b})[\mathbf{Y}-\mathbf{T}(\mathbf{b})-\mathbf{X}(\mathbf{b})(\boldsymbol{\beta}-\mathbf{b})]=0
$$

Equation (3.17) is solved iteratively to find the parameter vector $\mathbf{b}^{(\mathrm{k}+1)}$ that minimizes the sum of squares objective function. The symbol $\mathrm{k}$ denotes the index of iterations.

$$
\begin{aligned}
& \mathbf{b}^{(k+1)}=\mathbf{b}^{(k)}+\Delta_{g} \mathbf{b}^{(k)} \\
& \Delta_{g} \mathbf{b}^{(k)}=\mathbf{P}^{(k)}\left[\mathbf{X}^{T(k)} \quad\left(\mathbf{Y}-\mathbf{T}^{k}\right)\right. \\
& \mathbf{P}^{-1(k)}=\mathbf{X}^{T(k)} \quad \mathbf{X}^{(k)}
\end{aligned}
$$

\section{Box-Kanemasu Estimation Method}

A modification to the Gauss method was proposed by Box and Kanemasu (1972) that uses the direction given by the Gauss method but modifies the step-size. The BoxKanemasu method was modified by Bard (1974) to incorporate a check that the sum of squares $\mathrm{S}$ decreases from one iteration to another. This method is called the modified Box-Kanemasu estimation method. 
The step-size used in the Gauss method is modified by the introduction of a scalar interpolation factor $\mathrm{h}^{(\mathrm{k}+1)}$. The index $(\mathrm{k}+1)$ denotes that $\mathrm{h}$ may be iteration dependent. Equation (3.18a) can be re-written as:

$$
\mathbf{b}^{(k+1)}=\mathbf{b}^{(k)}+h^{(k+1)} \Delta_{g} \mathbf{b}^{(k)}
$$

The scalar interpolation factor $\mathrm{h}^{(\mathrm{k}+1)}$ is calculated as:

$$
h^{(k+1)}=G^{(k)} \alpha^{2}\left[S_{\alpha}^{k}-S_{0}^{k}+2 G^{(k)} \alpha\right]^{-1}
$$

and

$$
G^{(k)}=\left[\Delta_{g} \mathbf{b}^{(k)}\right]^{T} \mathbf{P}^{-1(k)} \Delta_{g} \mathbf{b}^{(k)}
$$

In the Gauss method, the scalar interpolation factor given by Equation (3.19b) is set equal to 1. A flow chart of the Box-Kanemasu method is presented in Appendix D. A computer code to implement Box-Kanemasu method is given in Appendix E.

\subsubsection{Sensitivity Coefficients}

The sensitivity coefficients due to a parameter $\beta_{\mathrm{j}}$ can be written in dimensionless form as:

$$
X_{i j}=\frac{\partial T_{i}}{\partial \beta_{j}} \times \frac{\beta_{j}}{\Delta T_{M A X}}
$$

where $\Delta T_{M A X}$ is the maximum temperature difference on the surface of the medium. The term $\partial T_{i} / \partial \beta_{j}$ represents the change in temperature at a location $i$ on the surface due to a small change in the parameter $\beta_{\mathrm{j}}$. For the problem at hand, the parameters considered for sensitivity analysis are the radius and the depth of the tumor below the skin surface. The plot of dimensionless sensitivity coefficients due to radius and depth are shown in Fig. 3.7 and Fig. 3.8 respectively. These are sensitivity coefficients at a point on the surface that lies directly above the location of the embedded tumor. 


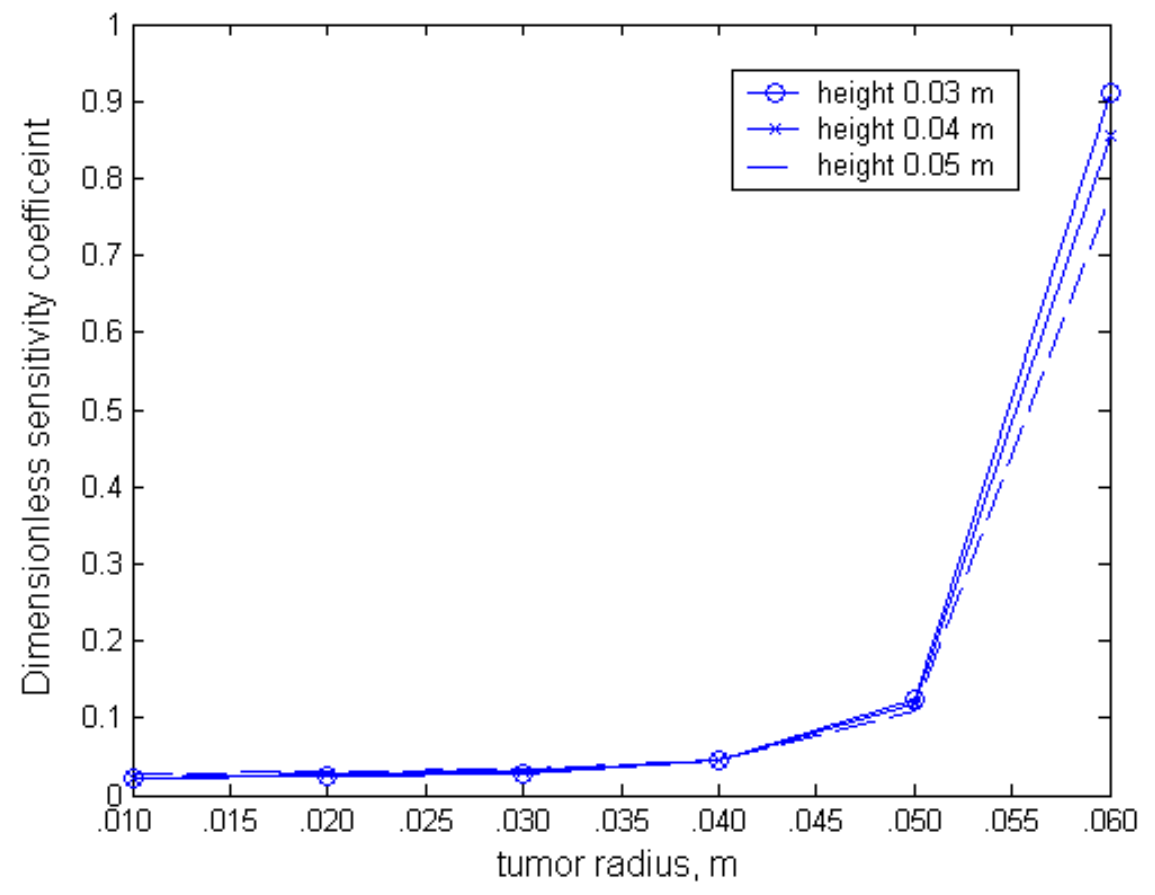

Fig. 3.7 Dimensionless sensitivity of temperature to tumor size for different tumor locations

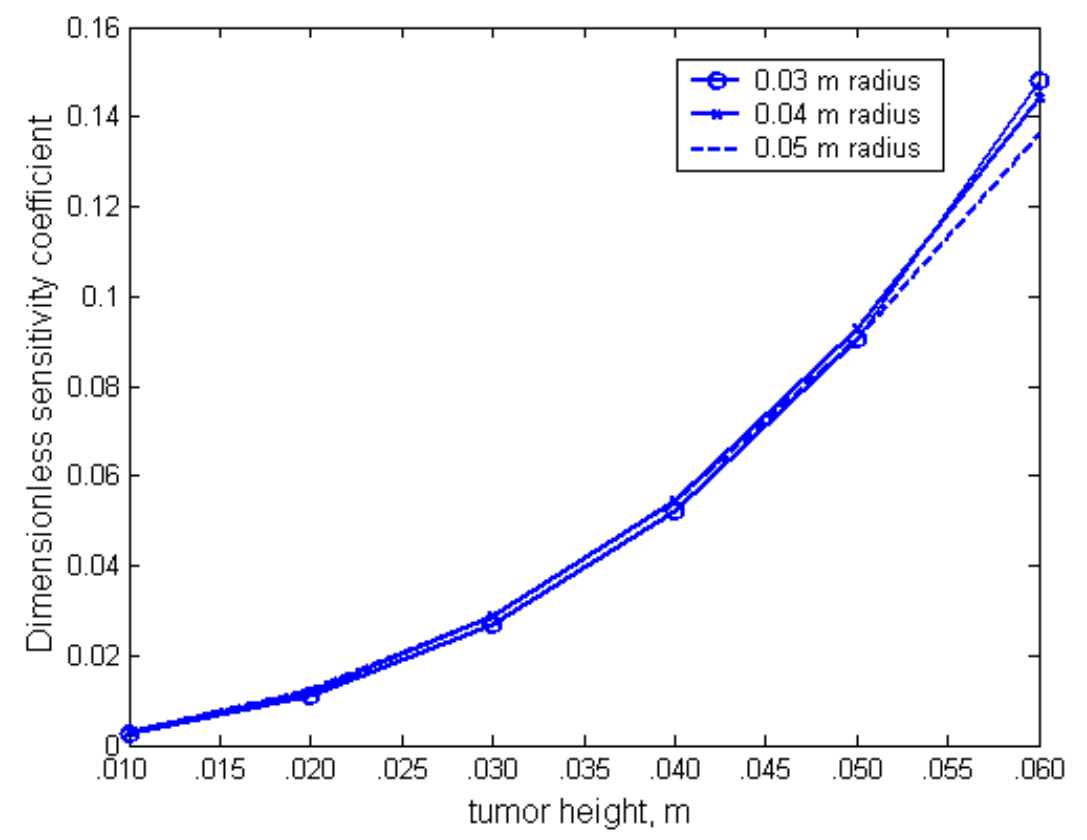

Fig. 3.8 Dimensionless sensitivity of temperature to tumor location for different tumor sizes 
Fig. 3.7 show that the sensitivity of the surface temperature to the radius of the tumor, is independent of the location. Similarly, Fig. 3.8 shows that the sensitivity of surface temperature to the depth of the tumor is independent of its size. The sensitivity coefficients can provide valuable insights into the estimation problem. If the sensitivity coefficients have a small magnitude, or if the sensitivity coefficients due to different parameters exhibit a correlation with one another, then the estimation problem becomes very difficult to solve. In order to examine the correlation of temperature sensitivity to tumor size and location, the dimensionless sensitivity coefficient curves due to these two parameters are shown superimposed in Fig. 3.9.

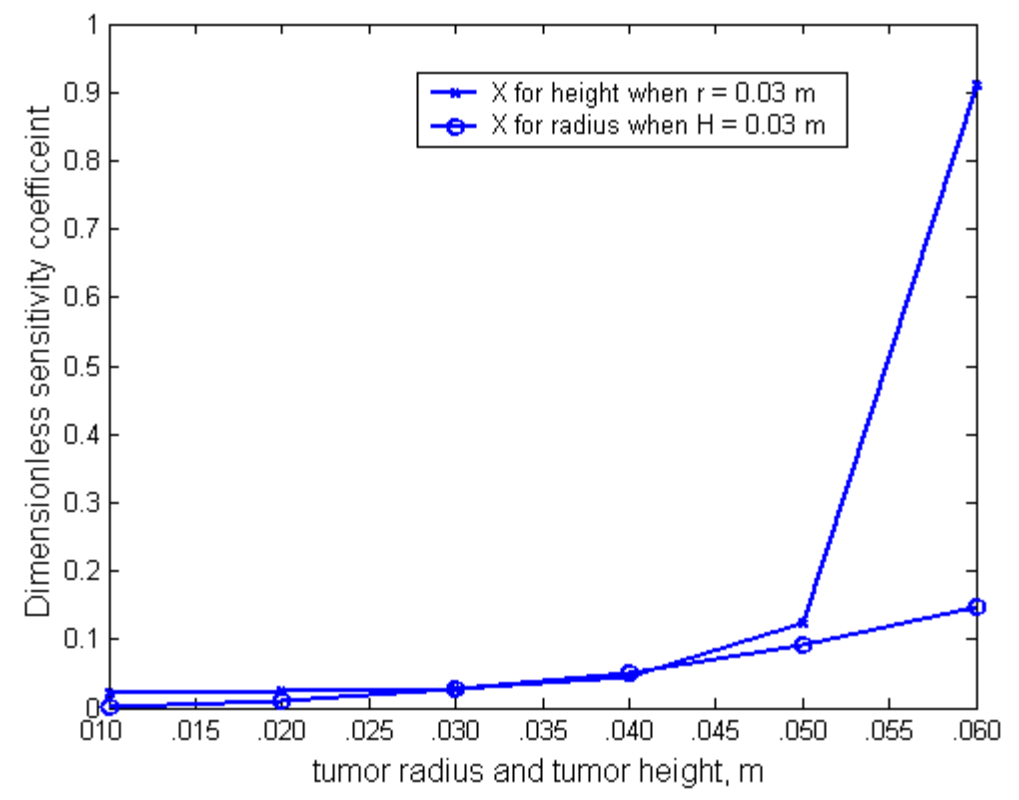

Fig. 3.9 Dimensionless sensitivity of temperature to tumor location and radius

From Fig. 3.9 it can be seen that the sensitivity coefficients due to tumor radius and depth have a small magnitude. This makes the use of gradient-based estimation techniques difficult and ineffective. Therefore, a non-gradient based approach was chosen as the parameter estimation method for this problem. It can also be seen from Fig. 3.9 that the sensitivity coefficients exhibit strong linear dependence on one another if the tumor is small in radius or if it is located below a certain depth. Due to this strong linear dependence, it becomes very difficult to estimate both the size and location of the tumor simultaneously. As mentioned earlier in Section 3.1, these results were derived by 
representing a three dimensional tumor as a planar heat source. Slightly different results can be expected if the heat source is spherical.

\subsubsection{Parameter Estimation Using Genetic Algorithms}

As noted in the last section, due to the drawbacks of the traditional estimation methods, a non-gradient based estimation approach was needed for solving the problem. GAs are robust and reliable and they do not suffer from difficulties encountered by traditional methods. GAs were therefore chosen to implement the estimation procedure. A detailed description of these algorithms is presented in the next chapter.

\subsubsection{Confidence Intervals}

The confidence intervals give us an indication of how far an estimate may be expected to be from the true value. The confidence interval can be thought of as a probability region for the mean value. A 95 percent confidence interval around a temperature estimate can be calculated by using the standard Student's-t formula:

$$
C I=\bar{T} \pm t(0.025, n-1) \frac{s}{\sqrt{n}}
$$

where $\bar{T}$ is the average measured temperature at a given location, $\mathrm{s}$ is the standard deviation around the average, and $\mathrm{n}$ is the number of observations in the sample. 


\section{Chapter 4 Genetic Algorithms (GAs)}

This chapter discusses genetic algorithms, the non-gradient based method selected to estimate the size and heat generation rate of the tumor. The algorithm is suited to the linear difficulties illustrated by the sensitivity analysis of the problem. A flowchart showing the procedure of the GAs is shown in Fig. 4.1.

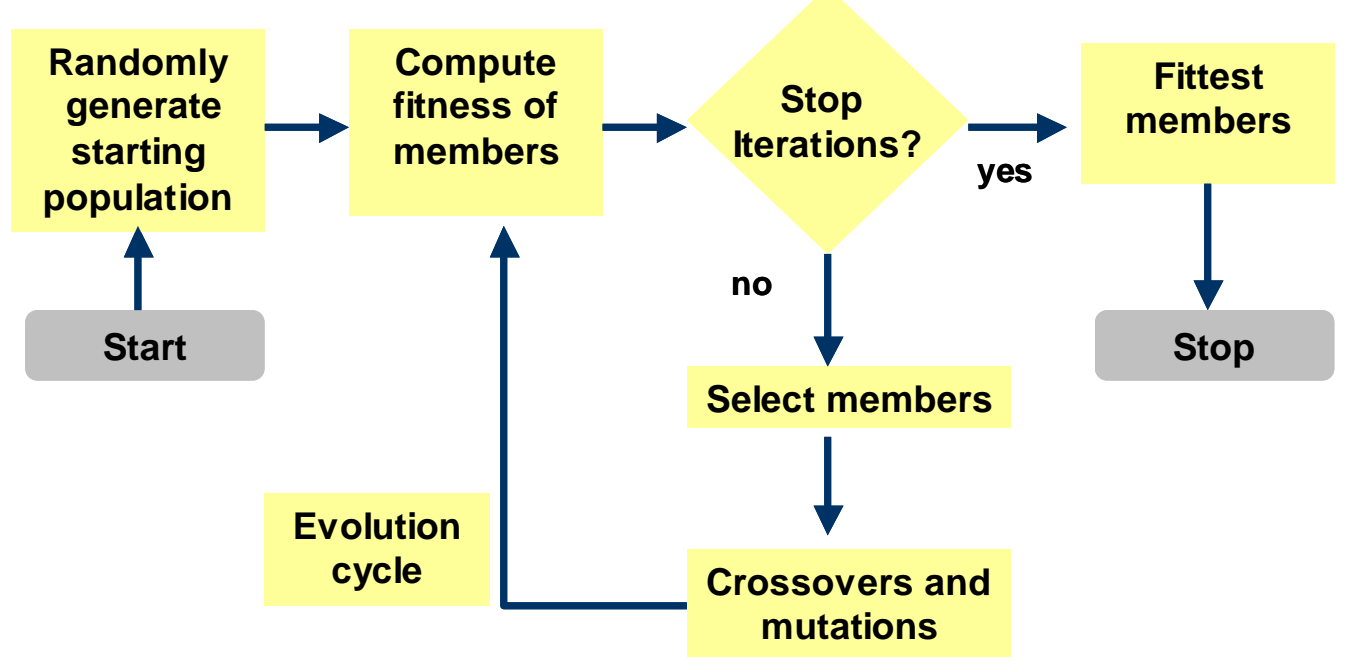

Fig. 4.1 Iterative procedure of a genetic algorithm

\subsection{Description}

Genetic algorithms function by generating an initial population of individuals or potential solutions to the optimization problem. This initial population reproduces and creates "children" or the next population. This new generation of individuals is evaluated for fitness. The fittest individuals (those that best minimize the objective function) will be selected to reproduce, passing their traits on to the next generation. The cycle is 
complete when subsequent populations cease to change or after a fixed number of iterations. Ideally, this is when the optimum solution has been found.

\subsubsection{Encoding}

Genetic algorithms are organized in the same manner as the genetic material of a cell. Each individual's cell contains chromosomes that dictate physical and psychological traits. Chromosomes, in turn, are constructed of genes. A gene encodes a specific feature of the individual. For example, a person's eye color is dictated by a specific gene. The actual value of the gene is called an allele. So the eye color gene may produce brown eyes. In GAs, a chromosome is an array of genes, and a gene is an array of data.

\section{Gene1 Gene2 Gene3 Gene4}

(11000010, 00001110, 001111010, 10100011)

The optimization algorithm uses this chromosome-gene structure to code the design parameters of the problem. The parameter of interest are converted to a binary string of numbers that can be modified by the code and optimized. Multiple parameters are coded into a single string.

\subsubsection{Selection}

Natural selection occurs according to what individuals are best suited to reproduce. For the genetic algorithm, this criterion is known as 'fitness'. Fitness relates how each chromosome of parameters satisfies the objective function. The closer the parameters come to fulfilling the objective function, the higher would be the numerical fitness assigned to that parameter. All these fitness values are added together to determine the total fitness of the group. The fitness of each chromosome can be viewed as a percentage of the total group fitness. This concept can be thought of as a pie chart with different sized wedges. Bigger wedges are chromosomes that have higher fitness.

The pie chart then becomes a roulette wheel where, at random, different chromosomes are selected to be parents to the next generation. Fitter individuals have a greater chance of selection, though less fit individuals may still be picked. Fig. 4.2 illustrates the roulette wheel method of selection. 
population

(binary)

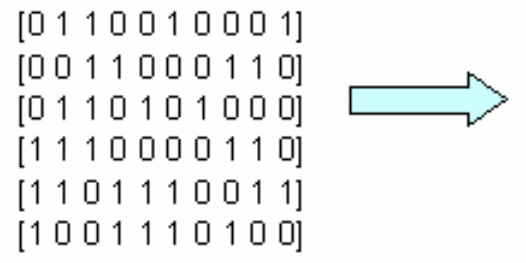

[0110010001]

[0011000110]

[0110101000

[1001110100]

\section{population \\ (real parameters)}

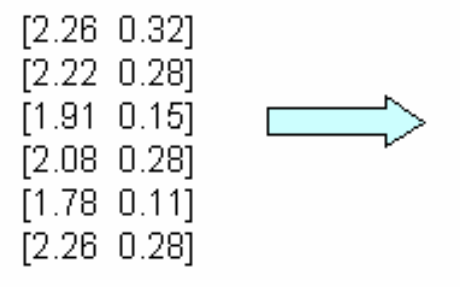

fitness

$F(i)$

170.1553

198.0199

195.9943

187.8672

179.9699

188.6297

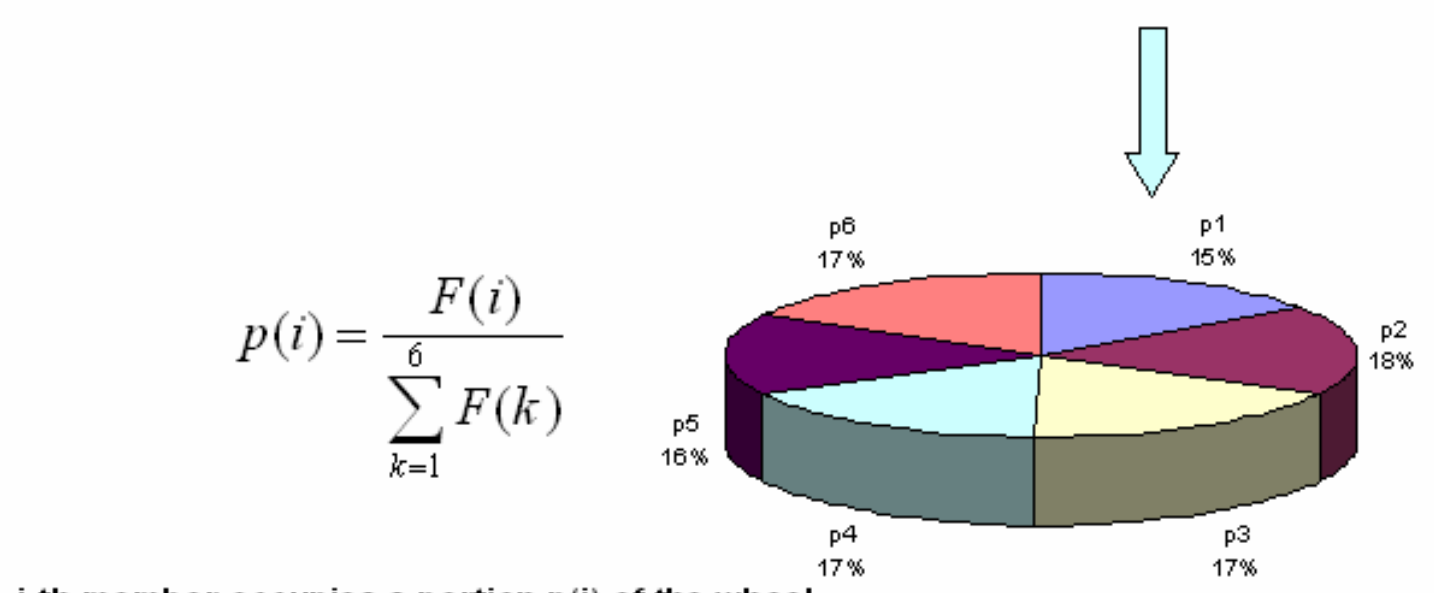

i-th member occupies a portion $p(i)$ of the wheel

\section{Fig. 4.2 Roulette wheel concept of selection}

\subsubsection{Genetic modifications}

The population of a genetic algorithm can be modified in a number of ways. The implementation of GA in this work uses crossover and mutation as the primary methods of genetic evolution, though others exist. These two methods are primarily responsible for the genetic evolution of traits.

\section{Crossover}

Crossover can be viewed as the mating between two parents (two different chromosomes) to produce two new individuals. The two parents are spliced together to create new individuals, as illustrated below.

Parent 1: a b c d e f

Child 1: a b c d E F 


\section{Parent 2: A B C D E F Child 2: A B C D e f}

Two of the parent's genes (e f and E F) were exchanged to create children. The position where crossover occurs is determined randomly and may incorporate any number of genes.

\section{Mutation}

Mutation is an evolutionary instance where a new trait, not inherited from any parent, occurs in the population. Again, a randomly selected bit will change from a zero to a one, or vice versa, to introduce new values of parameters into the population. Mutation is important because a population can become "inbred" and too similar in the wrong direction through pure crossover. Mutations alter only a small fraction of the strings. The probability of mutation can be set as low as roughly one in every 10,000 symbols flips from 0 to 1 , or vice versa. Mutation alone does not generally advance the search for a answer, but it does provide assurance against the development of a uniform population incapable of undergoing further evolution.

The probability of crossover versus mutation is important to finding convergence in the system. However, there is no set answer to how often each should occur. The right balance is particular to the problem and must be selected carefully.

It is also of interest to note that not all mutations and crossovers will be beneficial. Some will not improve the population and will not continue to subsequent generations.

\subsection{Genetic Algorithms in Detection of Embedded Tumors}

The concept of genetic algorithms has been modified for use with the problem of detecting embedded tumors. The potential solution (chromosome) contains the tumor height and its heat generation rate as the two genes. In the current implementation of the code, 5 bits are used to represent each gene. Therefore, each chromosome has 10 bits. This representation is shown graphically in Fig. 4.3. The population size has been chosen to be 20. The code is currently written to perform a set number of iterations. 


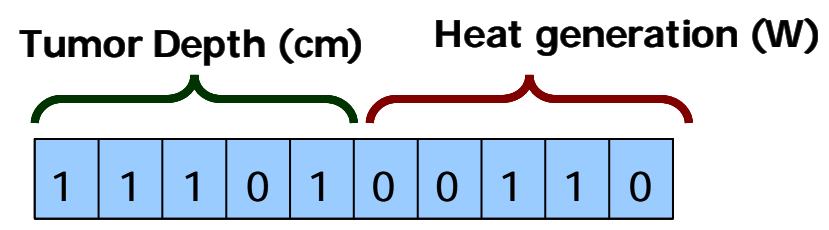

Fig. 4.3 Representation of the solution.

The parameters contained in each chromosome are changed from their binary representation to floating point representation using:

$$
P_{i}=a+\frac{b-a}{2^{n b}-1}(m)
$$

where

$\mathrm{P}_{i}=$ decimal value of $i$-th parameter,

$\mathrm{a}=$ lower limit of the search interval,

$\mathrm{b}=$ upper limit of the search interval,

$\mathrm{nb}=$ number of bits used to represent the parameter, and

$\mathrm{m}=$ the decimal value of the parameter in binary form.

The fitness of each chromosome is evaluated using the sum of the squares objective function given by Equation (3.12). The fitness $f$ of a chromosome is related to the sum of the squares function using:

$$
f=S_{M A X}-S(\boldsymbol{\beta})
$$

where

$\mathrm{S}_{\mathrm{MAX}}=$ maximum value of sum of squares possible, $\mathrm{S}=$ sum of the squares generated using parameter set (chromosome) $\beta$.

The finite difference scheme shown in Fig. 3.1 takes the height and heat generation information from an individual chromosome and determines the subsequent temperature response of the tissue. The closer the finite difference temperature response 
is to the actual temperature response, the lower will be the sum of squares $\mathrm{S}$, and higher would be the fitness of that chromosome. Chromosomes of higher fitness are more likely to be selected to reproduce and contribute their genetic material to the next generation. A probability of mutation of 0.10 and a probability of crossover equal to 0.40 are used to determine how members of the population will reproduce to bring forth the next generation. A flowchart of the solution method is presented in Fig. 4.4.

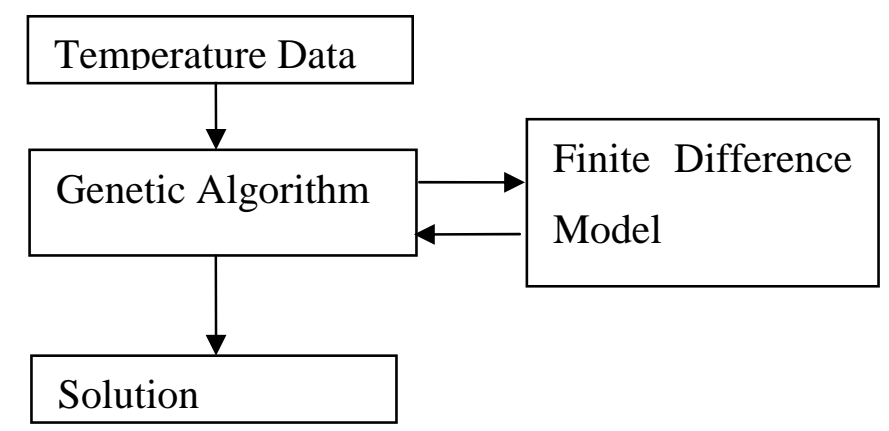

Fig. 4.4 GA based estimation procedure

The computer code for implementing the GA is included in Appendix F. A flowchart showing the estimation methodology in detail is presented in Appendix $\mathrm{H}$. 


\section{Chapter 5 Numerical and Experimental Validation}

\subsection{Numerical Validation}

\subsubsection{Validation of Finite Difference Model}

The numerical finite difference model was described in Section 3.1. In order to establish the accuracy of the model, a commercial Finite Element Method (F.E.M.) package called ANSYS was utilized. A three-dimensional (3D) ANSYS model was generated with the heater embedded $0.03 \mathrm{~m}$ below the surface and having a heat generation of $0.12 \mathrm{~W}$. The finite difference code was run using the same parameters that were used to generate the ANSYS model. The property values used in this evaluation, taken from Werner and Buse(1988), are summarized in Table 5.1.

Table 5.1 Property values used for Evaluation of the Model

\begin{tabular}{|l|l|l|}
\hline Property & Value & Units \\
\hline Thermal conductivity & 0.48 & $\mathrm{~W} / \mathrm{m}-{ }^{\circ} \mathrm{C}$ \\
\hline Heat generation rate & 700 & $\mathrm{~W} / \mathrm{m}^{3}$ \\
\hline Convection coefficient & 13.5 & $\mathrm{~W} / \mathrm{m}^{2}{ }^{\circ} \mathrm{C}$ \\
\hline Ambient temperature & 21.5 & ${ }^{\circ} \mathrm{C}$ \\
\hline
\end{tabular}

\subsubsection{Validation of Parameter Estimation Program}

In order to achieve confidence and check for errors in the parameter estimation program described in Chapter 4, the program was tested with simulated data. The properties used for these simulations are given in Table 5.1. The model was run with specified values for the depth and the power of the heat source. These values were selected so that the effect of varying both the parameters can be observed. Random errors 
of $\pm 0.1{ }^{\circ} \mathrm{C}$ were added to the temperature output from the model in order to create a simulation of experimental data. This was the maximum error expected in the actual data to be obtained using a thermal imaging camera. The simulated data were then used in the estimation program. It was expected that the parameters given by the estimation program should be close to the parameter values that were used to produce the data. Any bias error should be eliminated since the parameter estimation program uses the same numerical model that was used to generate the simulated data. After running the estimation procedure, the percentage errors in each location were calculated by using the total height of the cylinder, $0.064 \mathrm{~m}$, as the base value. The percentage errors in the power were calculated using the maximum expected power, $1.27 \mathrm{~W}$, as the base value. 


\subsection{Experimental Validation}

Several experiments were carried out in order to validate the ability of the estimation procedure to accurately predict the magnitude and depth of an embedded heat source. For each parameter set, two runs of the experiment were conducted in order to gain confidence in the recorded data. Data from both runs were then used with the estimation procedure in order to predict the desired parameters. A parameter set in this study is defined as a particular combination of the depth of heat source and its heat dissipation. Experiments were conducted for a total of six different parameter sets in order to explore the effect of both the depth of the heater and its heat dissipation. The experiments were designed to meet the following criteria:

1. The geometry of the medium needed to be cylindrical to match the finite difference solver developed in the cylindrical coordinate system. This was done in order to simplify the problem. Reducing the problem from three to two dimensions greatly reduces the amount of temperature data to be considered and the computational resources involved in running the parameter estimation program. The model and the estimation procedures can also be used with multidimensional complex geometries.

2. The heat source needed to be circular in shape, have a uniform heat dissipation over its surface area, and placed at the center of the cylinder. This was to ensure that conditions of symmetry in the angular direction can be used to justify the two-dimensional treatment of the problem.

3. Since the surface temperatures in thermal imaging are sensitive to the temperature of the environment, the experiment needed to be performed in a room maintained at a constant temperature. In a clinical setting, the room is maintained between 18-22 ${ }^{\circ} \mathrm{C}$ and held to within $1{ }^{\circ} \mathrm{C}$ of change (Amalu, 2003). The illumination, sunlight, and humidity of the room are carefully controlled. 
4. The temperature was to be measured using both infrared camera and the thermocouples. Since the temperature data were obtained using an infrared camera, thermocouples were also needed to calibrate the camera. In a clinical setting, cameras are usually calibrated by recording the temperature of a black body that is maintained at a known temperature value.

The experimental apparatus and setup are presented in Section 5.1. The procedures and test conditions of this experiment are described in Section 5.2.

\section{Experimental Setup}

Figures 5.1 and 5.2 show the configuration used to perform the experiment.

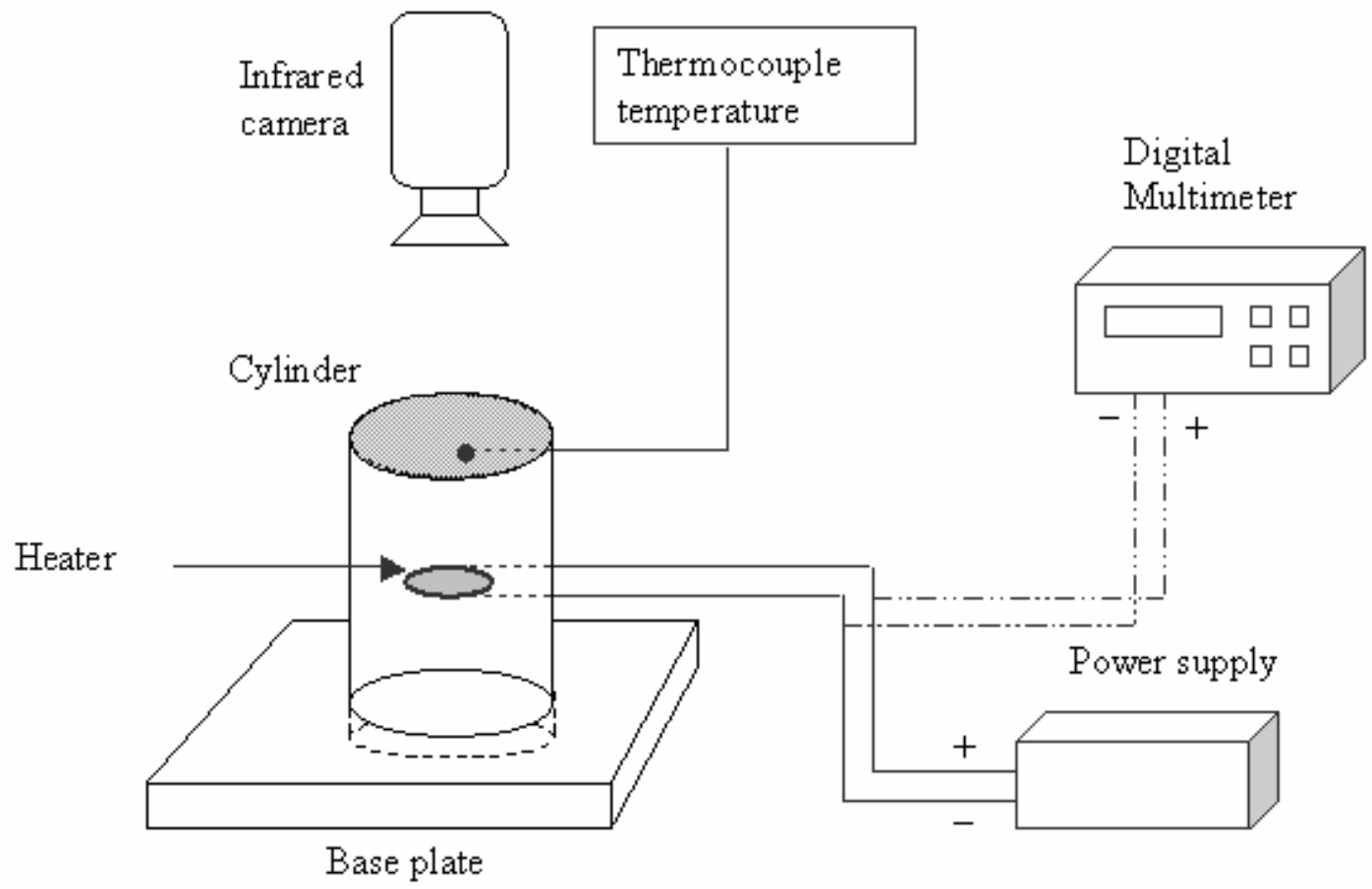

Fig. 5.1 Schematic of the experimental setup 


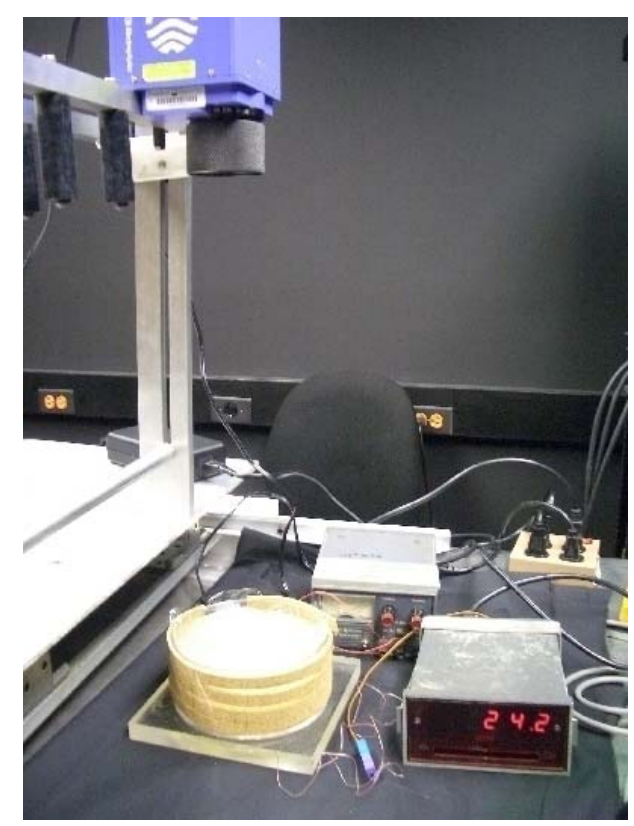

Fig. 5.2 Photograph of the experimental setup

The setup consisted of a lexan circular cylinder filled with agar phantom and mounted on top of a square base plate.The cylinder and base plate assembly, along with its dimensions, is shown in Fig. 5.3.

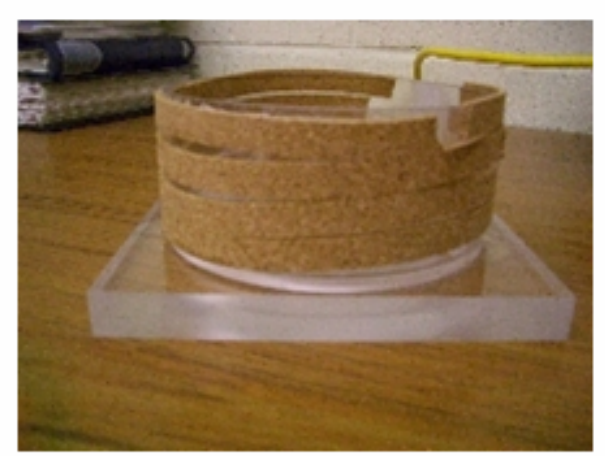

(a)

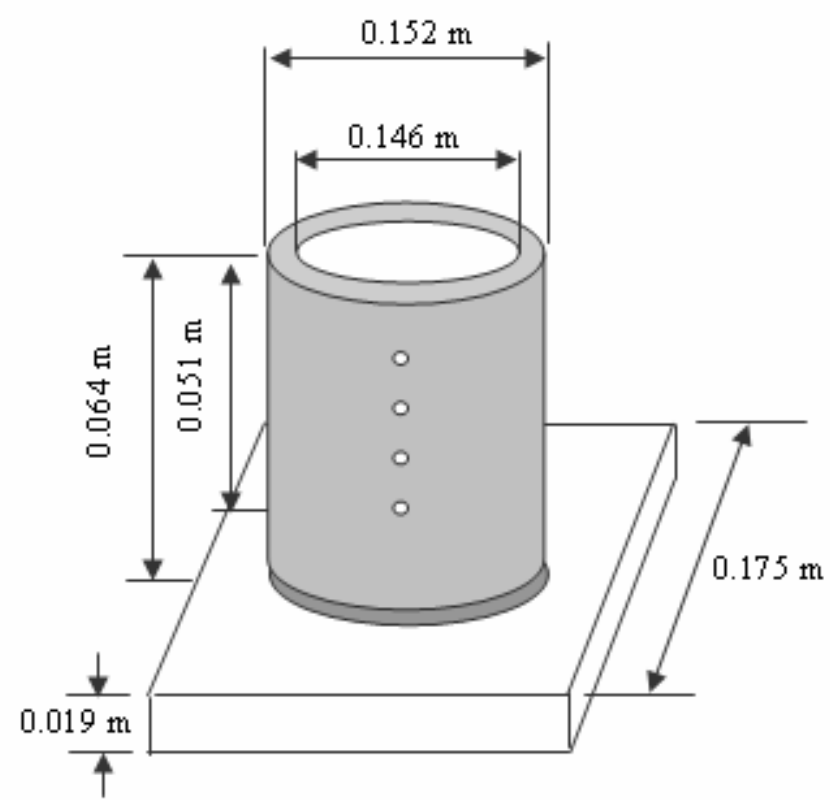

(b)

Fig. 5.3 Figure showing the (a) photograph and (b) dimensions of the cylinder and base plate assembly 
Four equally spaced holes were drilled into the cylinder at different depths for inserting the heater wire. The cylinder was covered with $0.02 \mathrm{~m}$ thick natural cork strip insulation around its circumference. The cylinder was insulated on all sides except the top surface that was exposed to convection.

A resistance heater was embedded in agar and connected to a 0-60 V DC power supply. The resistance heater is shown in Fig. 5.4. The heater has a radius of $0.023 \mathrm{~m}$ and a resistance of $32 \mathrm{Ohms}$. A digital multimeter was set up to probe the voltage across the heater.

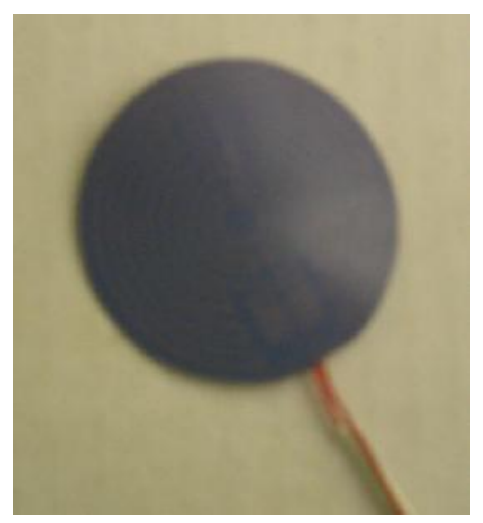

Fig. 5.4 The resistance heater used as the heat source in the experiment

The PV-320T model of the infrared camera from Electrophysics was used for thermal imaging. This camera is shown in Fig. 5.5. Specifications for the camera can be found in Appendix I1.

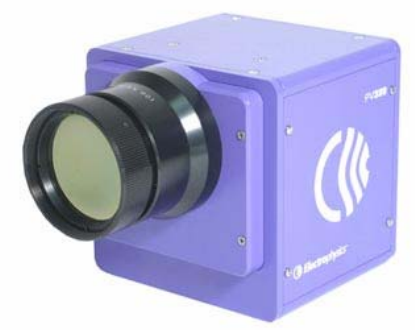

Fig. 5.5 The Infrared camera used in the experiment 


\section{Experimental Procedures}

A 1.0 percent solution of Difco Agar, Technical $(C)$ was prepared by dissolving agar in boiling water. The solution was then allowed to cool for a few hours until it acquired semi-solid jelly-like consistency. The agar was poured into the insulated cylinder and the resistance heater was embedded in the agar at the required depth. Care was taken to ensure that the heater was placed exactly at the center of the cylinder and it is not disturbed when agar is poured on it. The heater wire protruding through the hole in the cylinder was connected to the power supply. The power supply was used to adjust the voltage applied across the heater. By changing the voltage across the heater, the power dissipated by the heater could be adjusted to desired value. A total of six experiments were carried out, grouped into experimental sets A and B. The combinations of the depth of the heater and its power dissipation for each of the 6 experiments are given in Table 5.2 .

Table 5.2 Parameters used for different experimental runs

\begin{tabular}{|l|l|l|}
\hline Exp. & Heater Depth (m) & Power (W) \\
\hline A1 & 0.013 & 0.12 \\
\hline A2 & 0.013 & 0.78 \\
\hline A3 & 0.025 & 0.12 \\
\hline A4 & 0.025 & 0.78 \\
\hline B1 & 0.038 & 1.12 \\
\hline B2 & 0.051 & 1.12 \\
\hline
\end{tabular}

The parameters for each of these six experiments are shown graphically in Fig. 5.6. The curve in Fig. 5.6 was prodcued using the numerical model described in Section 3.1. For a given depth of the heater, the curve shows the minimum power level that is necessary to produce a $0.5{ }^{\circ} \mathrm{C}$ temperature differential on the surface of the cylinder. 
Therefore, for any combinations of the parameters that lie below the curve, the heater would not detectable based on the infrared image.

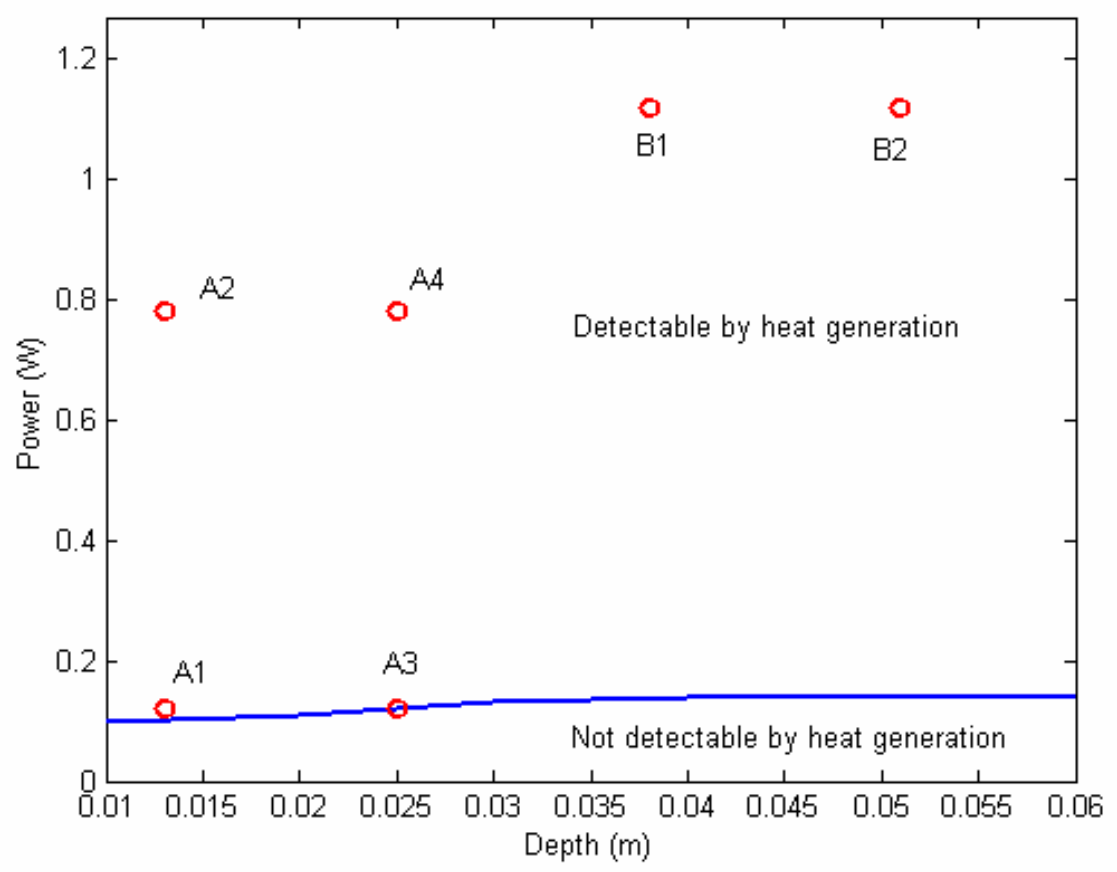

Fig. 5.6 The parameters used for different experimental runs and the limits of steady-state detection of the heater assuming a mimimum temperature rise of 0.5 degrees on the surface

The cylinder was aligned with the infrared camera such that its top surface was visible in the infrared images acquired using the Velocity software. For each experiment, the setup was left undisturbed for about 4 hours after turning on the power to ensure a steady-state temperature field in the specimen.

The infrared camera was calibrated with the help of thermocouple temperature data. The thermocouple was placed on the surface of the cylinder and used for measuring several steady-state temperature values. These steady-state temperatures were also captured by the infrared camera. The average pixel gray scale values at the location of the thermocouple were plotted against the thermocouple temperatures in degree Celsius. The resulting interpolation curve is shown in Fig. 5.7. This method eliminated the need to know the emissivity of the agar specimen. In a clinical setting, it is important to 
accurately know the emissivity of the human skin. Steketee (1973) has described a method in which skin radiation is compared with blackbody radiation. Fujimasa et al. (1973) have pointed out emissivity differences in skin covered with tinctures and paints.

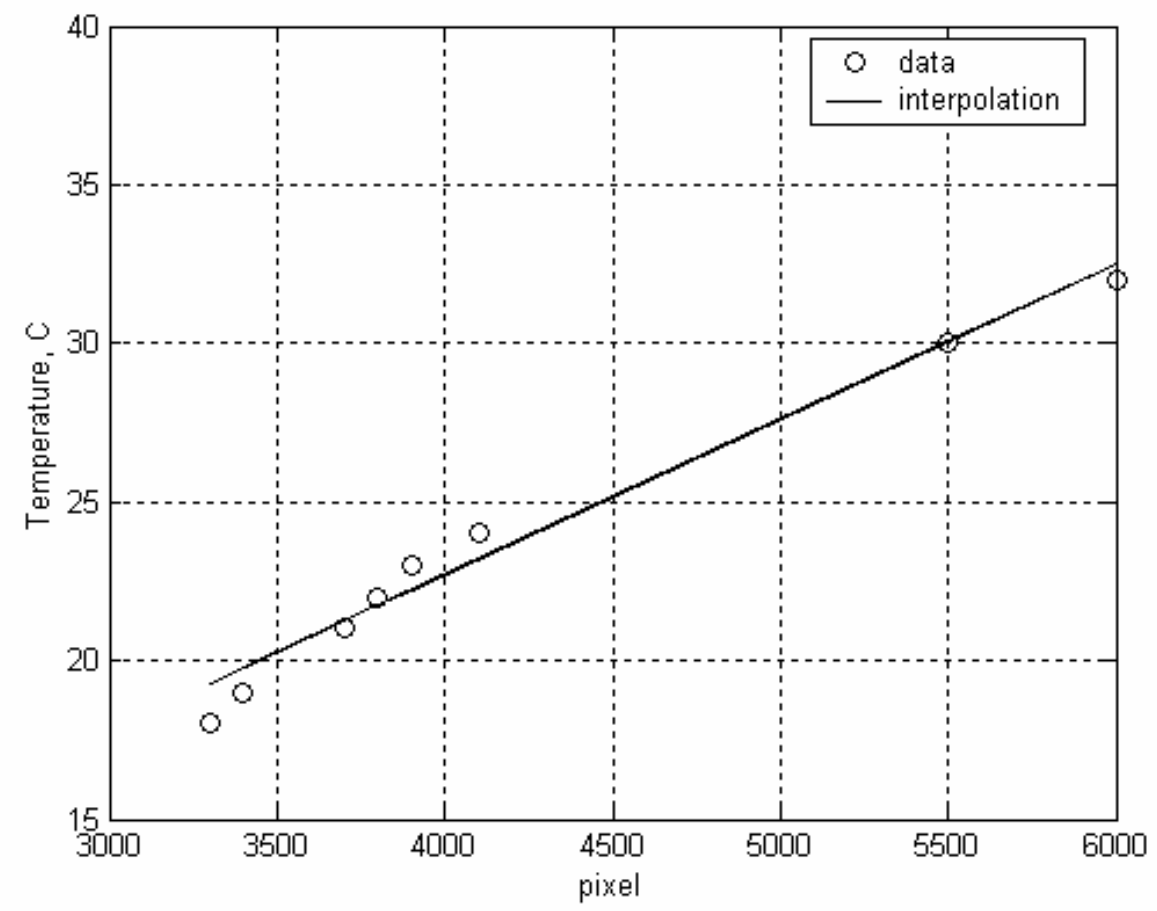

Fig. 5.7 Infrared camera calibration using thermocouple

The data from the infrared camera were reduced using the process shown in Fig. 5.8. Each infrared image was stored in a digital ".fts" file format. This image file was used to obtain a matrix of gray scale values that was stored in a ASCII text file. A MATLAB program was written for locating the boundaries and the center of the cylindrical surface, and generating coordinates of points of interest in this $(r, \theta)$ coordinate system. The program read in the gray scale values at the points of interest, and converted them to temperature in degree Celsius using the calibration data. The computer code for this program can be found in Appendix G. 


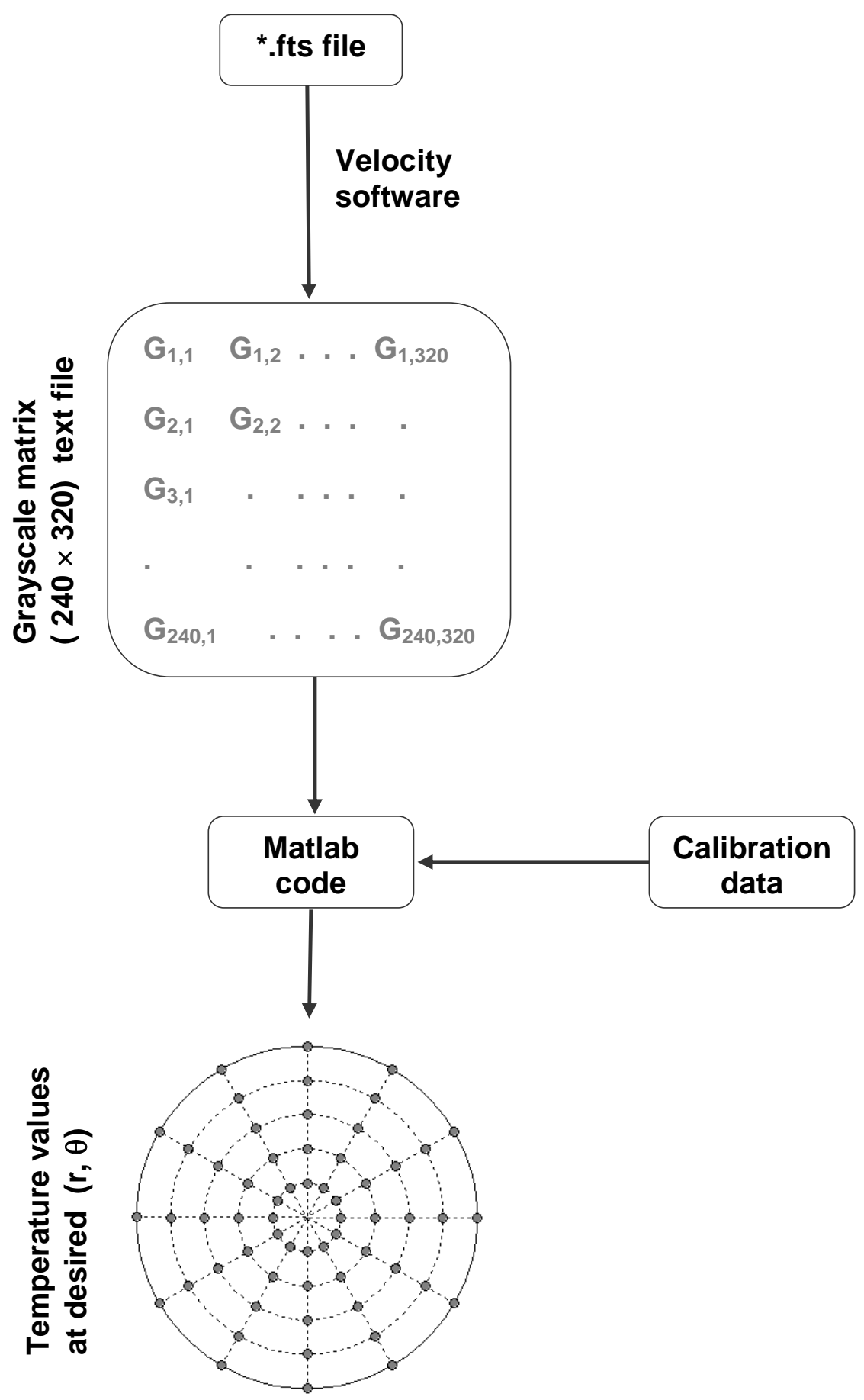

Fig. 5.8 Infrared data reduction

Since the temperature at any point on the surface of the cylinder should depend only on the radial location of that point, and not on the angular location, data were taken 
along several radial lines placed at equal angles from each other. Radial lines run from the center of the cylinder to its circumference, as shown in Fig. 5.9.

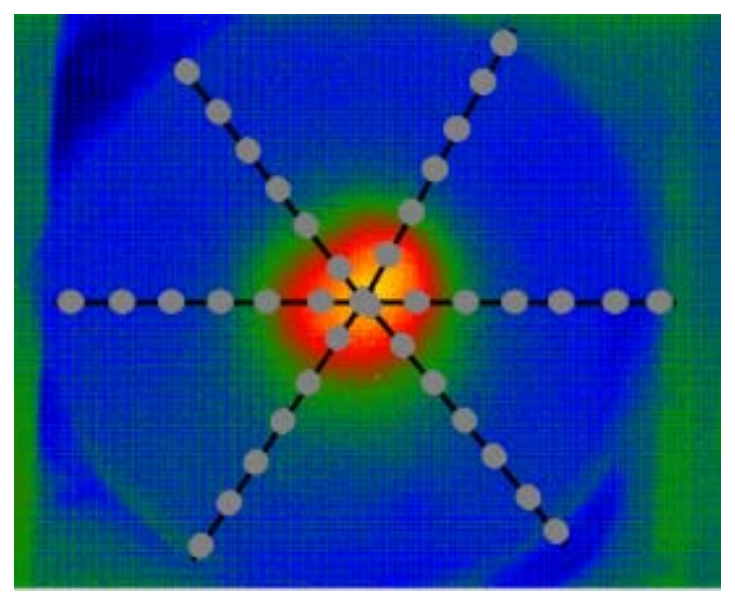

Fig. 5.9 A thermal image showing the surface of the cylinder and some radial lines along which the temperature data are taken.

To obtain an accurate estimate of the temperature at a given distance from the center of the cylinder surface, the temperatures at several points around the circumference were averaged to get a mean temperature. The confidence intervals were calculated using Equation (3.21). The data from the experiments were used to determine the average convection coefficient and the thermal conductivity of agar by using the BoxKanemasu minimization procedure. A flowchart showing the estimation of these thermal properties is shown in Fig. 5.10. The computer code that implements this procedure can be found in Appendix E2.

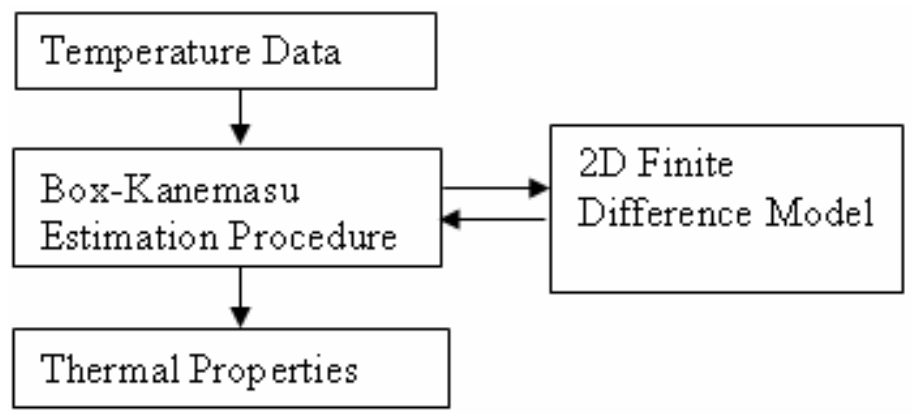

Fig. 5.10 Estimation of thermal properties 


\section{Chapter 6 Numerical and Experimental Results}

This chapter presents the results of both the numerical simulations and the experiments. First, the results of numerical model validation are presented in order to establish the validity of the model. Presented next are the results of the parameter estimation program validation using simulated data with random errors. Finally, the results from the experiments are presented. The results obtained from several different experimental runs are graphed, showing both the experimental curves and the corresponding theoretical curves obtained using computer program.

\subsection{Numerical Model Validation Results}

The three-dimensional (3D) ANSYS model is shown in Fig. 6.1 below. The surface temperature profiles obtained using ANSYS were compared with the corresponding temperature profiles from the finite difference model by superimposing the two curves on the same plot. This plot is shown in Fig. 6.2. It can be seen from Fig. 6.2 that the two temperature profiles closely match although they were produced using two independent computer programs. The maximum error in the two temperature profiles is approximately $0.1{ }^{\circ} \mathrm{C}$. It can therefore be concluded that the two-dimensional finite difference model accurately represents heat flow in the 3D geometry of a cylinder. 


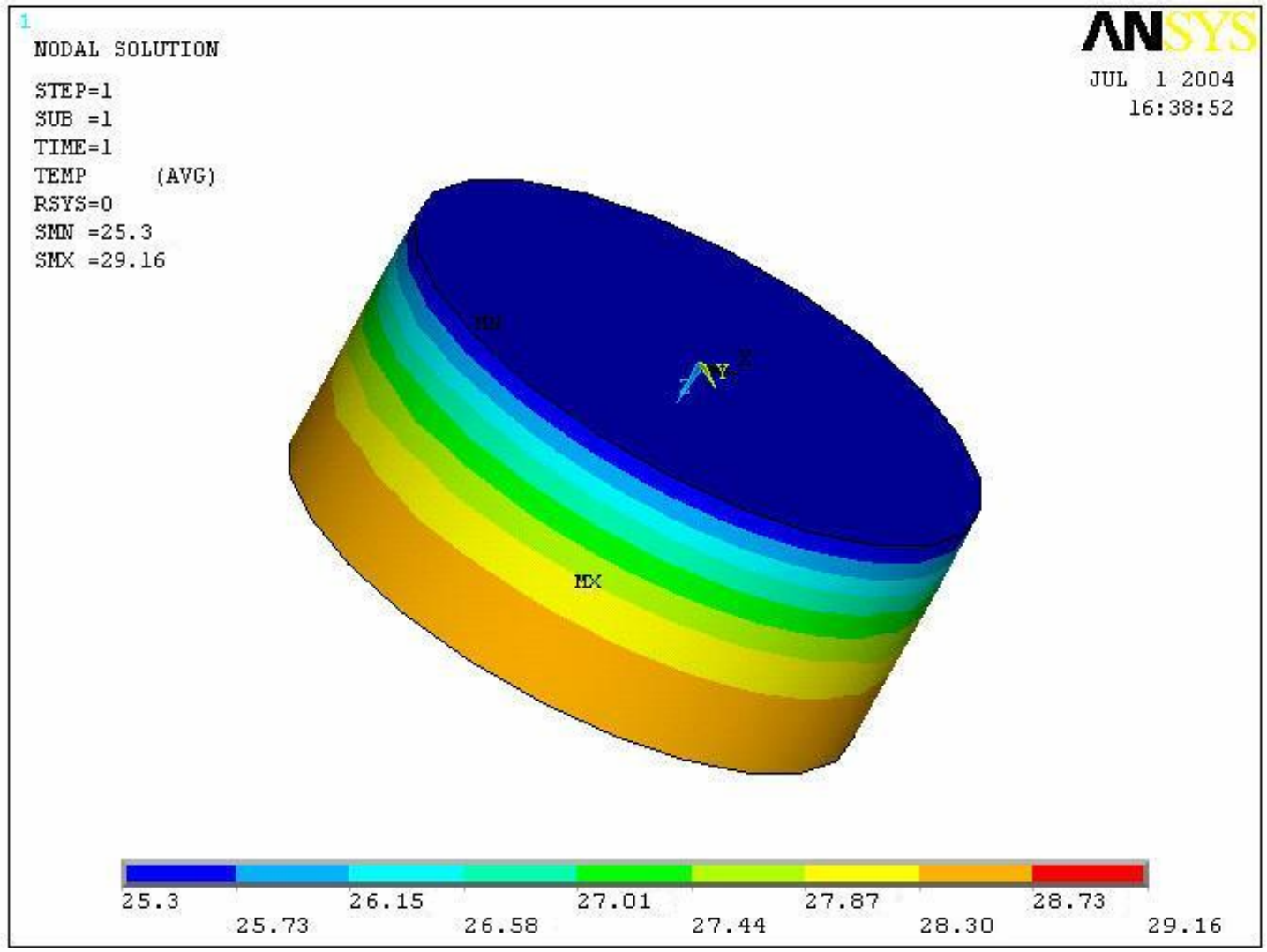

Fig. 6.1 3D ANSYS model showing the temperature distributions in a cylinder with an embedded heat source

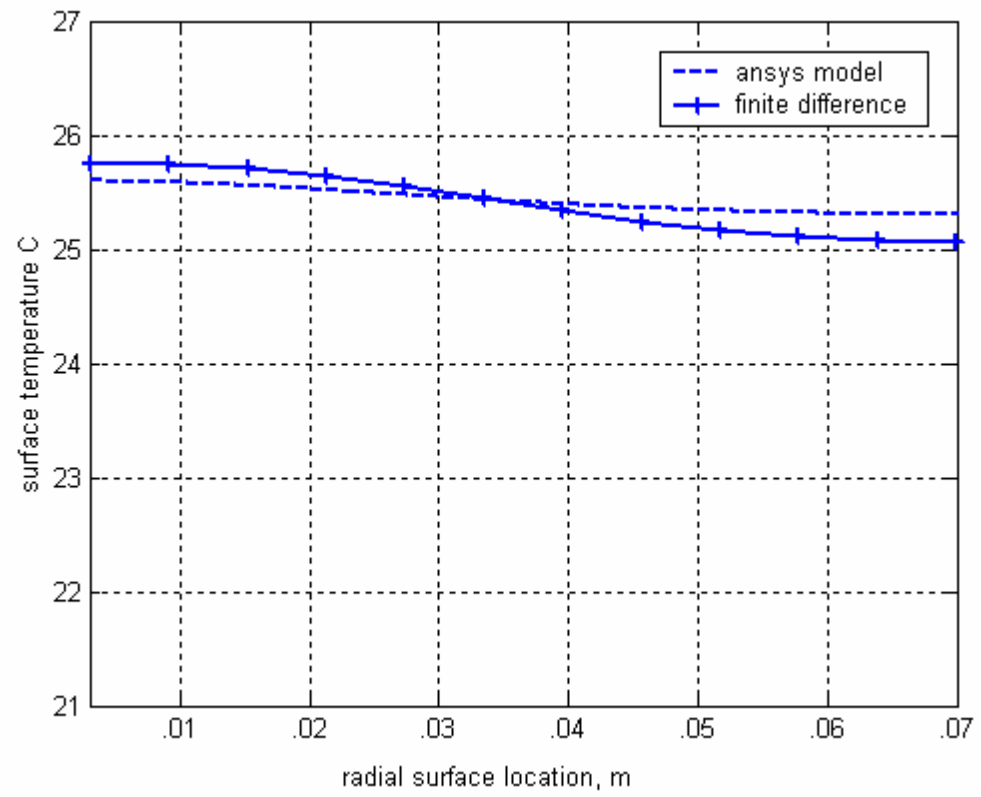

Fig. 6.2 Temperature profiles obtained using finite difference model and ANSYS software 


\subsection{Parameter Estimation Program Validation Results}

The results of the test runs are shown in Fig. 6.3. Each of these figures show both the simulated experimental data and the corresponding temperature data obtained using predicted parameters. The results of these test cases are summarized in Table 6.1, along with the absolute and the percentage errors in the parameters. It can be seen from these cases that the parameter estimation program performs well considering the random errors in the simulated data. The maximum error in the depth is within 5 percent, while the maximum error in heat generation is within 3 percent.

Table 6.1 Results of Simulations

\begin{tabular}{|c|c|c|c|c|c|c|c|c|}
\hline \multirow{2}{*}{ Case } & \multicolumn{2}{|c|}{ Actual } & \multicolumn{2}{c|}{ Estimated } & \multicolumn{2}{c|}{ Absolute Error } & \multicolumn{2}{c|}{ Percent Error } \\
\cline { 2 - 9 } & $\begin{array}{c}\text { Depth } \\
(\mathrm{m})\end{array}$ & $\begin{array}{c}\text { Power } \\
(\mathrm{W})\end{array}$ & $\begin{array}{c}\text { Depth } \\
(\mathrm{m})\end{array}$ & $\begin{array}{c}\text { Power } \\
(\mathrm{W})\end{array}$ & $\begin{array}{c}\text { Depth } \\
(\mathrm{m})\end{array}$ & $\begin{array}{c}\text { Power } \\
(\mathrm{W})\end{array}$ & Depth & Power \\
\hline a & 0.025 & 0.50 & 0.027 & 0.53 & 0.002 & 0.03 & 3.1 & 2.4 \\
\hline b & 0.035 & 0.50 & 0.036 & 0.51 & 0.001 & 0.01 & 1.6 & 0.8 \\
\hline c & 0.045 & 1.00 & 0.048 & 1.02 & 0.003 & 0.02 & 4.7 & 1.6 \\
\hline
\end{tabular}




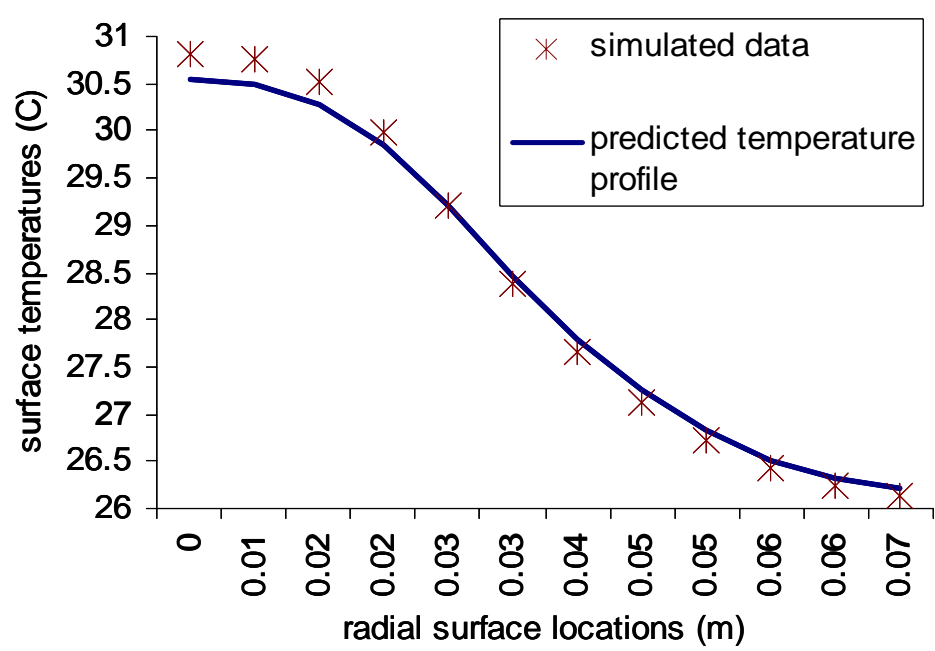

(a)

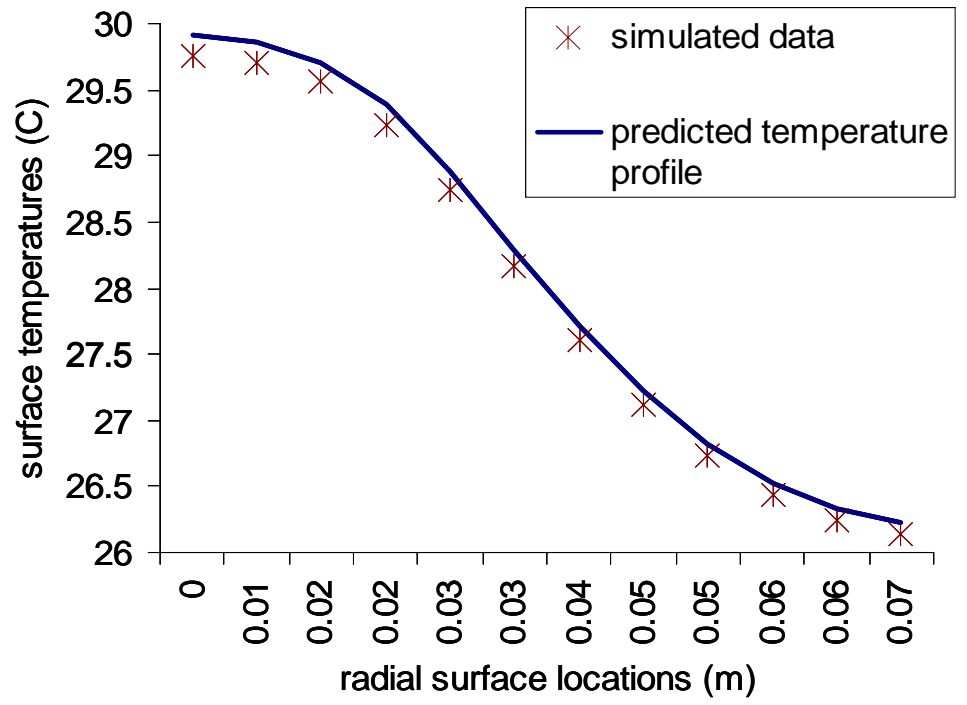

(b)

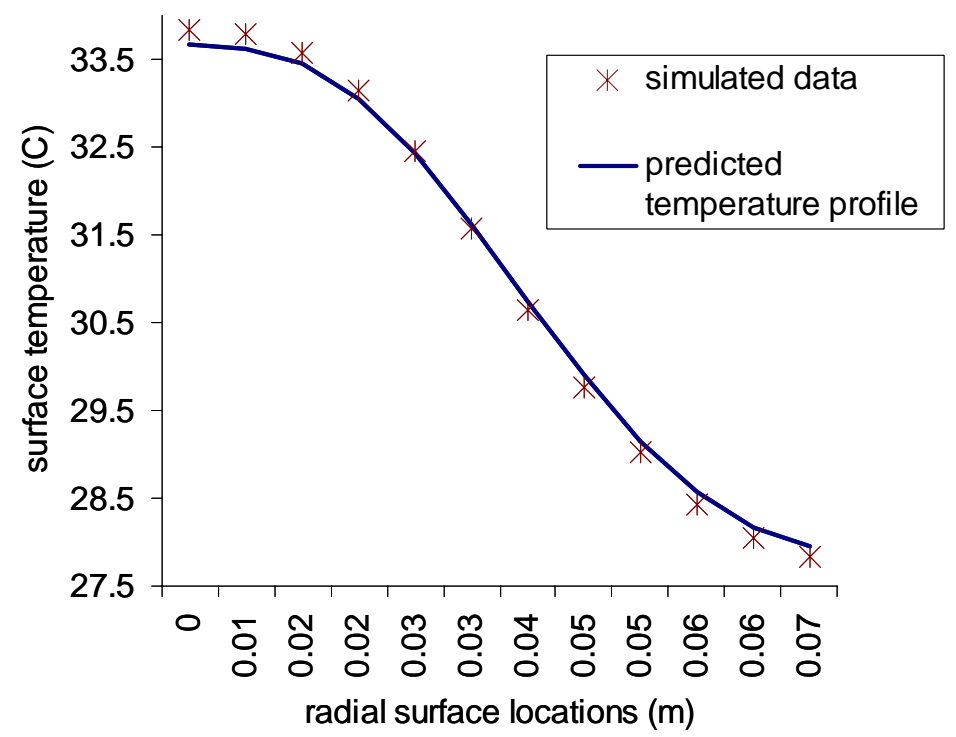

(c)

Fig. 6.3 Plot showing simulated data and the resulting temperature distribution predicted using estimation program for cases $a, b$ and $c$. 


\subsection{Results of Experiments}

This section presents the results from the six experiments. Since the last two experiments were conducted at a different time than the first four experiments, they share different convection coefficient and thermal conductivity values. The difference in thermal conductivity arises primarily from the difference in quality of the agar in use. Therefore, these are reported separately under Experiment Set B. The uncertainty in convection coefficient and thermal conductivity parameters in the direct numerical model clearly effects the solution, yet they are not the parameters that we are seeking. In order to study the effect of these parameters on the model, results from a sensitivty analysis are presented in Section 6.4.

\section{Experiment Set A}

This section presents the results from the first set of experiments. The average convection coefficient was found to be $29.0 \pm 4.0 \mathrm{~W} / \mathrm{m}^{2}{ }^{\circ} \mathrm{C}$. The ambient air temperature was $21.5^{\circ} \mathrm{C}$. Since the agar solution used for this set of experiments had appearance of jelly and made of 99 percent water, the value of its thermal conductivity was taken to be $0.61 \mathrm{~W} / \mathrm{m}-{ }^{\circ} \mathrm{C}$, the same as the thermal conductivity of water. It was found that this value matches the observed temperature profiles well.

The surface temperature data obtained from the experiments were used to predict the depth and the power of the heater using the GA. The results are summarized in Table 6.2. These results show excellent agreement between actual and predicted parameters, with percentage errors in depth within 6 percent, and percentage errors in power within 3 percent. Fig. 6.4 through Fig. 6.7 show the surface temperature data from the experiments, and the temperature profile using the parameters predicted by the GA. 
Table 6.2 Results of Experimental Set A

\begin{tabular}{|c|c|c|c|c|c|c|c|c|c|}
\hline \multirow[b]{2}{*}{ Exp. } & \multirow[b]{2}{*}{ Run } & \multicolumn{2}{|c|}{ Actual } & \multicolumn{2}{|c|}{ Estimated } & \multicolumn{2}{|c|}{ Absolute Error } & \multicolumn{2}{|c|}{ Percent Error } \\
\hline & & $\begin{array}{c}\text { Depth } \\
\text { (m) }\end{array}$ & $\begin{array}{c}\text { Power } \\
\text { (W) }\end{array}$ & $\begin{array}{c}\text { Depth } \\
\text { (m) }\end{array}$ & $\begin{array}{c}\text { Power } \\
\text { (W) }\end{array}$ & $\begin{array}{c}\text { Depth } \\
\text { (m) }\end{array}$ & $\begin{array}{c}\text { Power } \\
\text { (W) }\end{array}$ & Depth & Power \\
\hline \multirow{2}{*}{ A1 } & 1 & \multirow{2}{*}{0.013} & \multirow{2}{*}{0.12} & 0.016 & 0.14 & 0.003 & 0.02 & 4.7 & 1.6 \\
\hline & 2 & & & 0.017 & 0.14 & 0.004 & 0.02 & 6.3 & 1.6 \\
\hline \multirow{2}{*}{$\mathrm{A} 2$} & 1 & \multirow{2}{*}{0.013} & \multirow{2}{*}{0.78} & 0.011 & 0.76 & 0.002 & 0.02 & 3.1 & 1.6 \\
\hline & 2 & & & 0.012 & 0.79 & 0.001 & 0.01 & 1.6 & 0.8 \\
\hline \multirow{2}{*}{ A3 } & 1 & \multirow{2}{*}{0.025} & \multirow{2}{*}{0.12} & 0.021 & 0.14 & 0.004 & 0.02 & 6.3 & 1.6 \\
\hline & 2 & & & 0.022 & 0.15 & 0.003 & 0.03 & 4.7 & 2.4 \\
\hline \multirow{2}{*}{ A4 } & 1 & \multirow{2}{*}{0.025} & \multirow{2}{*}{0.78} & 0.028 & 0.72 & 0.003 & 0.06 & 4.7 & 4.7 \\
\hline & 2 & & & 0.028 & 0.75 & 0.003 & 0.03 & 4.7 & 2.4 \\
\hline
\end{tabular}




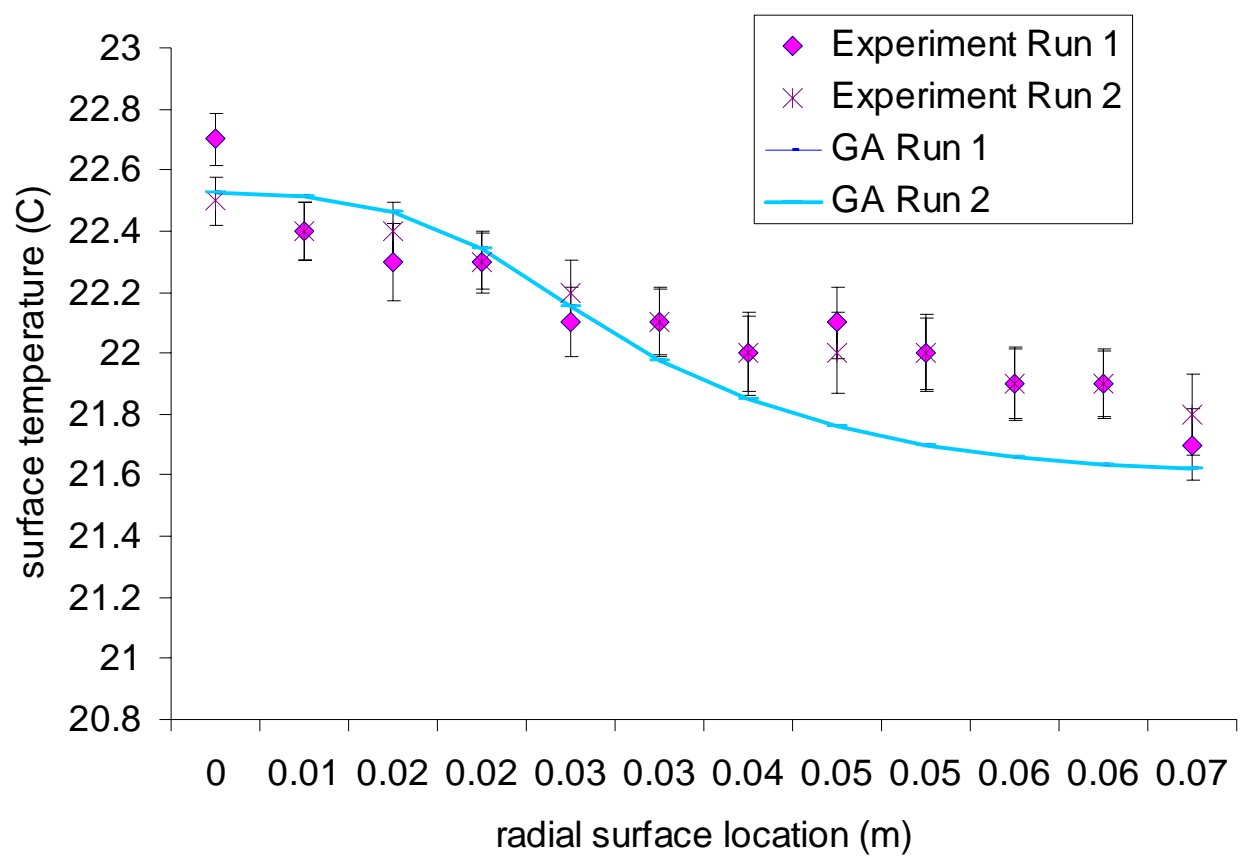

Fig. 6.4 Result of Experiment A1 showing experimental data and predicted temperature profiles

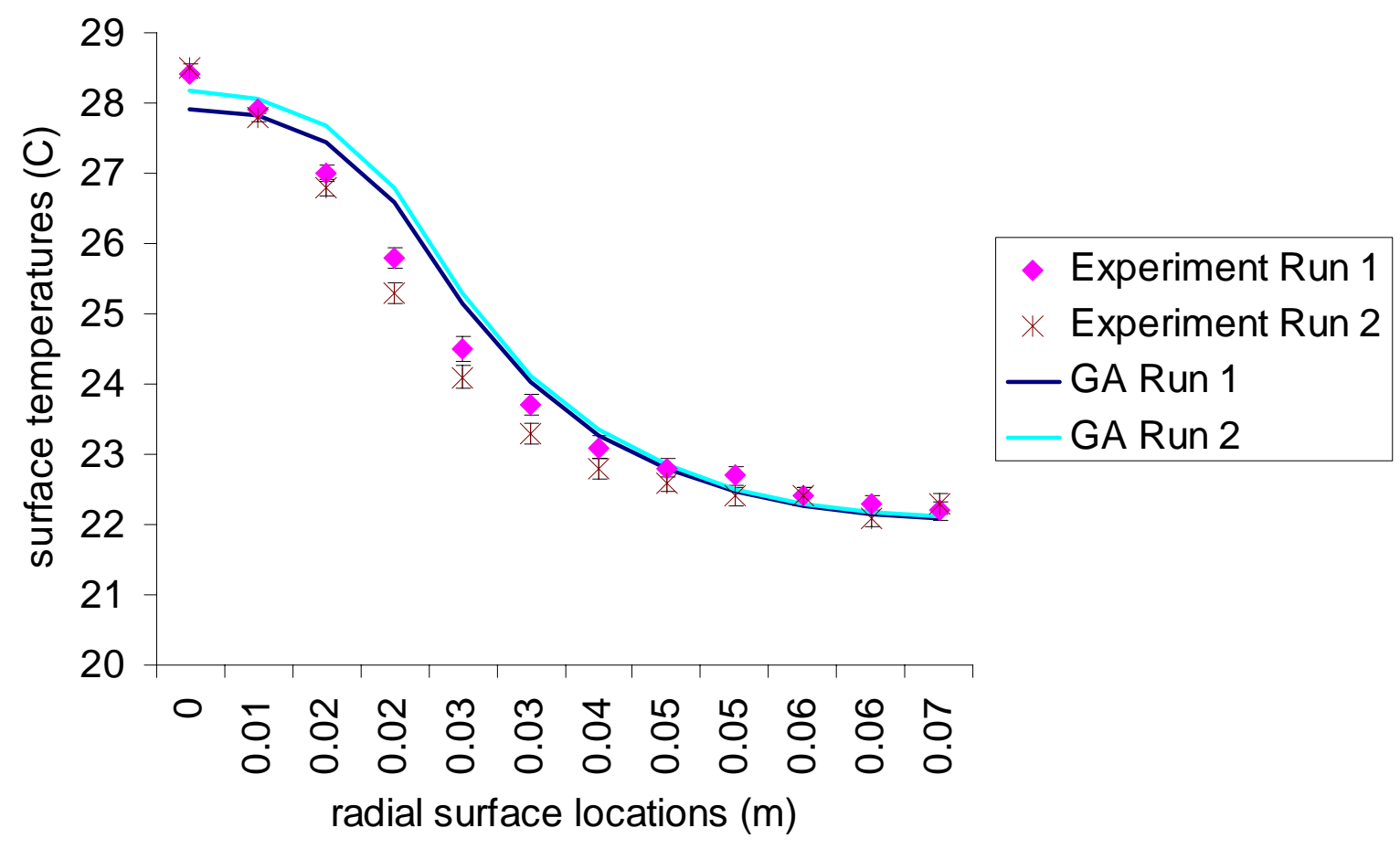

Fig. 6.5 Result of Experiment A2 showing experimental data and predicted temperature profiles 


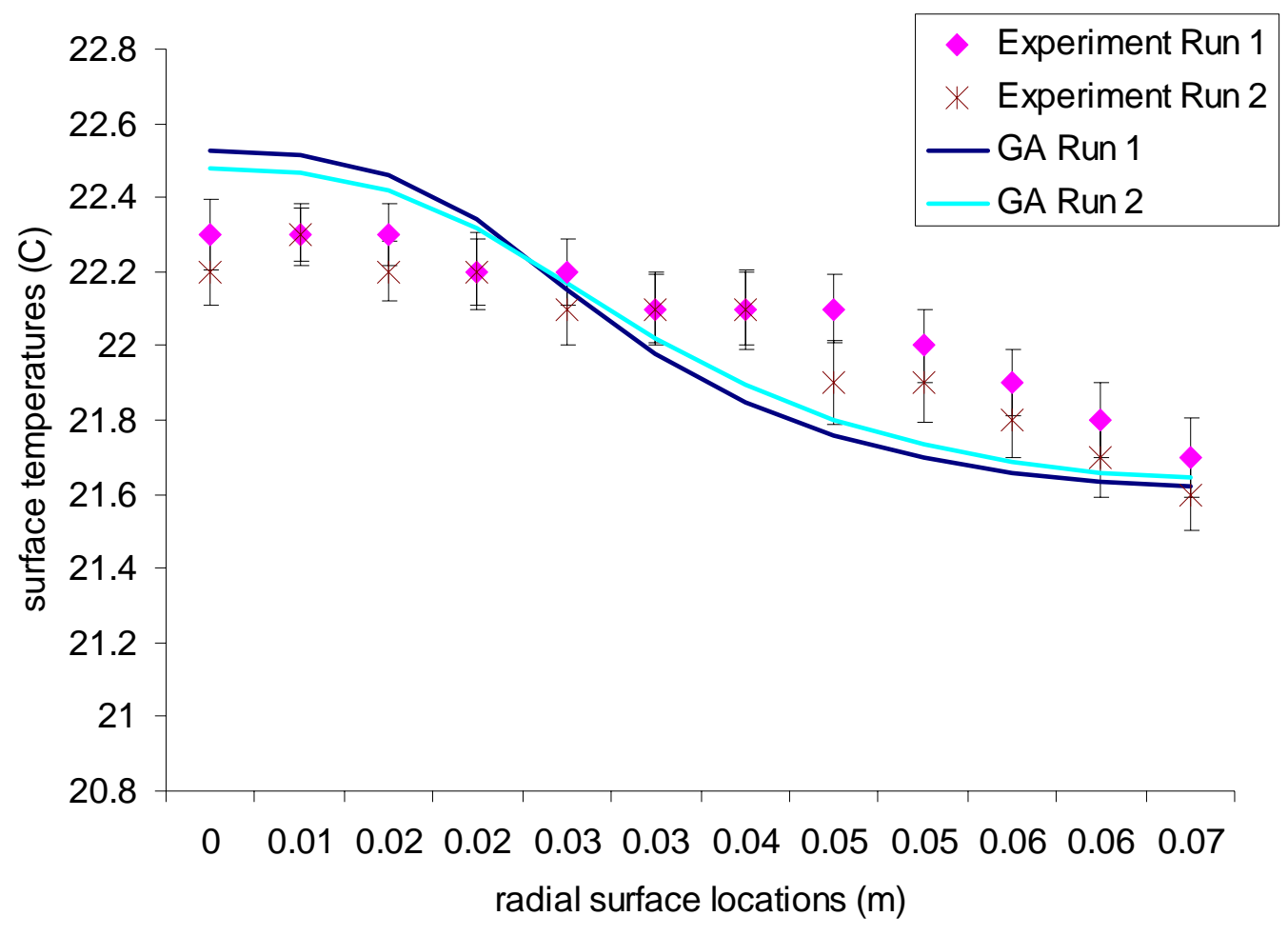

Fig. 6.6 Result of Experiment A3 showing experimental data and predicted temperature profiles

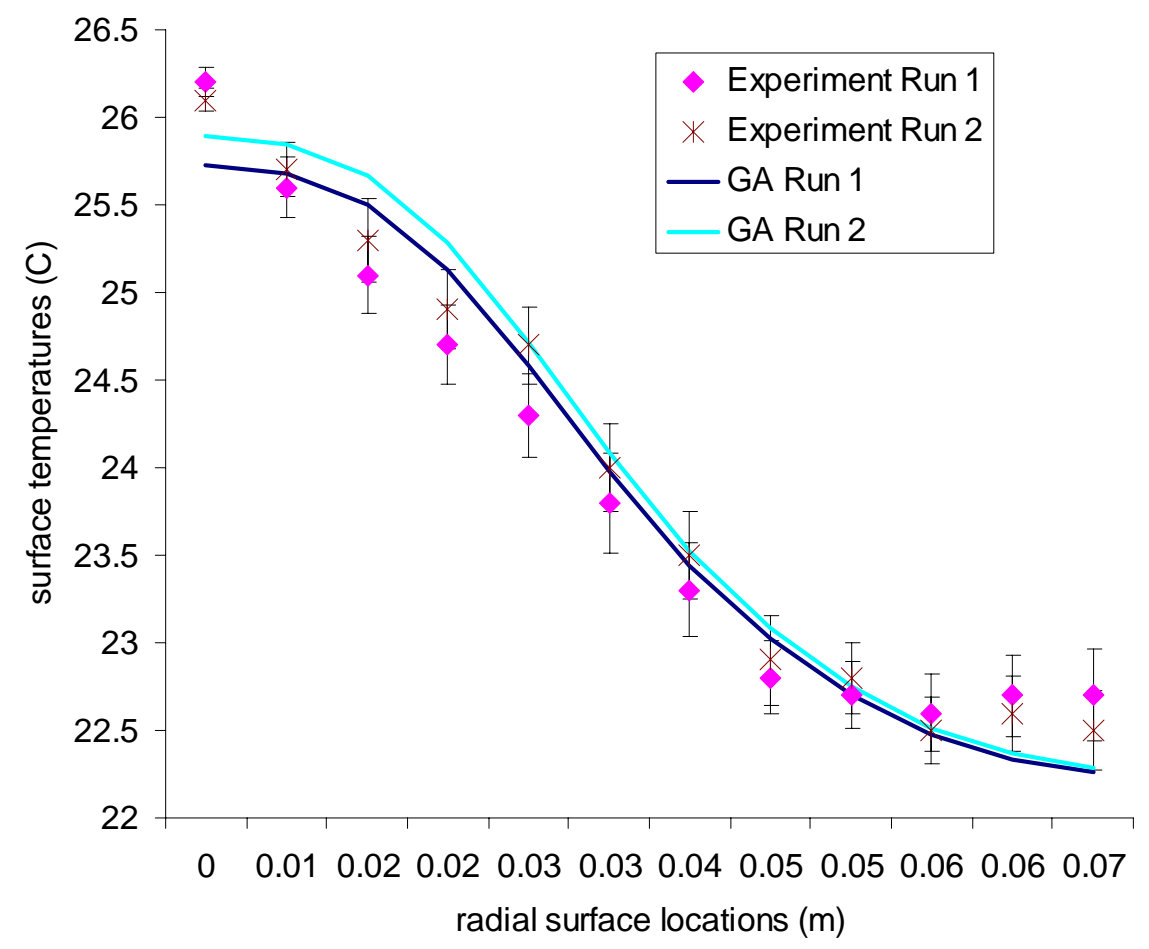

Fig. 6.7 Result of Experiment A4 showing experimental data and predicted temperature profiles 


\section{Experiment Set B}

For the second set of experiments, the experimental data were used to estimate both the average convection coefficient and the average thermal conductivity. The average convection coefficient for this set of experiments was found to be $24.0 \pm 3.0$ $\mathrm{W} / \mathrm{m}^{2}{ }^{\circ} \mathrm{C}$, which is close to the convection coefficient found for the first set of experiments. The ambient air temperature was $21.5^{\circ} \mathrm{C}$. The average thermal conductivity was found to be $3.4 \pm 1.0 \mathrm{~W} / \mathrm{m}-{ }^{\circ} \mathrm{C}$. The most likely cause of this large uncertainty associated with the thermal conductivity is due to the quality of the agar. Solidification of agar can cause large changes in its thermal conductivity. Therefore, it was noted that the quality of agar must be very precisely controlled during any future experiments, and a quantitative method must be devised to assess its quality than a mere visual inspection. The results are summarized in Table 6.3. Overall, these results show a higher percentage error than the parameter estimation results in Experiment Set A. Fig. 6.8 and Fig. 6.9 show the surface temperature data from the experiments, and the temperature profile using the parameters predicted by the GA.

Table 6.3 Results of Experimental Set B

\begin{tabular}{|c|c|c|c|c|c|c|c|c|c|}
\hline \multirow{2}{*}{ Exp. } & \multirow{2}{*}{ Run } & \multicolumn{2}{|c|}{ Actual } & \multicolumn{2}{|c|}{ Estimated } & \multicolumn{2}{|c|}{$\begin{array}{c}\text { Absolute } \\
\text { Error }\end{array}$} & \multicolumn{2}{|c|}{ Percent Error } \\
\hline & & $\begin{array}{l}\text { Depth } \\
\text { (m) }\end{array}$ & $\begin{array}{c}\text { Power } \\
\text { (W) }\end{array}$ & $\begin{array}{l}\text { Depth } \\
\text { (m) }\end{array}$ & $\begin{array}{l}\text { Power } \\
\text { (W) }\end{array}$ & $\begin{array}{c}\text { Depth } \\
\text { (m) }\end{array}$ & $\begin{array}{l}\text { Power } \\
\text { (W) }\end{array}$ & Depth & Power \\
\hline \multirow{2}{*}{ B1 } & 1 & \multirow{2}{*}{0.038} & \multirow{2}{*}{1.12} & 0.038 & 0.98 & - & 0.14 & - & 11.0 \\
\hline & 2 & & & 0.039 & 1.07 & 0.001 & 0.05 & 1.6 & 4.0 \\
\hline \multirow{2}{*}{ B2 } & 1 & \multirow{2}{*}{0.051} & \multirow{2}{*}{1.12} & 0.053 & 1.17 & 0.002 & 0.05 & 3.2 & 4.0 \\
\hline & 2 & & & 0.050 & 1.10 & 0.001 & 0.02 & 1.6 & 1.6 \\
\hline
\end{tabular}




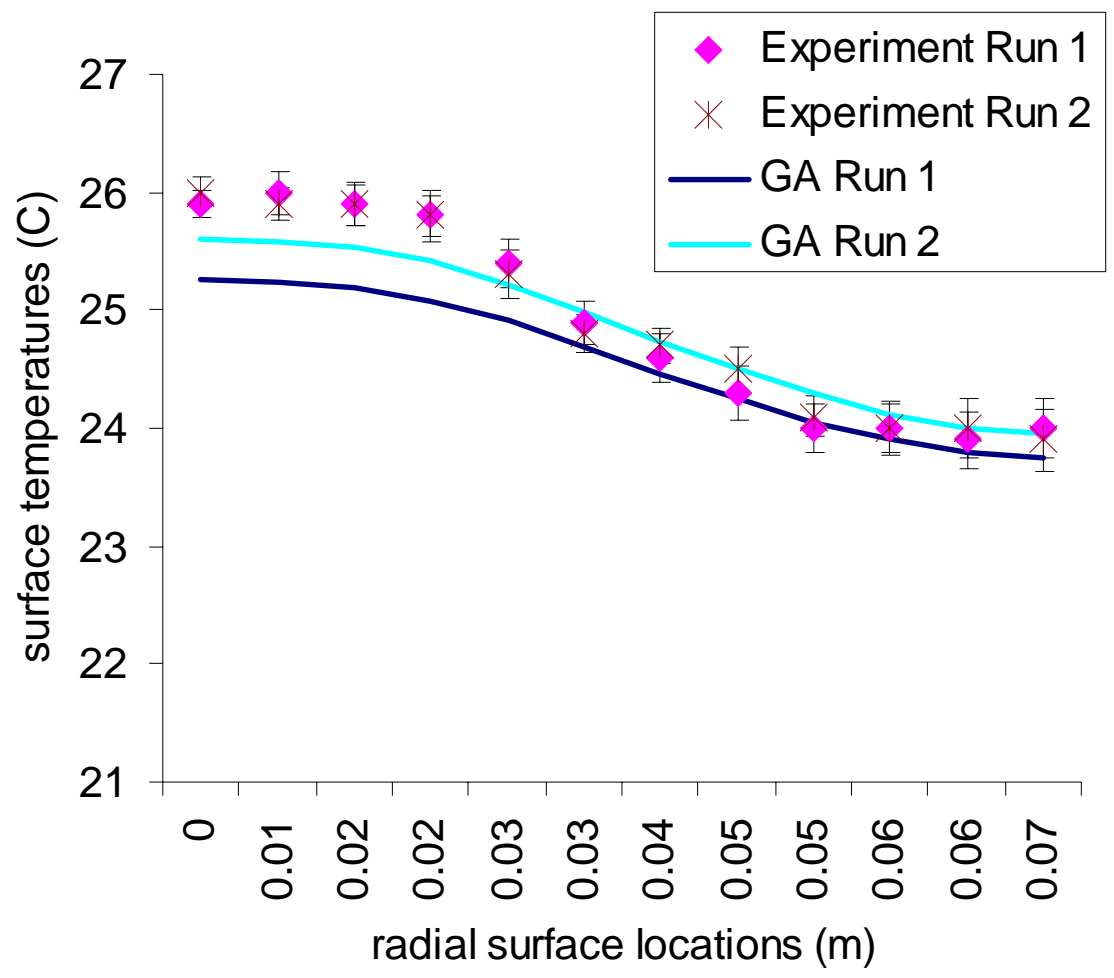

Fig. 6.8 Result of Experiment B1 showing experimental data and predicted temperature profiles

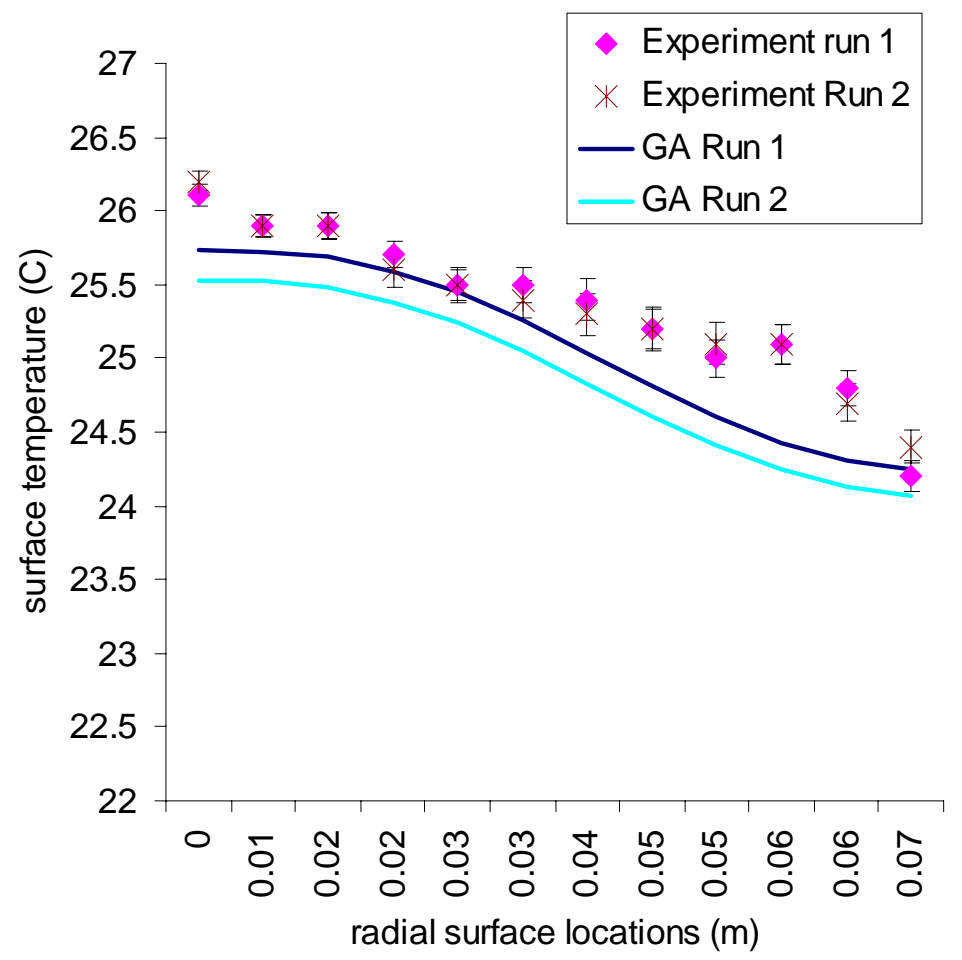

Fig. 6.9 Result of Experiment B2 showing experimental data and predicted temperature profiles 


\subsection{Sensitivity Analysis}

In order to study the effect of uncertainty in the convection coefficient and thermal conductivity on the model output, two experimental cases, A1 and B2, were analyzed further. For experiment A1, since it was assumed that the thermal conductivity is known, only the effects of variation in convection coefficient were examined. Fig. 6.10 shows the experimental data and the direct model solution based on nominal value of convection coefficient as well as its upper and lower confidence limits.

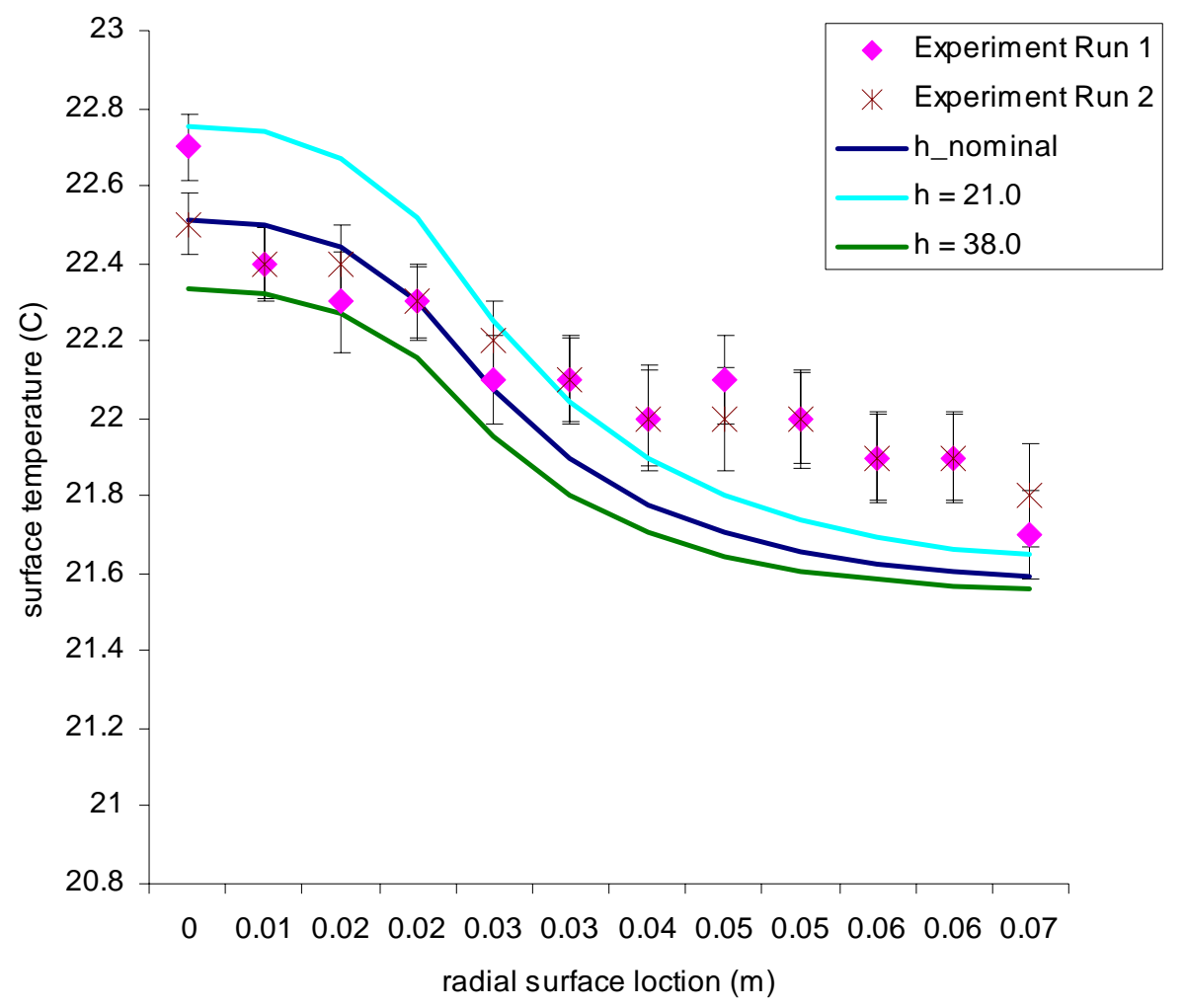

Fig. 6.10 The effect of convection coefficient on model output for experiment A1

For case B2, the effects of changing the convection coefficient and thermal conductivity within their confidence intervals are examined. The results are shown in Fig. 6.11 and Fig. 6.12 respectively.

It can be observed that the convection coefficient value has the effect of scaling the temperature profile. A lower value of convection coefficient causes the temperature profile to move up, while a higher value makes it move down. This behavior is expected 
since lower convection leads to less efficient dissipation of heat from the system. Also, since the cylinder is insulated on all sides and top surface convection is the only means of heat removal from the system, the effect of changing convection coefficient is much more pronounced than it would be otherwise.

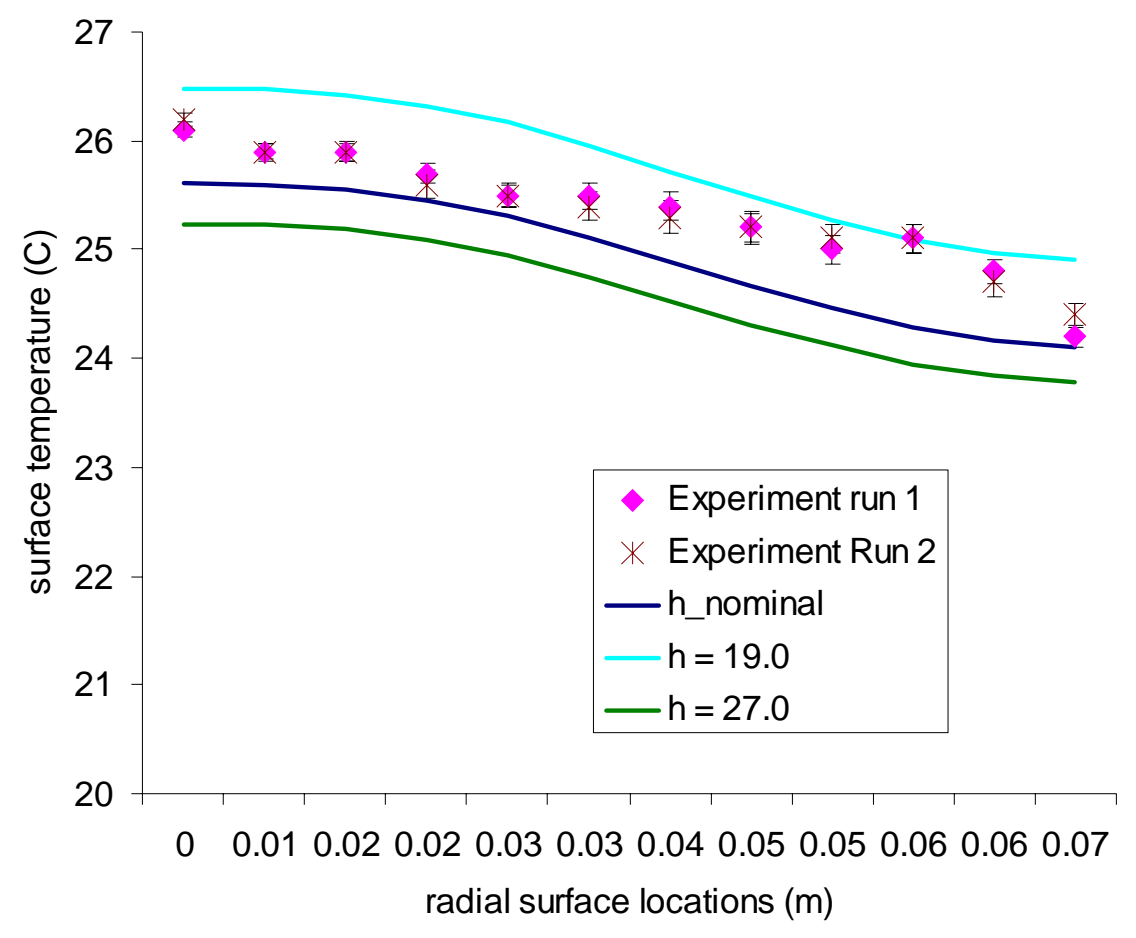

Fig. 6.11 The effect of convection coefficient on model output for experiment B2

Fig. 6.12 shows that the effect of thermal conductivity is primarily to change the shape of the temperature profile. A higher thermal conductivity leads to a more leveled temperature profile, reducing the temperature differential observed on the surface. This is the expected behavior since a higher thermal conductivity means better diffusion of heat from the center of the cylinder to its periphery. 


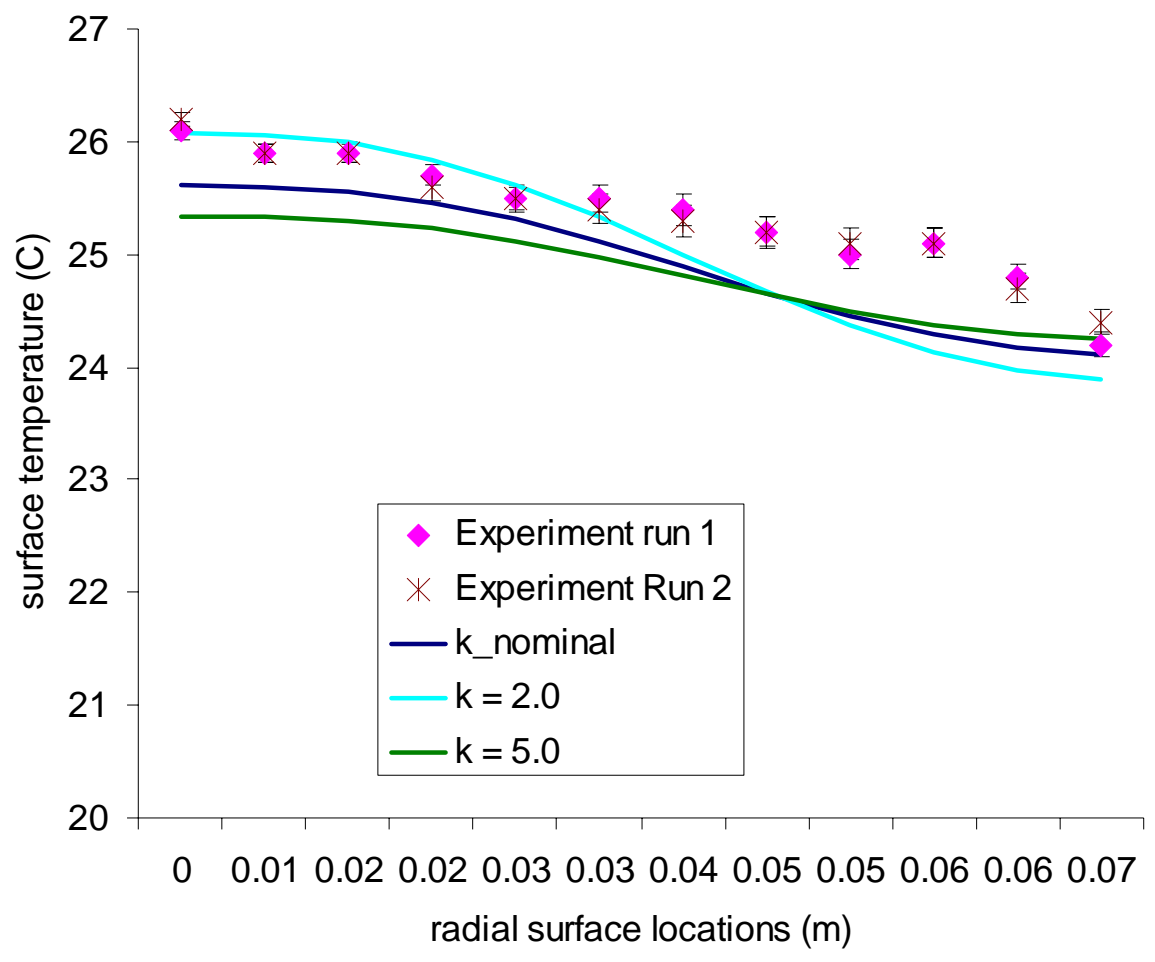

Fig. 6.12 The effect of thermal conductivity on model output for experiment B2 


\section{Chapter 7 Conclusions and Recommendations}

\subsection{Conclusions}

The overall objectives outlined in Section 1.1 were achieved through this work. The following list presents the main conclusions that can be drawn from this research. These conclusions cover the areas of thermal imaging, the biological aspects of development of tumors, and the parameter estimation program.

1. A review of thermal imaging reveals that the development of high-resolution digital infrared imaging, computerized image storage and comparison, and sophisticated technologies for image enhancement and analysis, offer potential in the use of thermography as an adjunct technique of detecting cancer. It still remains an adjunct technique since it cannot detect microcalcifications in the breast that can be seen only by mammography.

2. Using sensitivity analysis, it was shown that surface temperature exhibits a very low sensitivity to the location of the tumor. This is because the variations in surface temperature caused by a tumor are extremely small, especially if the tumor is embedded deep below the skin. This makes gradient-based estimation methods unstable and difficult to use. A genetic algorithm was found to be a robust non-gradient based optimization technique to use in this case.

3. For the cases studied, results from simulations and experiments show that it is possible to determine the location and heat generation rate of a phantom heat source using surface temperature measurements. 


\subsection{Recommendations}

This research represents a significant step forward in the early diagnostics of tumors using thermal imaging, by completing a clinically applicable algorithm that can be used to recognize tumors and pinpoint their locations. However, several areas deserve further attention but were beyond the scope of this work. The overall recommendations for advancement of the thermal tumor detection research are explained in the paragraphs below.

1. A simplified two-dimensional model was used in this research with a constant size planar heat source representing the metabolic heat generation in a tumor. This simplified study validates the idea of using thermal imaging as a means of detecting an embedded heat source. The model can further be extended for use with more realistic three-dimensional complex geometries and a spherical heat source representing the tumor. The extended study can provide further insight into whether it would be possible to determine both the size and location of the tumor, and how accurately the parameters can be predicted in a realistic situation.

2. Although the Pennes bio-heat equation appears to give adequate results in numerous applications, a precise description of heat transfer in living tissue is a challenging task. The flow of thermal energy in a living tissue is extremely difficult to model both due to disordered geometry in the tissue structure and a large number of interdependent concurrent processes in the tissue. In order for the estimation procedure to be highly accurate, the heat transfer equation used to model the direct problem should closely mirror the underlying physical processes in the tissue. It would be useful to study the limitations of bio-heat transfer equation and seek alternatives.

3. Although it was found in this study that the effect of metabolic heat liberation dominates the effect of increased blood circulation in tumors, the exact effects of 
increased blood circulation on the temperature distributions merit further investigation. Any such investigation would have to take into account the density of veins and capillaries around a tumor as well as their cross-sectional areas.

4. Reliable data on in-vivo thermal properties of the tissue and tumor is limited. Accurate values of properties like blood perfusion, thermal conductivity and specific heat are necessary in order for the parameter estimation program to be effective. There are currently no standardized methods to measure these values. Data should be collected over a wide range of temperatures and for different periods in the growth of the tumor.

5. The estimation algorithm should be tested on a variety of biological tissues in a controlled environment. Although the phantom experiments conducted using agar show the validity of the parameter estimation technique, further in-vitro experiments should be performed that mimic the biological conditions more closely. These experiments could include bringing in tumors or biological tissue to the test tube. This can then be followed by testing on animals and humans, both healthy and those known to have a tumor. Since thermal imaging is a completely non-invasive procedure, the use of animal or human subjects does not pose any ethical concerns. The information obtained from these tests can be incorporated into the mathematical model developed in this research.

6. The genetic algorithm used as the parameter estimation method in this research uses mutation and simple crossover operators. Several natural operators and phenomenon such as inversion, deletion, translocation, etc. remain to be explored in order to improve upon the robustness of simple GAs.

7. Skin temperatures are highly sensitive to the heat transfer coefficient. Although the imaging procedure is carried out in a thermally controlled environment, obtaining accurate estimates for the heat transfer coefficient during the procedure 
remains a challenging task. The heat transfer coefficient should take into account the effects of convection, radiation and evaporation.

8. This research can be extended further by considering the use of techniques such as skin surface cooling and measuring the transient response. Although a transient analysis would be computationally more expensive than the steady state analysis presented in this research, it may enhance the signature of the tumor on the surface and may possibly help alleviate the low sensitivity problem typically encountered in medical thermal imaging.

This research provides a foundation for the recommendations mentioned above. The described theory and experiments validate the idea of combining thermal imaging data, biological properties of tumors, and parameter estimation techniques, in order to non-invasively predict the presence of a tumor and pinpoint its location. The key to cancer survival is its earliest possible detection. This work contributes to increasing the valuable knowledge in the field of early cancer detection using non-invasive technique of thermal imaging. 


\section{Bibliography}

Adams F., 1939, “The Genuine Works on Hippocrates,” Williams and Wilkins, Baltimore.

Albritton E. C., 1954, Standard Values in Nutrition and Medicine, W.B. Saunders Co.

Amalu, W.C., 2003, “A Review of Breast Thermography,” International Academy of Clinical Thermology.

Barrett A.H., Myers P.C., 1975, “Subcutaneous temperatures: a method of noninvasive sensing,” Science, Vol. 190, pp. 669-71.

Barrett A.H., Myers P.C., and Sadowsky N.L., 1980, "Microwave thermography in the detection of breast cancer,” AJR Am J Roentgenol, Vol. 34(2), pp. 365-8.

Booth J., Boyland E. and Cooling C., 1967, “The respiration of human liver tissue,” Biochemical Pharmacology, Vol. 16(4), pp. 721-724.

Bard Y., 1974, Nonlinear Parameter Estimation, Academic Press.

Beck, J. V. and Arnold, K. J., 1977, Parameter Estimation in Engineering and Science, John Wiley \& Sons.

Beck J.V., Blackwell B. and St. Clair C.R., 1985, Inverse Heat Conduction: Ill-posed Problems, John Wiley and Sons.

Box, G. E. P. and Kanemasu, H., 1972, “Topics in Model Building, Part II, on Nonlinear Least Squares," Tech. Rep. 321, University of Wisconsin, Dpartment of Statistics, Madison,Wisconsin. 
Chen, M.M., and Holmes, K.R., 1980, "Microvascular contributions in tissue heat Transfer,” Ann. N.Y. Acad. Sci., 335, pp.137-154.

Davalos R. V. and Rubinsky B., 1996, “An Evolutionary-Genetic Approach to Heat Transfer Analysis,” Journal of Heat Transfer, Vol. 118, pp. 528-531.

Davis, L., 1991, Handbook of Genetic Algorithms, Van Nostrand Reinhold.

Draper J.W. and Boag J.W., 1971, "Skin temperature distributions over veins and tumours,” Phys. Med. Biol., Vol. 16(4), pp. 645-54.

Doyle J. F., 1995, "Determining the Size and Location of a Crack in a Frame Structure,” Proc. of the 7th Inverse Problems in Engineering Seminar, OSU.

Emery, A. and Fadale, T., 1996, "Design of Experiments Using Uncertainty Information,” ASME Journal of Heat Transfer, Vol. 118, pp. 532-538.

Fitzpatrick J. M., Grefenstette J. J., and Van Gucht D., 1984, “Image Registration by Genetic Search,” Proceedings of IEEE Southeast Conference, pp. 460-464.

Folkman J., 1976, “The Vascularization of Tumors,” Scientific American, Vol. 234(5), pp. 58-64, 70-3.

Fujimasa I., Sakurai Y., and Atsumi K., 1973, Medical Thermography, University of Tokyo Press.

Furuya, H. and Haftka, R. T., 1993, "Genetic Algorithms for Placing Actuators on Space Structures," Proc. of the Fifth International Conf. on Genetic Algorithms, UrbanaChampaign, IL, pp. 536-542. 
Gershen-Cohen J., Haberman J., and Brueschke E.E., 1965, “Medical thermography: A summary of current status,” Radiol Clin North Am 3:403-431.

Goldberg D.E., 1989, Genetic Algorithms in Search, Optimization and Machine Learning, Addison-Wesley.

Garcia S., 1999, "Experimental Design Optimization and Thermophysical Parameter Estimation of Composite Materials Using Genetic Algorithms," Dissertation, Virginia Polytechnic Institute and State University.

Haberman J., 1968, “The present status of mammary thermography,” In: Ca - A Cancer Journal for Clinicians 18: 314-321.

Haberman J., 1986, “An overview of breast thermography in the United States,” In: Margaret Abernathy, Sumio Uematsu (Eds): Medical Thermography. American Academy of Thermology, Washington, pp.218-223.

Haftka R. T. and Gurdal Z., 1992, Elements of Structural Optimization, Kluwer Academic Pub.

Holland, J.H., 1975, Adaptation in Natural and Artificial Systems, University of Michigan Press.

Hata T., Takahashi H., Watanabe K., Takahashi M., Taguchi K., Itoh T. and Todo S., 2004, "Magnetic resonance imaging for preoperative evaluation of breast cancer: a comparative study with mammography and ultrasonography,” Journal of the American College of Surgeons, Vol. 198(2), pp. 190-197.

Incropera, F. P. and Dewitt, D. P., 2001, Fundamentals of Heat and Mass Transfer, John Wiley \& Sons. 
Lawson R., 1956, "Implications of Surface Temperatures in the Diagnosis of Breast Cancer,” Can Med Assoc J 75: 309-310.

Lawson R.N. and Chughtai M.S., 1963, "Breast cancer and body temperatures," Can Med Assoc. J 88:68-70.

Lee C.H. and Weinreb J.C., 2004, "The use of magnetic resonance imaging in breast cancer screening,” Journal of the American College of Radiology, Vol. 1(3), pp. 176-182.

Mitchell M., 1996, An Introduction to Genetic Algorithms, Complex Adaptative System Series, Bradford Pub.

Mottley J.G., 1995, “Ultrasonic Imaging,” The Biomedical Engineering Handbook, CRC Press.

National Institutes of Health, 1977, "Breast Cancer Screening," NIH Consensus Statement Online, Sep 14-16; 1(1): 5-8.

Nilsson S.K., Gustafsson S.E., and Torell L.M., 1980, "Skin temperature over a heat source: experimental studies and theoretical calculations,” NY Academy of Sciences, Vol 335, pp. 416-428.

Ng, E. Y. K., and Sudershan, N. M., 2001, “An improved 3D direct numerical modelling and thermal analysis of a female breast with tumour”, International Journal of Engineering in Medicine, Proceedings of the IMechE, Part H, 215(1), 25 - 37.

Patankar S.V., 1980, Numerical Heat Transfer and Fluid Flow, Hemisphere Pub.

Peaceman D.W. and Rachford H.H., 1955, "The numerical solution of parabolic and elliptic differential equations,” J. Soc. Ind. Appl. Math., vol. 3, pp. $28-41$. 
Pennes, H., 1948, “Analysis of Tissue and Arteriole Blood Temperatures in the Resting Forearm,” Journal of Applied Physiology, Vol. 1, pp. 93-122.

Romrell, Lynn J., Bland and Kirby I., "Anatomy of Breast, Axilla, Chest Wall, And Related Metastatic Sites in the Breast: A Comprehensive Management of Benign and Malignant Diseases,” Philadelphia, Saunders. pp 22, 1991.

Roskelley, R. C., Mayer, N., Horwitt, B. N. and Salter, W. T., 1943, "Studies in Cancer,” Journal of Clinical Investigation, Vol. 22, pp. 743-51.

Rubinsky B. and Davalos R.V., 1998, "The Use of Evolutionary-Genetic Analogy in Numerical Analysis,” Commun. Numer. Meth. Eng., vol. 14, pp. 151-160.

Schaefer D.J., 2003, “Design of Magnetic Resonance Systems,” Standard Handbook of Biomedical Engineering and Design, McGraw-Hill Pub.

Sudarshan N.M., Ng E.Y.K. and Teh, S.I., 1995, "Surface Temperature Distribution of a Breast With and Without Tumor," Computer Methods in Biomechanics and Biomedical Engineering, Vol. 2, pp. 187-199.

Steketee J., 1973, “Spectral emissivity of skin and pericardium,” Phys. Med. Biol., Vol. 18(5), pp. 668-94.

Thomenius K.E., 2003, "Instrumentation Design for Ultrasonic Imaging," Standard Handbook of Biomedical Engineering and Design, McGraw-Hill Pub.

Tsourkas P. and Rubinsky B., 2003, "Evolutionary-Genetic Algorithm for Solving 2-D Steady- State Conduction Problems,” Numerical Heat Transfer Part B, Vol. 43(2), pp. 99117. 
Vick B., 2003, Finite Difference Method, Department of Mechanical Engineering, Virginia Tech.

Warren R., 2001, "Screening women at high risk of breast cancer on the basis of evidence,” European Journal of Radiology, Vol. 39(1), pp. 50-59.

Werner J. and Buse M., 1988, “Temperature profiles with respect to inhomogeneity and geometry of the human body,” Journal of Applied Physiology, Vol. 65(3), pp. 1110-8.

Yaffe M.J., 1995, “Mammography,” The Biomedical Engineering Handbook, CRC Press. 


\section{Appendix A: Finite Difference Code}

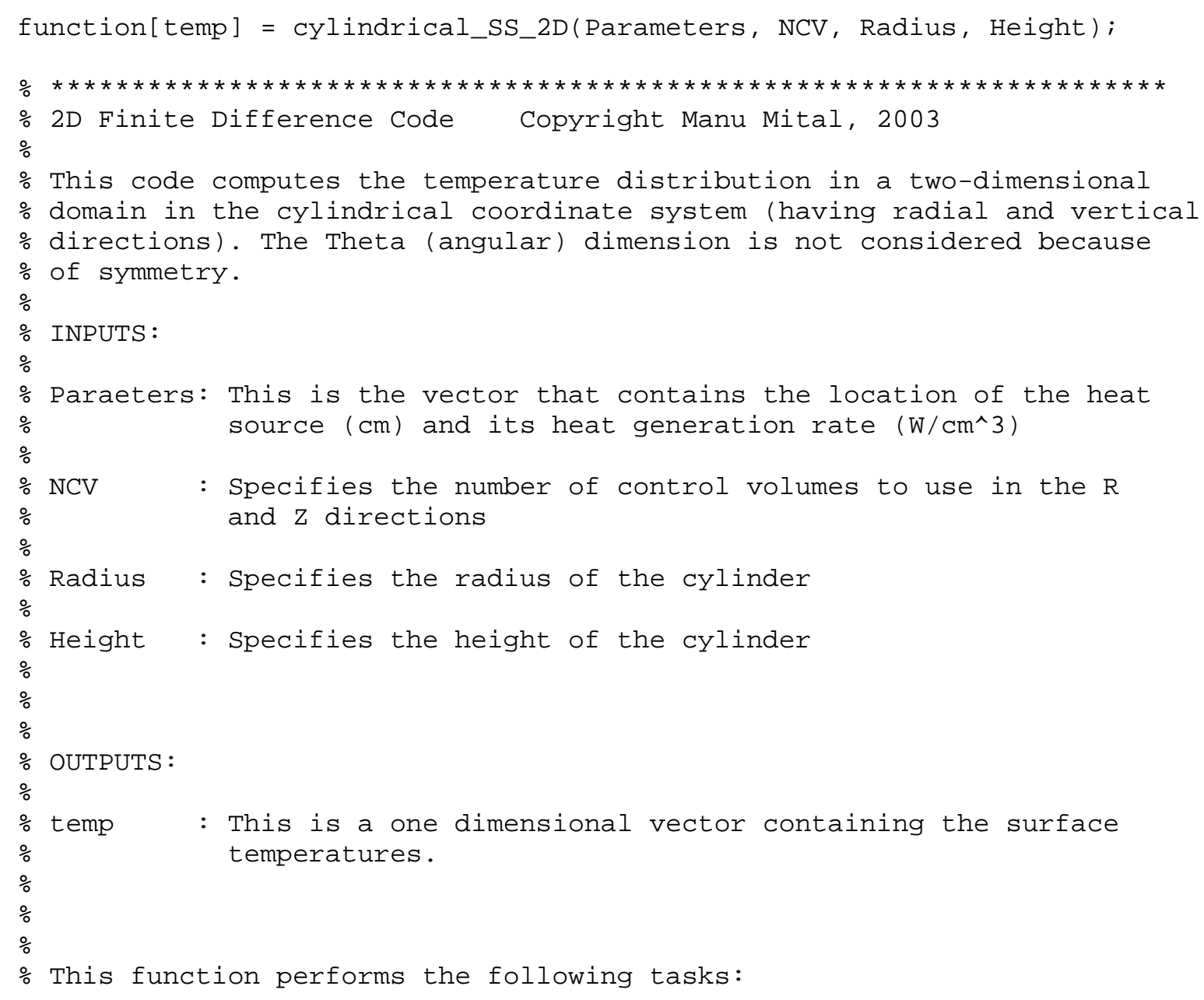


\% 1. takes in the dimensions of domain and the parameters

$\%$ 2. discretizes space based on number of control volumes specified

$\% \quad 3$. Applies specified boundary conditions

\% 4. Calls ADIT solver

$\%$ 5. If required, it can plot the surface temperature vector

$\% \quad$ versus the radial location.

$\mathrm{tr}=2$

\%\%\%\%\%\%\%\%\%\%\%\%\%\%\%\%\%\%\%\%\%\%\%\%\%\% INPUT SECTIONS BEGIN \%\%\%\%\%\%\%\%\%\%\%

\%NR is the number of non-zero control volumes into which the body is divided

$\%$ along the path of heat transfer in the $\mathrm{R}$ direction

$\mathrm{R}=$ Radius; $\quad \mathrm{NR}=\mathrm{NCV} ; \quad \%$ Total control volumes $=\mathrm{NR}+2$

$\% N Z$ is the number of non-zero control volumes into which the body is divided

$\%$ along the path of heat transfer in the $\mathrm{Z}$ direction

$\mathrm{H}=$ Height; $\quad \mathrm{NH}=\mathrm{NCV} ; \quad$ \% Total control volumes $=\mathrm{NZ}+2$

$\%$ set the grid size by specifying deltaR and deltaz

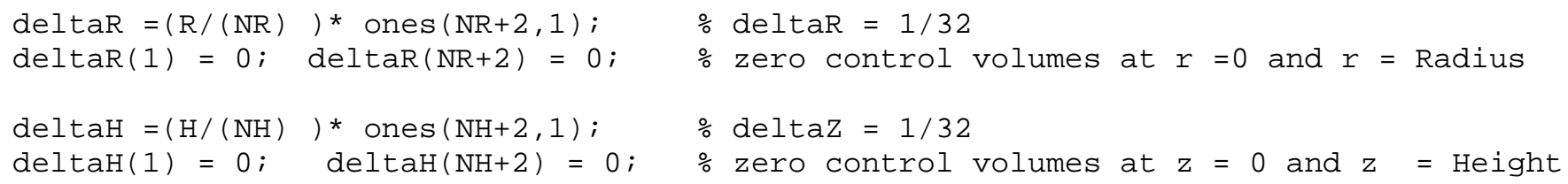


$\%$ What kind of boundary conditions do you need?

$\% 1$ indicates that the temperature has been specified as boundary conditions

$\% 2$ indicates that heat flux and/or convection specified at the boundaries.

$\mathrm{BCr} \odot=2 ; \quad \mathrm{BCrR}=2 ; \quad \mathrm{BCz} \odot=2 ; \quad \mathrm{BCzH}=2 ;$

\% Specify the variables depending on the boundary conditions you need.

$\%$ These properties at the boundaries are specified as being constants along a boundary.

$\%$ However, if they vary, then specify them as vectors.

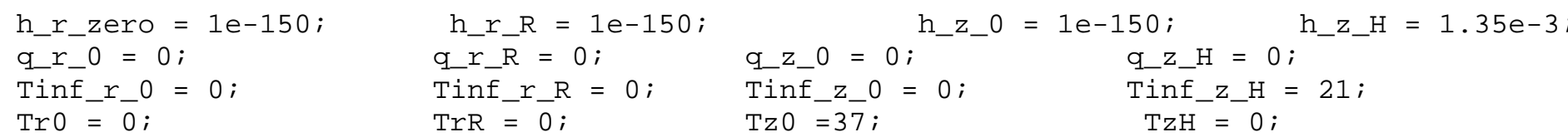

\% Create the matrices SC and SP

$\mathrm{SC}=(.7 \mathrm{e}-3) *$ ones $(\mathrm{NR}+2, \mathrm{NH}+2)$;

$\mathrm{SP}=0^{*}(2.4 \mathrm{e}-3) *$ ones $(\mathrm{NR}+2, \mathrm{NH}+2)$;

for $i=1: \operatorname{tr} \operatorname{SC}(i$, Parameters $(1))=$ Parameters $(2)$; end

$\%$ The thermal conductivity matrix

$\mathrm{k}=(4.8 \mathrm{e}-3) *$ ones $(\mathrm{NR}+2, \mathrm{NH}+2) ;$

\% \%\%\%\%\%\%\%\%\%\%\%\%\%\%\%\%\%\%\%\%\%\%\%\%\%\%\%\%\%\%\%\%\%\%\%\%\% END OF THE INPUT SECTION \%\%\%\%\%\%\%\%\%\% 
\%\%\%\%\%\%\%\%\%\%\%\%\%\%\%\%\%\%\%\%\%\%\%\%\%\%\%\%\%\%\%\%\%\% PROCESSOR \%\%\%\%\%\%\%\%\%\%\%\%\%\%\%\%\%\%\%\%\%\%\%\%\%\%\% $\%$ Define the temperature vector that has elements from 1 to NR+2 in the R-direction.

$\%$ It has elements from 1 to $\mathrm{NH}+2$ in the $\mathrm{Z}$ direction. We initialize the temperature $\%$ vector with zeros

$\mathrm{T}=\operatorname{zeros}(\mathrm{NR}+2, \mathrm{NH}+2) ;$

$\%$ Define the $b$ vector and fill in its components

$\mathrm{B}=\operatorname{zeros}(\mathrm{NR}+2, \mathrm{NH}+2)$;

for $i=1: N R+2$

for $\mathrm{j}=1: \mathrm{NH}+2$ end $B(i, j)=S C(i, j) * R \_L O C(i) * \operatorname{deltaR}(i) * \operatorname{deltaH}(j)$;

end

$\operatorname{aij}=\operatorname{zeros}(\mathrm{NR}+2, \mathrm{NH}+2)$;

$\%$ The following formulation is valid for $i=2$ to $i=N X+1$ and from $j=2$ to $j=N Y+1$ aeij $=\operatorname{zeros}(\mathrm{NR}+2, \mathrm{NH}+2) ; \quad \operatorname{anij}=\operatorname{zeros}(\mathrm{NR}+2, \mathrm{NH}+2)$;

awij $=\operatorname{zeros}(\mathrm{NR}+2, \mathrm{NH}+2) ; \quad \operatorname{asij}=\operatorname{zeros}(\mathrm{NR}+2, \mathrm{NH}+2) ;$

$\%$ awij and aeij matrices represent the east and the west side

for $i=1: N R+1$

for $\mathrm{j}=1: \mathrm{NH}+2$

$\operatorname{aeij}(i, j)=\operatorname{deltaH}(j) *(2 * k(i, j) * k(i+1, j)) /[(\operatorname{deltaR}(i) * k(i+1, j)+\operatorname{deltaR}(i+1) * k(i, j))] ;$

end

end

for $\mathrm{j}=1: \mathrm{NH}+2$ $\operatorname{awij}(1, j)=0 ; \quad \%$ The first set of control volumes have no west neighbour end $\operatorname{aeij}(N R+2, j)=0 ; \quad \%$ The last set of control volumes have no east neighbour 


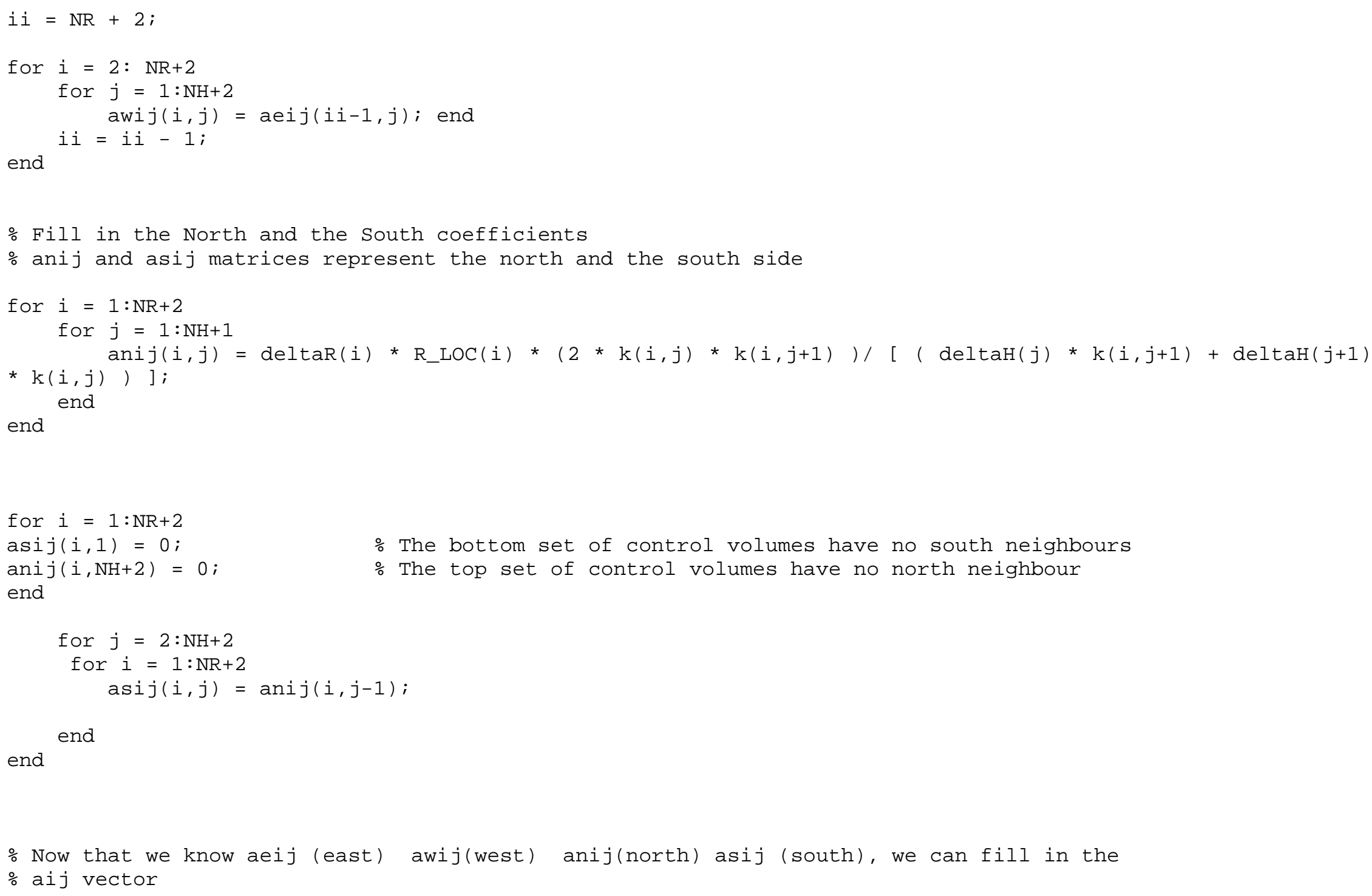


\% Boundary conditions at $\mathrm{z}=\mathrm{H} \quad$ These depend on the variable BCyL

if $\mathrm{BCZH}==1$

$$
\begin{aligned}
& \text { for } i=1: N R+2 \\
& \operatorname{asij}(i, \mathrm{NH}+2)=0 \text {; } \\
& \operatorname{aij}(\mathrm{i}, \mathrm{NH}+2)=1 \text {; } \\
& \mathrm{B}(\mathrm{i}, \mathrm{NH}+2)=\mathrm{TzH} \text {; } \\
& \text { end }
\end{aligned}
$$

elseif $\mathrm{BCzH}==2$

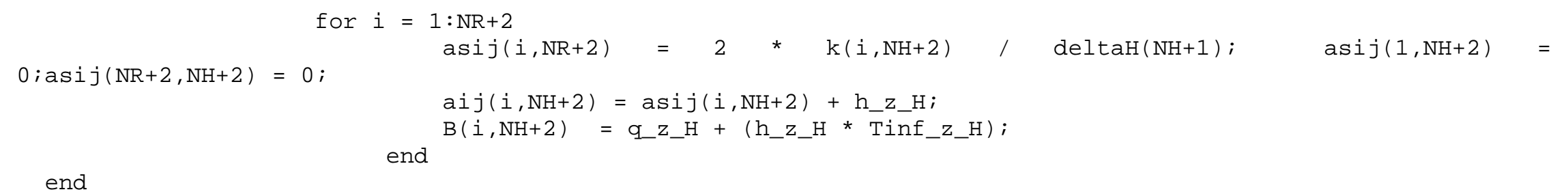

\%\%\%\%\%\%\%\%\%\%\%\%\%\%\% END OF BOUNDARY CONDITIONS \%\%\%\%\%\%\%\%\%\%\%\%\%\%\%\%\%\%\%\%\%\%\%\%\%\%\%\%\%\%\%\%\%\%\%

\% Finally we solve for the Temp

$\% \mathrm{~T}=$ GaussSeidel(aij, aeij, awij, anij, asij, $\mathrm{B}, \mathrm{T}, \mathrm{NR}+2, \mathrm{NH}+2)$

$\% \mathrm{t}=$ cputime;

$T=\operatorname{ADIT2}($ aij, aeij, awij, anij, asij, $\mathrm{B}, \mathrm{T}, \mathrm{NR}+2, \mathrm{NH}+2)$; 
\%cputime-t

temp $=\operatorname{zeros}(\mathrm{NCV}, 1)$;

$r=\operatorname{zeros}(\mathrm{NCV}, 1)$;

for $i=2: N R+1$

$r(i-1)=R \_L O C(i)$;

$\operatorname{temp}(i-1)=T(i, N H+2)$; end

\% Uncomment the following line if a plot of surface temperature distribution is required \%plot $\left(r\right.$, temp, '- $\left.x^{\prime}\right)$; hold on;

\%\%\%\%\%\%\%\%\%\%\%\%\%\%\%\%\%\%\%\%\%\%\%\%\%\%\%\%\%～RESULTS \%\%\%\%\%\%\%\%\%\%\%\%\%\%\%\%\%\%\%\%\%\%\%\%\%\%\%\%\%\%\%\% 


\section{Appendix B: Alternating-Direction Implicit Solver}

function $[U]=A D I T(A I J, A E I J, A W I J, A N I J, A S I J, B I J, T, X C V, Y C V)$

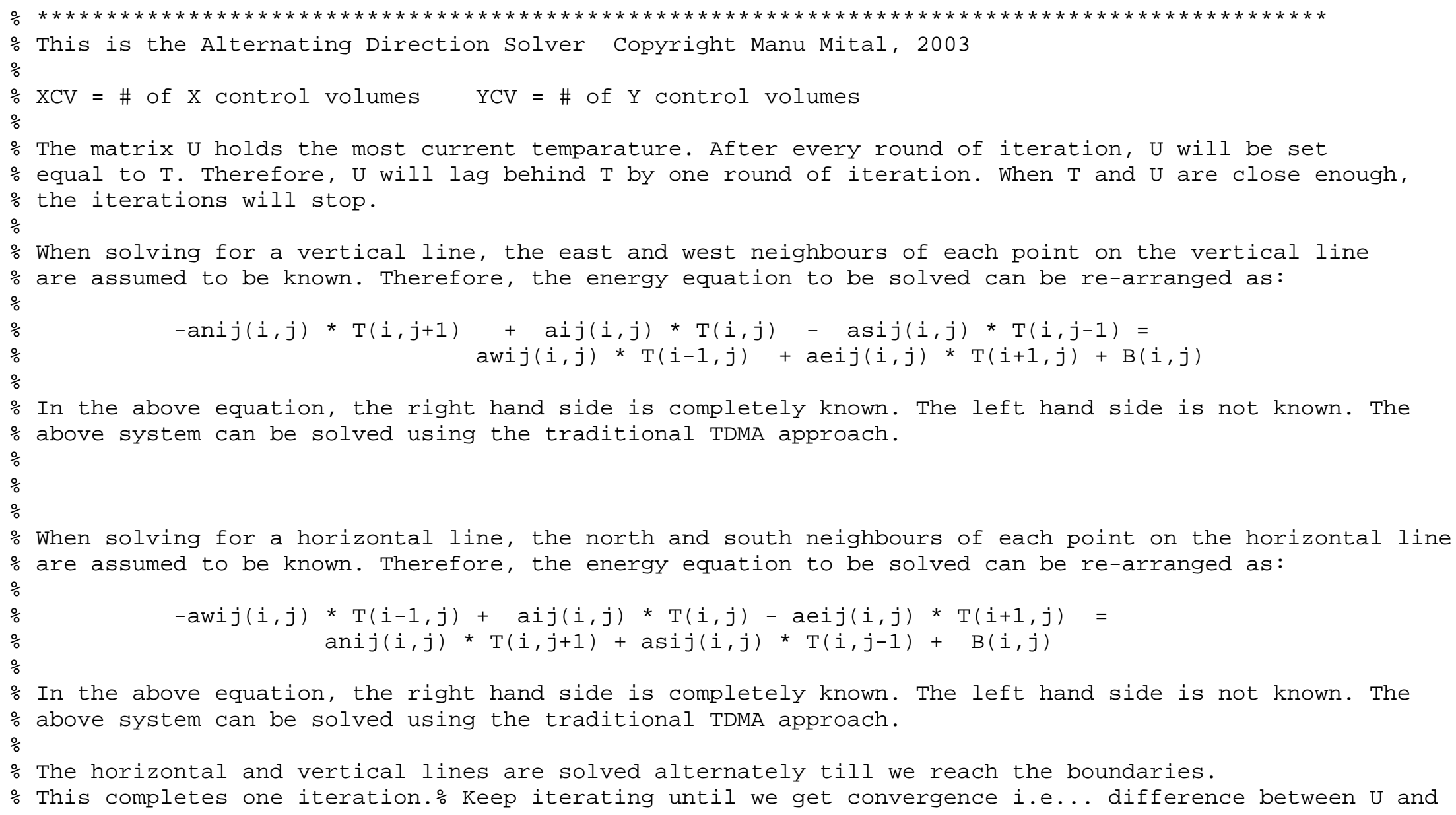




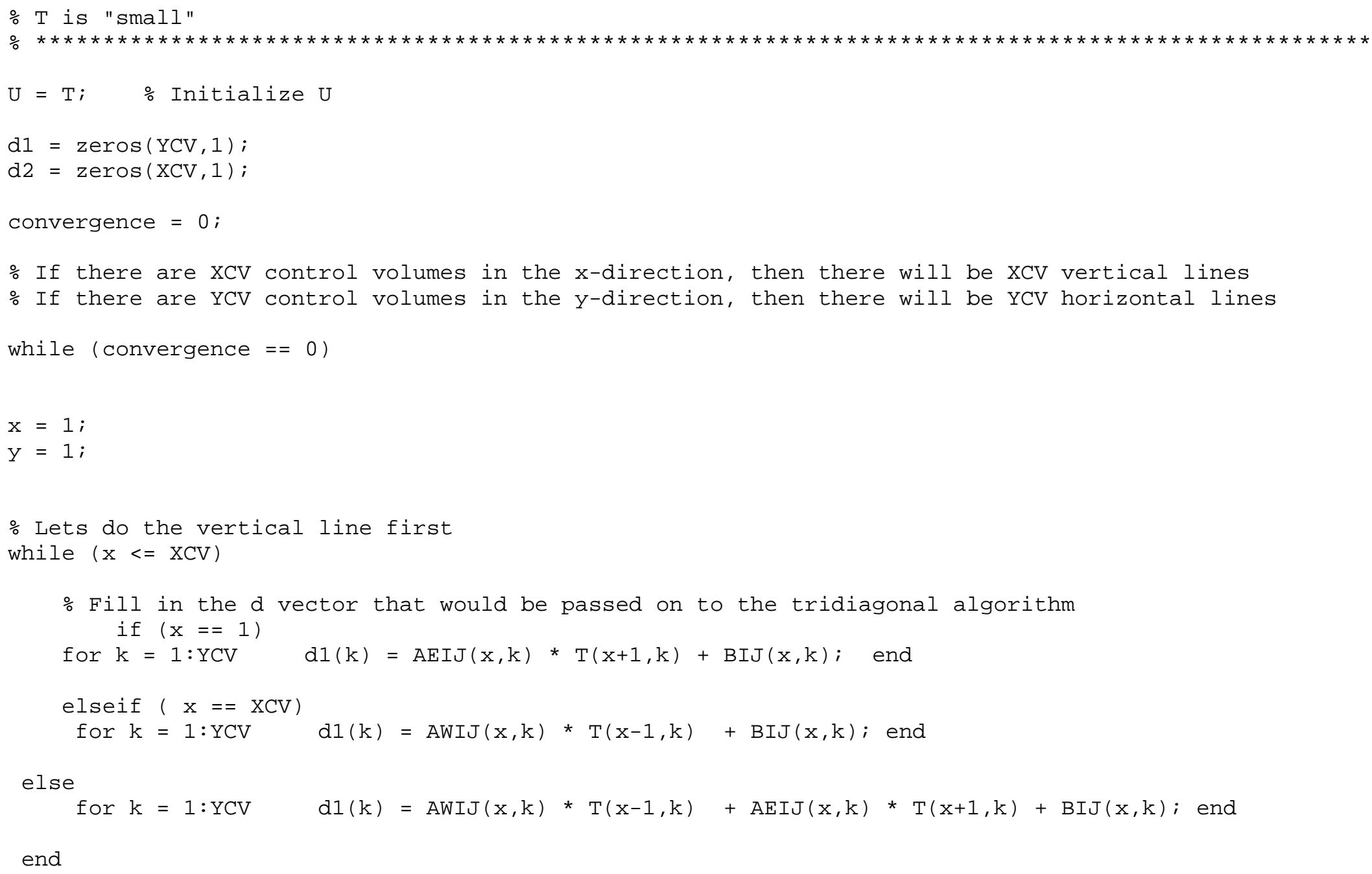




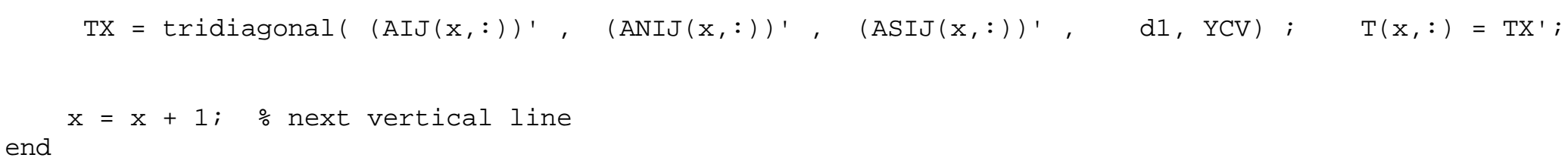


convergence $=1$;

$\%$ Check for convergence

for $m=1: 1: X C V$

for $\mathrm{n}=1: 1: \mathrm{YCV}$

if $\operatorname{abs}(\operatorname{abs}(T(m, n)-\operatorname{abs}(U(m, n)))>0.00001)$

convergence $=0 ;$ end

end

end \% stop the iterations

$\mathrm{U}=\mathrm{T}$;

end $\%$ end of convergence loop 


\section{Appendix C: Tridiagonal Matrix Algorithm (Thomas Algorithm)}

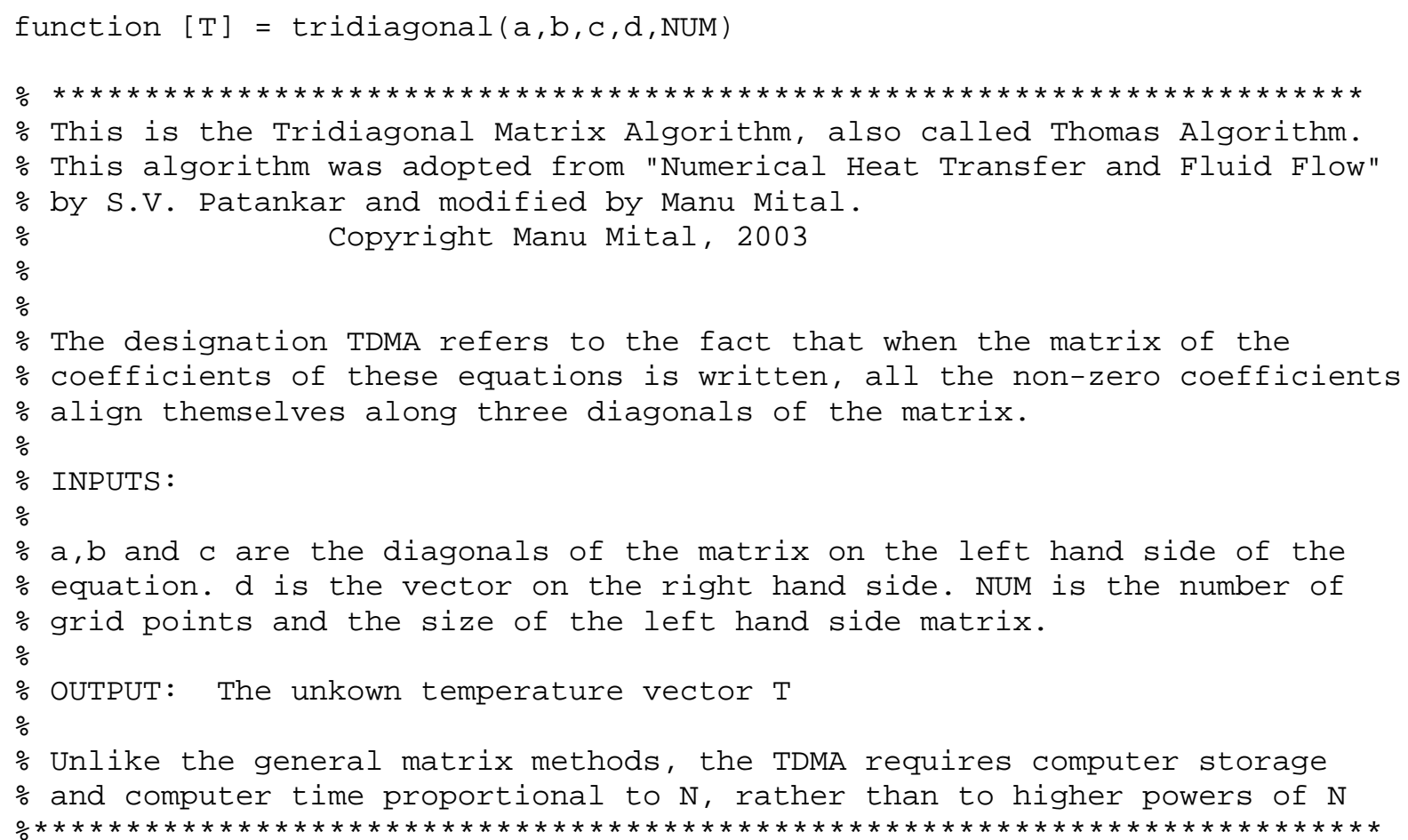




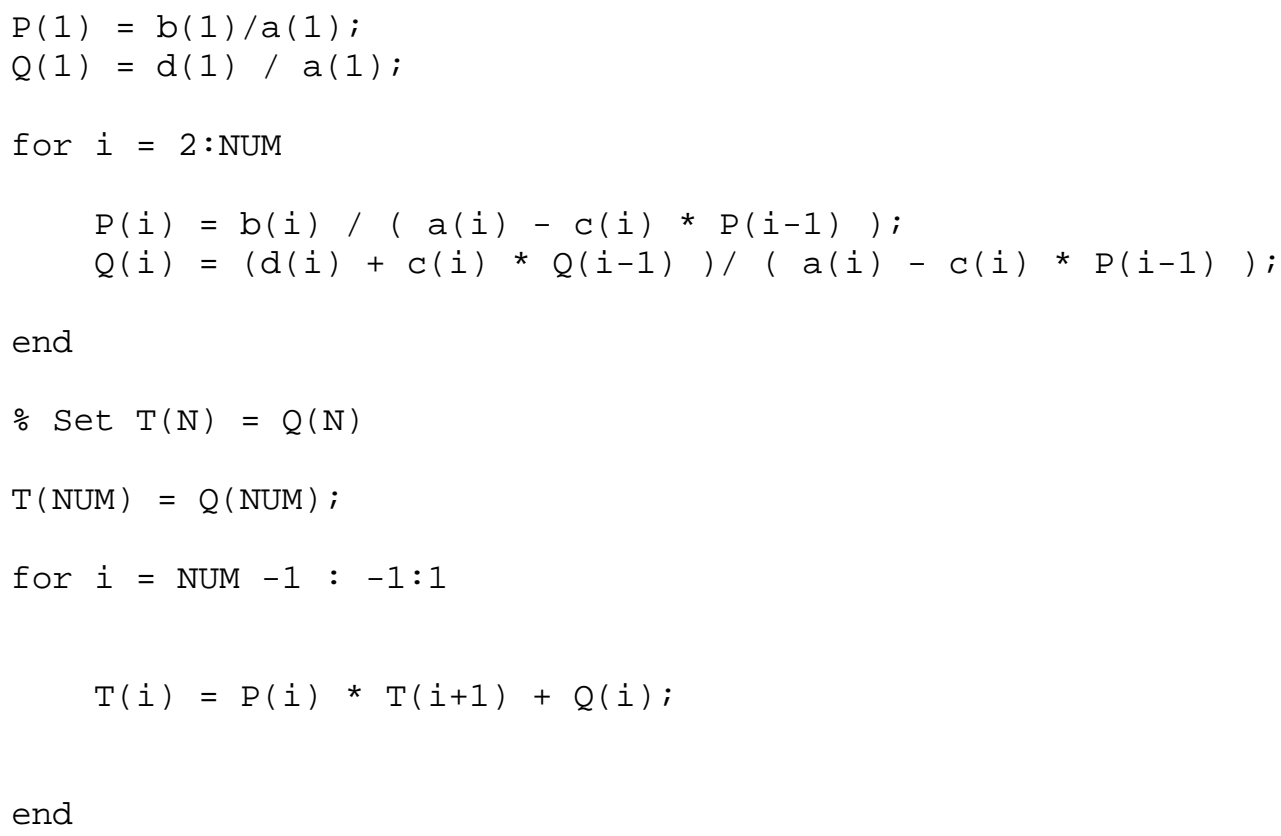




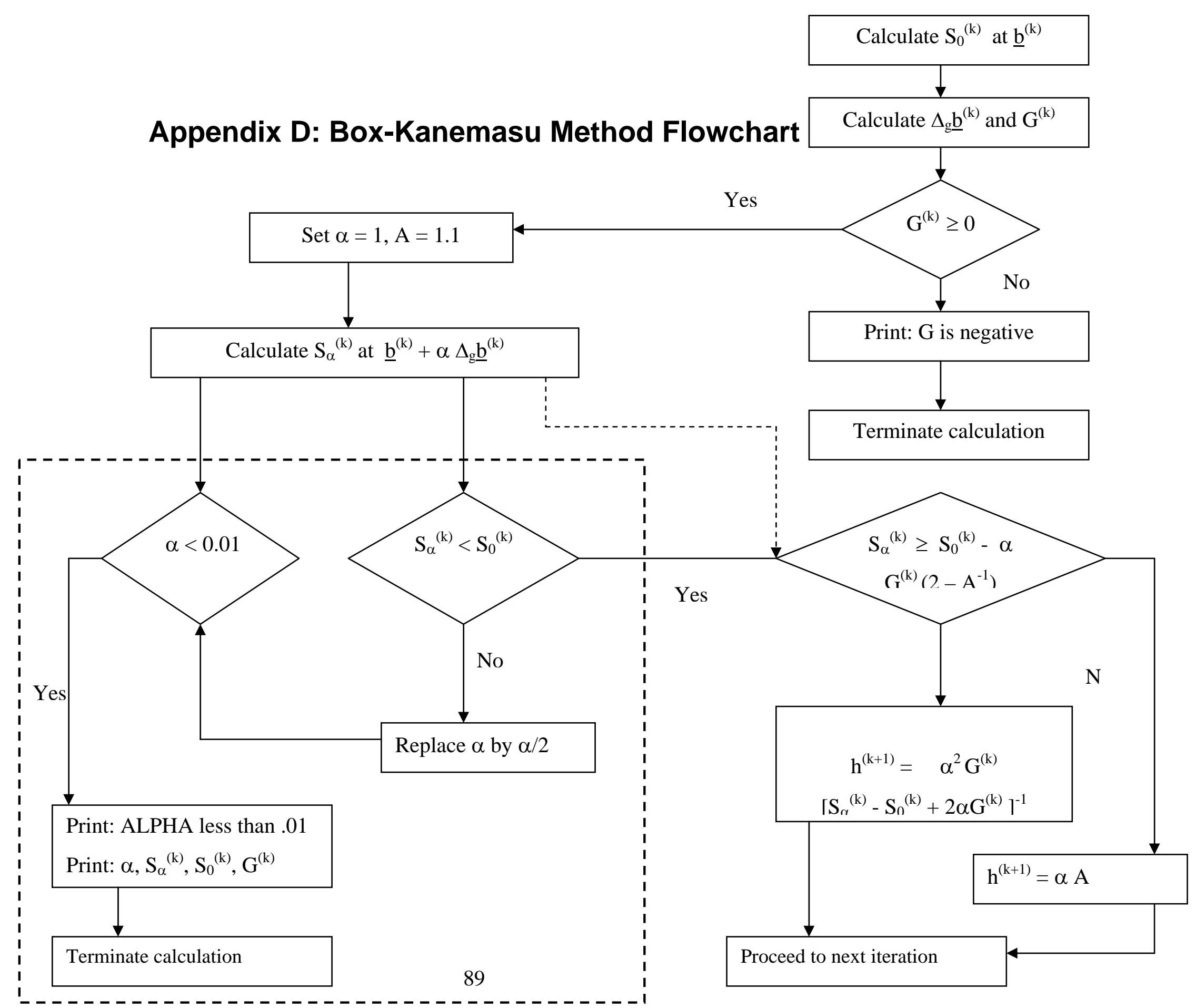




\section{Appendix E}

\section{E1 Box-Kanemasu Method Code for Parameter Estimation}

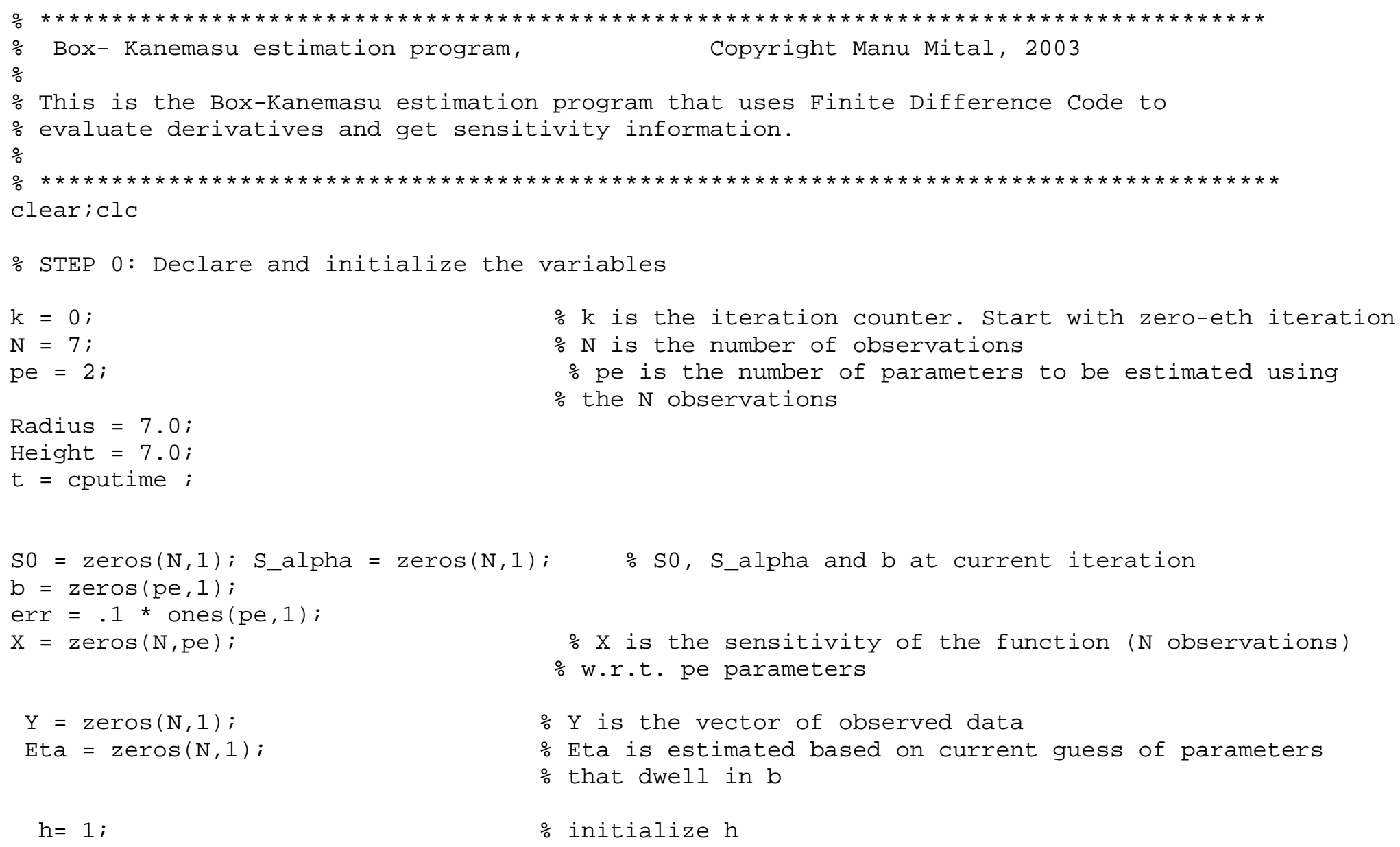


stop $=-1$

$\%$ Before starting the loop, we put an initial guess for the pe parameters in

$\%$ the $b$ vector. We also need to fill in data into $\mathrm{Y}$.

$\mathrm{b}=[4 ; 1 \mathrm{e}-3] ; \quad$ \%These are the initial guesses

b_direct $=[5 ; 1.4 \mathrm{e}-3]$;

$\mathrm{Y}=$ cylindrical_SS_2D(b_direct, N, Radius, Height ) ;

Eta = Cylindrical_SS_2D(b, N, Radius, Height );

while ( stop $==-1)$

\%\%\%\%\% STEP 1: Calculate S0 @ iteration k \%\%\%\%\%\%\%\%

SO $=(Y-E t a)^{\prime} *(Y-E t a) ;$

\%\%\%\% STEP 2: Update the value of sensitivity coefficient \%\%\%\%\%\%\%\%\%\%\%\%\%\%\%\%\%\%\%\%\%\% $X=\operatorname{updatex}(b, N$, Eta, Radius, Height $)$;

\%\%\%\%\% STEP 3: Calculate del_b and G \%\%\%\%\%\% \%\%\%\%\%\%\%\%\%\%\%\%\%\%\%\%\%\%\%\%\%\%\%\% Pinv $=\left(X^{\prime} * X\right) ; P=\operatorname{inv}(P i n v)$;

del_b $=P * \quad\left(X^{\prime} *(Y-E t a)\right)$

$G=$ del_b' * Pinv * del_b;

if $(G<0)$ stop $=1$; end 
\%\%\%\% STEP 4: Set the values of alpha and A \%\%\%\%\%\%\%\%\%\%\%\%\%\%\%\%\%\%\%\%\%\%

alpha $=\mathrm{h} ; \quad$ \% alpha is inititially set equal to 1

$\mathrm{A}=1.1 ;$

\%\%\% STEP 5: Update the value of $\mathrm{b}$ that holds the current guess of parameters $\% \% \%$

b_previous $=\mathrm{b} ; \quad \%$ This would always behind vector $\mathrm{b}$ by one iteration. When the vectors

$\% \mathrm{~b}$ and b_last come close enough, the iterations may be

$\%$ stopped

$b=b+($ alpha $) * d e l \_b ; \quad \%$ This is the updated value of $b$

\%\%\% STEP 6 : Stop iterations ?

if $($ (b - b_previous $)<=$ err $)$ stop = 1; end

\%\%\% STEP 7: Calculate S_alpha using b that was updated in last step \%\%\%\%\%\%\%\%\%\%\%\%\%\%\%\% Eta = Cylindrical_ss_2D(b, N, Radius, Height );

S_alpha $=(Y-E t a)^{\prime} *(Y-E t a)$;

S_check $=S 0-($ alpha $* G) *(2-(1 / A))$;

\%\%\% STEP 8 : Compare S_alpha with S_check and set the new value of $\mathrm{h} \% \% \% \% \% \% \% \% \% \% \% \% \% \%$ if $($ S_alpha $>=$ S_check $) h=\left(\left(\right.\right.$ alpha^2) * G) / (S_alpha - SO $\left.+2{ }^{*} a l p h a * G\right) ;$

else $h=$ alpha * $A$; end

end $\%$ end of the while loop

b \% print b

cputime- $t$ 


\section{UpdateX Function}

function $[x]=$ UpdateX(Pvector, $N$, eta, Radius, Height);

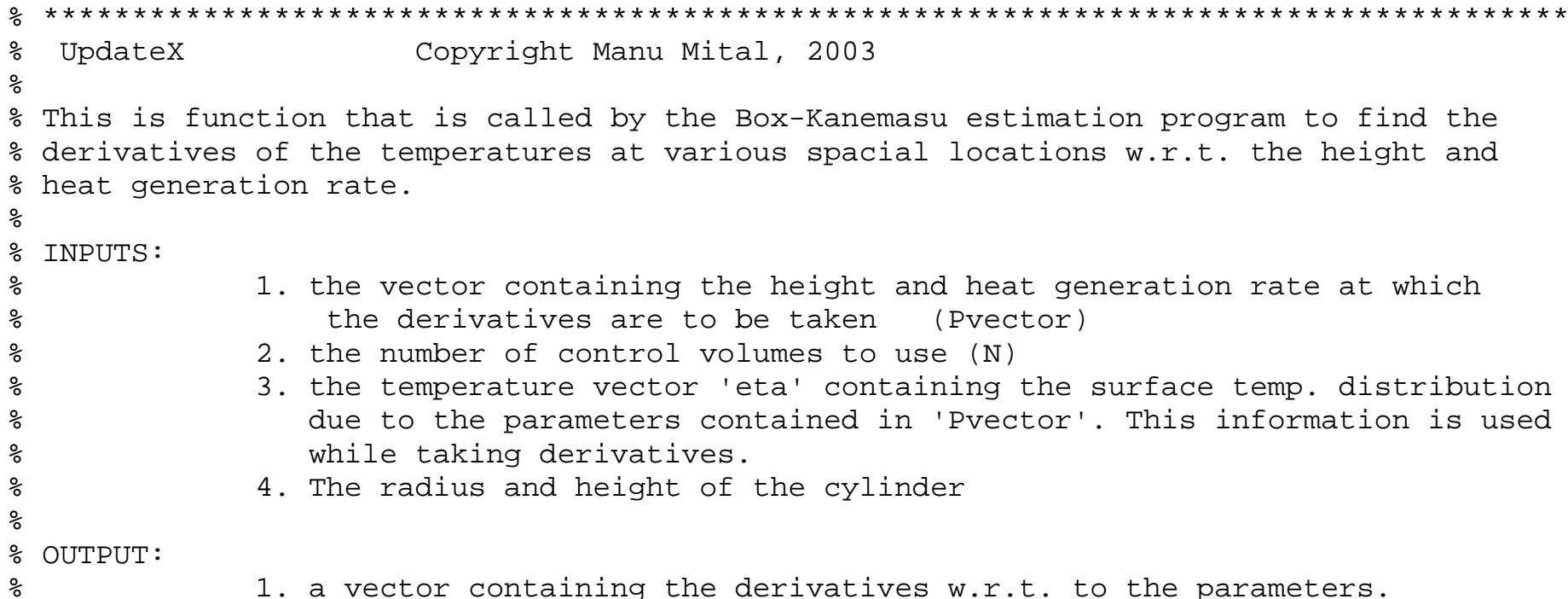

1. the vector containing the height and heat generation rate at which the derivatives are to be taken (Pvector)

2 . the number of control volumes to use $(\mathrm{N})$

3. the temperature vector 'eta' containing the surface temp. distribution due to the parameters contained in 'Pvector'. This information is used while taking derivatives.

4. The radius and height of the cylinder

num_param = size $($ Pvector, 1$) ;$

$\%$ Pvector $=$ [radius; height from base; heat generation rate]

$x=\operatorname{zeros}(N$, num_param $) ; \quad \%$ initialize the sensitivity coefficient vector

Pvector_modified = Pvector; Pvector_modified $(1)=$ Pvector_modified $(1)+1 ;$

F_delH = cylindrical_SS_2D(Pvector_modified, N,Radius, Height);

$x(:, 1)=($ F_delH - eta $)$;

Pvector_modified = Pvector; Pvector_modified $(2)=$ Pvector_modified $(2)+.1 \mathrm{e}-3$; 


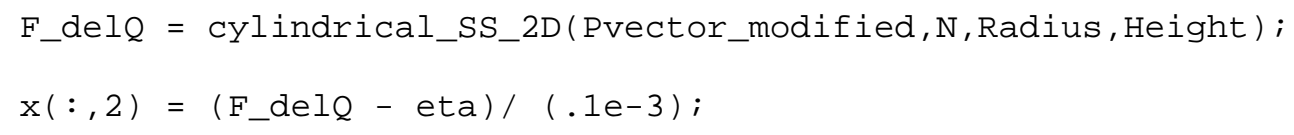

\section{E2 Box-Kanemasu Method Code for Estimating Convection Coefficient}

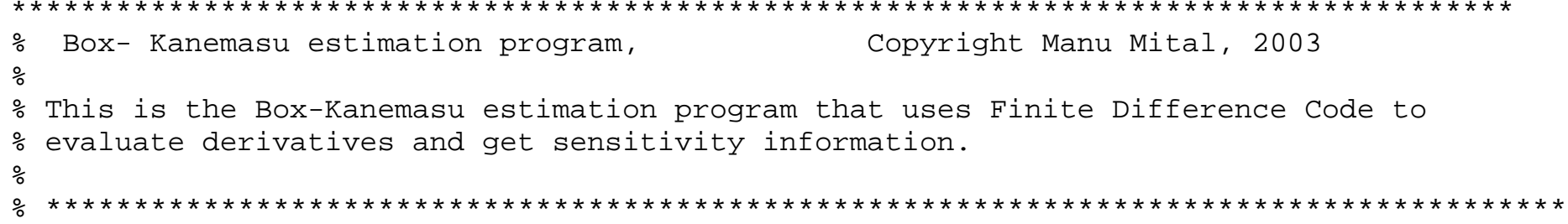




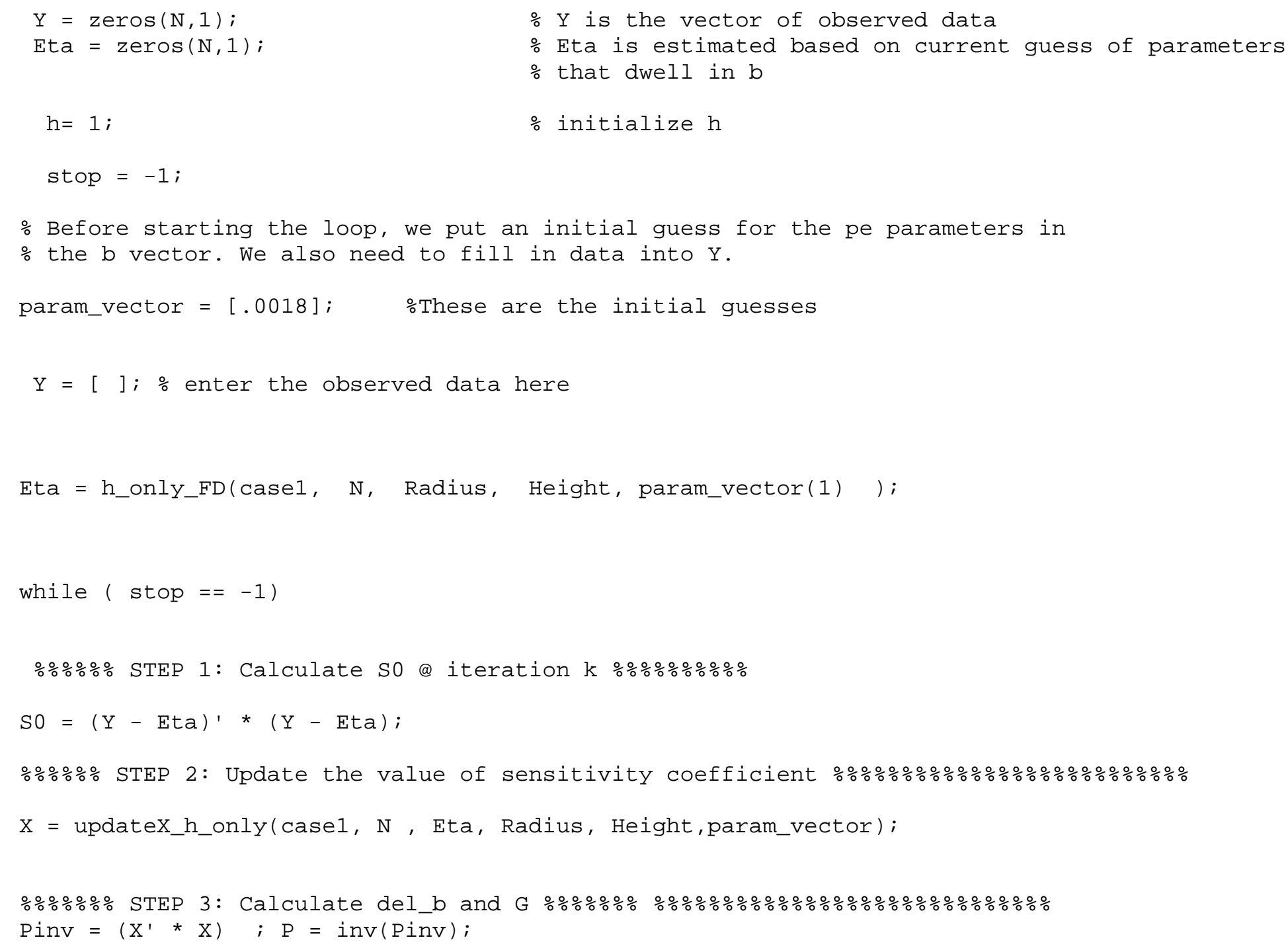


del_param_vector $=P *\left(X^{\prime} *(Y-E t a)\right) ;$

G = del_param_vector' * Pinv * del_param_vector ;

if $(\mathrm{G}<\odot)$ stop $=1$; end

\%\%\%\% STEP 4: Set the values of alpha and A \%\%\%\%\%\%\%\%\%\%\%\%\%\%\%\%\%\%\%

alpha $=\mathrm{h} ; \quad$ \% alpha is inititially set equal to 1

A $=1.1$

$\% \% \%$ STEP 5: Update the value of $\mathrm{b}$ that holds the current guess of parameters $\% \% \%$

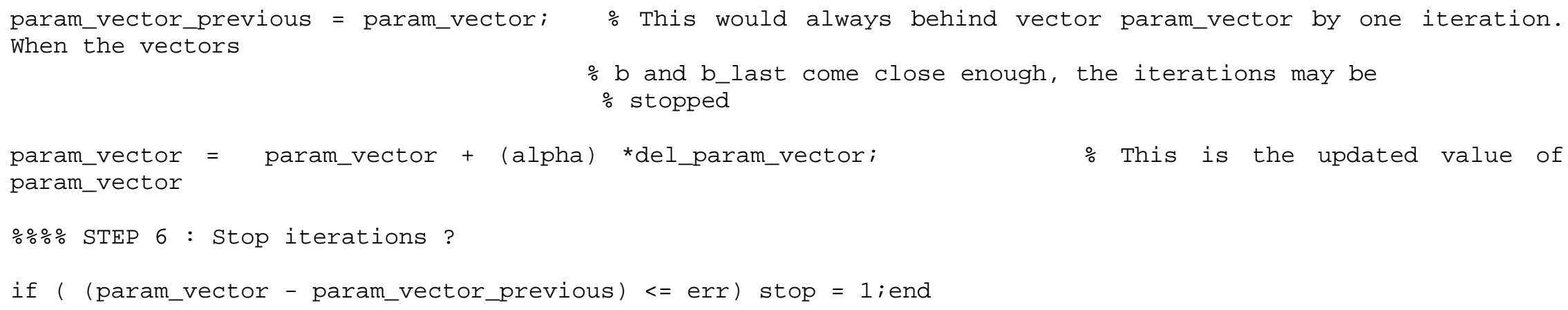

$\% \%$ STEP 6 : Stop iterations ?

if $($ (param_vector - param_vector_previous $)<=$ err $)$ stop $=1$; end

\%\%\% STEP 7: Calculate S_alpha using $\mathrm{b}$ that was updated in last step \%\%\%\%\%\%\%\%\%\%\%\%\%\%\%\%

Eta $=$ h_only_FD (case1, N, Radius, Height, param_vector(1));

S_alpha $=(Y-E t a)^{\prime} *(Y-E t a) ;$

S_check $=$ SO $-($ alpha * G $) *(2-(1 / A))$;

\%\%\%\% STEP 8 : Compare S_alpha with S_check and set the new value of $\mathrm{h} \% \% \% \% \% \% \% \% \% \% \% \% \%$ 
if $($ S_alpha $>=$ S_check $) h=(($ alpha^2) * G) / (S_alpha - S $\odot+2 * a l p h a * G)$;

else $\mathrm{h}=$ alpha $* \overline{\mathrm{A}}$; end

end $\%$ end of the while loop

param_vector \% print b

cputime-t

function $[\mathrm{x}]=$ Updatex (case1,N, eta,Radius, Height, param_vector $)$;

\section{UpdateX Function}

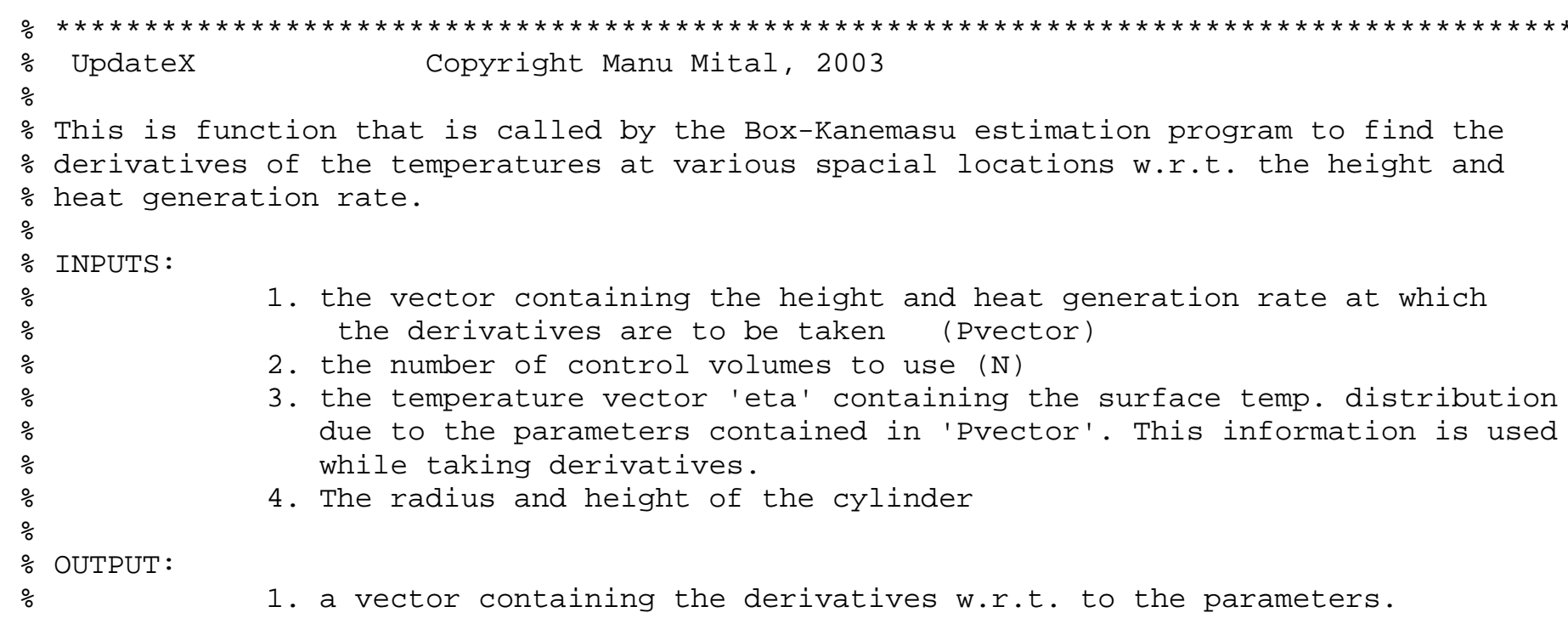

This is function that is called by the Box-Kanemasu estimation program to find the $\%$ derivatives of the temperatures at various spacial locations w.r.t. the height and $\%$ heat generation rate.

$\%$ INPUTS:

1. the vector containing the height and heat generation rate at which

$\%$ the derivatives are to be taken (Pvector)

$\% \quad 2$. the number of control volumes to use (N)

$\%$ 3. the temperature vector 'eta' containing the surface temp. distribution
$\%$
due to the parameters contained in 'Pvector'. This information is used while taking derivatives.

4. The radius and height of the cylinder

OUTPUT:

1. a vector containing the derivatives w.r.t. to the parameters. 
$\%$ Pvector $=$ [radius; height from base; heat generation rate $]$

$\mathrm{x}=\operatorname{zeros}(\mathrm{N}, 1) ; \%$ initialize the sensitivity coefficient vector

param_vector_modified $=$ param_vector $;$ param_vector_modified $(1)=$ param_vector_modified $(1)+.1 \mathrm{e}-4$;

F_del_conv $=$ h_only_FD (case1，N， Radius, Height, param_vector_modified(1) );

$x(:, 1)=\left(F \_\right.$del_conv - eta $) / .1 \mathrm{e}-4$; 


\section{Appendix F: Genetic Algorithm code}

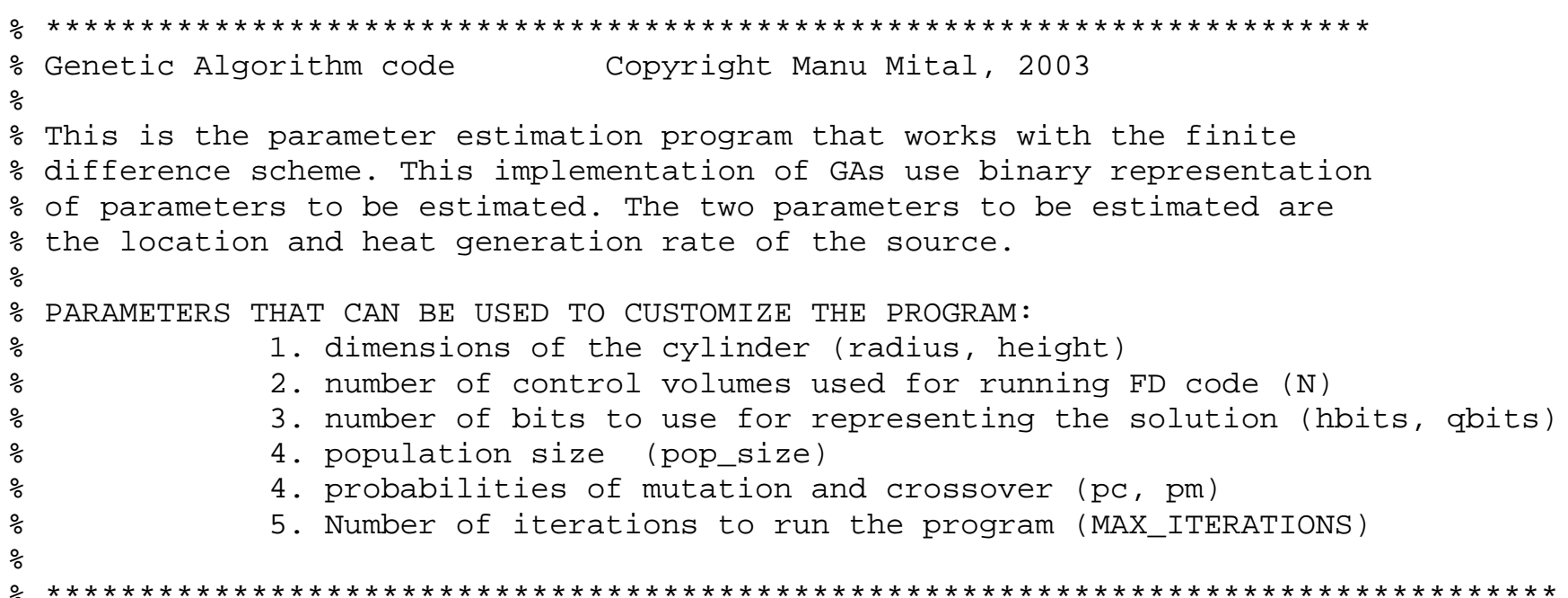

clear all; clc;

tic

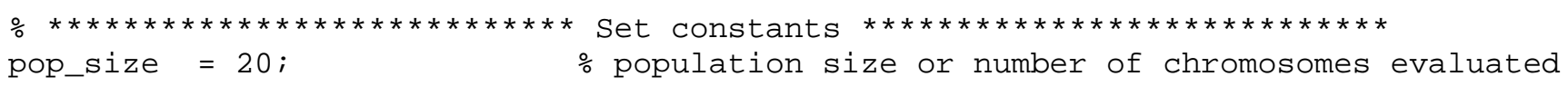

$\%$ two parameters of interest - height and heat generation rate

hbits $=5 ; \quad \%$ number of bits for the height of the tumor

qbits $=5 ; \quad \%$ number of bits for the heat generation rate

bits = hbits + qbits; $\quad \%$ Number of bits for each member (number of genes for each chromosome) 


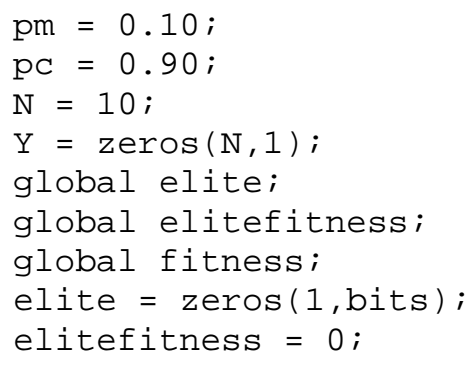


$\% * * *$ Crossover Code section

$p=0 ; \%$ crossovers on the population (initializing $p$ )

for $i=1:$ pop_size

$\%$ This loop generates a random number for each member which determines

$\%$ (when compared to the probability of crossover) whether or not crossover

$\%$ will occur in that member.

if rand < pc \% pc is the probability of crossover

$p=p+1 ; \quad \%$ steps through matrix

crossovers $(p)=i ; \%$ stores which member will crossover

end

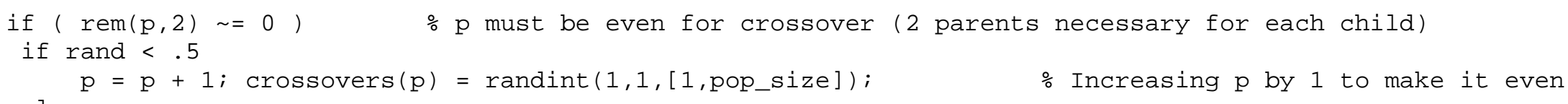




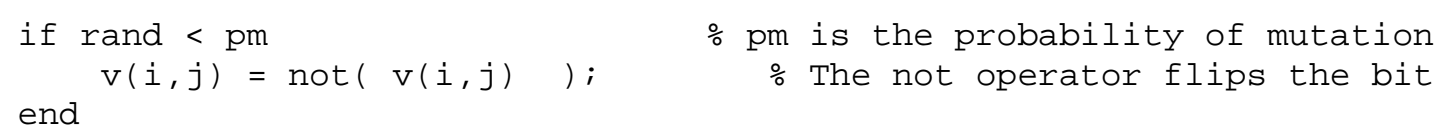

\section{\% Evaluate fitness of each chromosome}

for $i=1$ :pop_size

$\mathrm{b}=$ EvalChromosome ( $\mathrm{v}(\mathrm{i}, \mathrm{:})$, hbits, qbits, radius, height $) ; \quad$ \% constains [height; heatgen]

fitness $(i)=$ EvalFitness $(b, N, Y$, radius, height $)$;

$\%$ if $(30-$ fitness $(i)<=1 e-4$ \&\& terminate $==0)$ terminate $=1 ; \quad b$

end

$\%$ end

\% make a record of best possible solution so far.

$[$ FitVal, index $]=\max ($ fitness $)$;

if(FitVal > elitefitness) \% change the record of elite fitness

elitefitness = Fitval:

elite $=\mathrm{v}($ index,$:)$;

Elitesolution = EvalChromosome (elite, hbits, qbits, radius, height )

elseif (Fitval < elitefitness) \% inject the elite member into the population

sub = randint $(1,1,[1$, pop_size $])$;

$\mathrm{v}(\mathrm{sub},:)=$ elite;

fitness (sub) = elitefitness;

end 


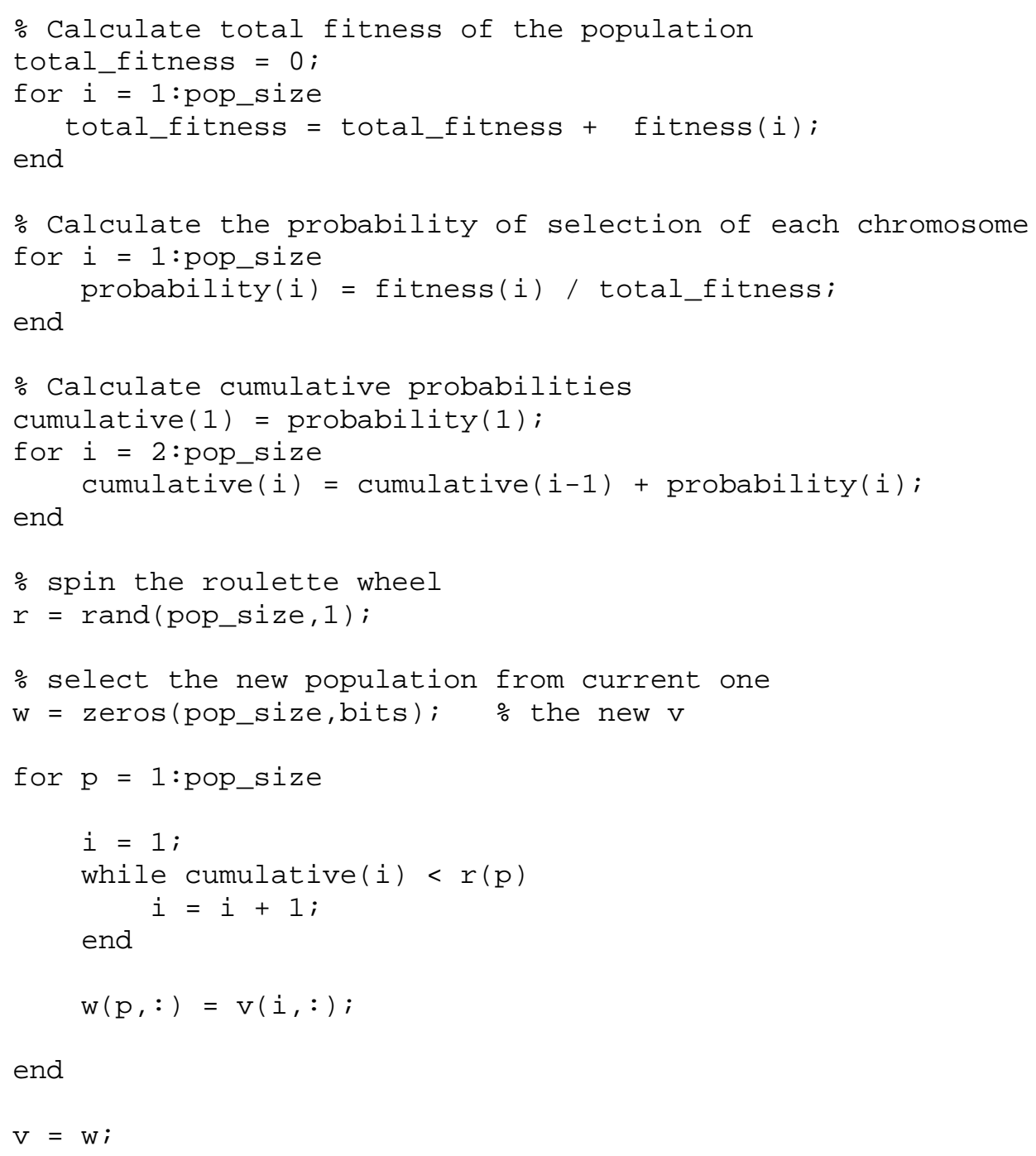

end

$\mathrm{v}=\mathrm{w}$; 
$\%$ If fitness does not change much or number of iterations >= MAX, terminate loop. 0therwise, continue. iterations $=$ iterations +1

if (iterations >= MAX_ITERATIONS)

terminate $=1$;

end

end

$\%$ !!!!!!! Main loop ends !!!!!!!!

$\%$ Output the results only if termination was due to all iterations completed if (iterations >= MAX_ITERATIONS)

$[$ FitVal, I] $=\max ($ fitness $)$;

$\mathrm{b}=$ EvalChromosome ( $\mathrm{v}(\mathrm{I}, \mathrm{:})$, hbits, qbits, radius, height ) ;

Elitesolution = Evalchromosome (elite, hbits, qbits, radius, height )

end

$\mathrm{t}=\mathrm{toc}$

\section{Fitness Evalaution Function}

function $[f]=$ EvalFitness $(b, N$, Obs, Rad, Ht);

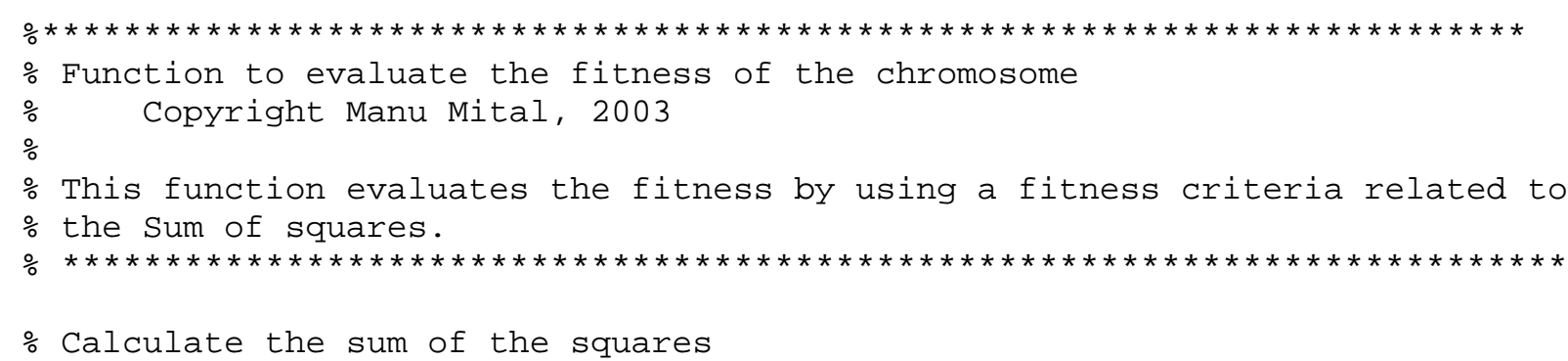


Eta $=$ cylindrical_SS_2D $(b, N$, Rad, Ht $)$;

$S=(\text { Obs }- \text { Eta })^{\prime} *$ (Obs - Eta);

$f=200-10 * S$;

\section{Crossover Function}

function $[\mathrm{x}, \mathrm{y}]=\operatorname{Docrossover}(\mathrm{a}, \mathrm{b}, \operatorname{nbits})$;

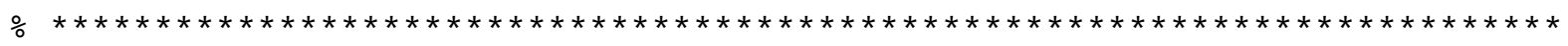

$\%$ Crossover function Copyright Manu Mital, 2003

$\%$

$\%$ This function takes two chromosomes a and b. It also takes in the number

$\%$ of bits in the chromosome. It then does crossover by using a random

$\%$ number as the crossover location. The function returns the two new

$\%$ chromosomes created as a result of crossover operation.

$\%$

$r=\operatorname{randint}(1,1,[1$, nbits $])$;

for $i=r+1:$ nbits

if $(a(1, i) \sim=b(1, i))$ Exchange bits if they are different $a(1, i)=\operatorname{not}(a(1, i))$;

end

$b(1, i)=\operatorname{not}(b(1, i))$;

\section{end}

$\mathrm{x}=\mathrm{a} ; \mathrm{y}=\mathrm{b}$; 


\section{Appendix G: Infrared Data Reduction Program}

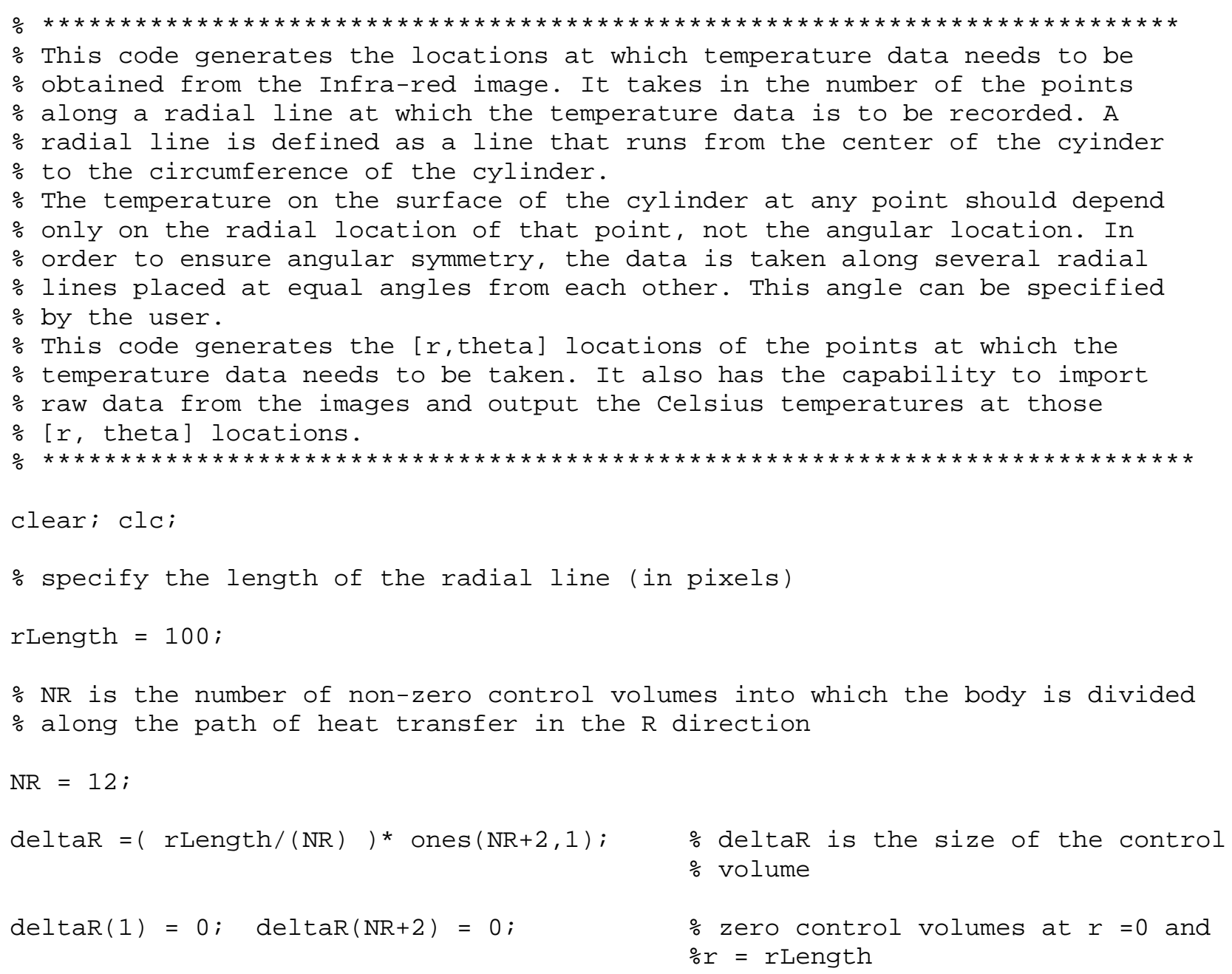


\% Compute the values of locations along radial lines at which the temperature $\%$ data will be taken

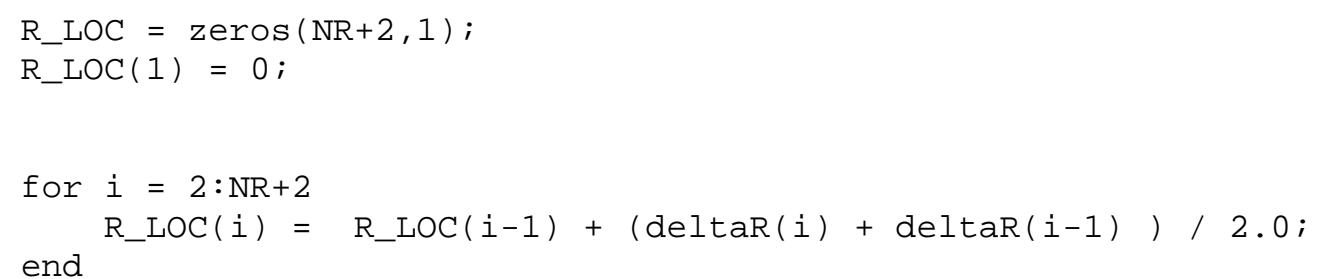


y_pos $=$ zeros $($ nLines, NR);

for $i=1: 1:$ nLines

for $j=1: 1: N R$

$x \_p o s(i, j)=r(j) * \cos ($ theta * pi / 180);

$y \_\operatorname{pos}(i, j)=r(j) * \sin ($ theta $* p i / 180)$;

end

theta $=$ theta + ang;

end

$\%$ Since the $[r$, theta] coordinate system and the $[x, y]$ coordinate system

$\%$ do not share the same origin, we need to offset the points in $x$ _pos and

\% y_pos matrices

\% First specify the center of the $[r$, theta] system

center_x = 162; center_y = 123;

\% Now add these to the x_pos and y_pos matrices

x_pos $=$ x_pos + center_x * ones $($ nLines, NR $)$;

y_pos $=$ y_pos + center_y * ones $(n L i n e s, \quad N R)$;

x_pos $=$ round $\left(x \_p o s\right)$;

$y_{-}$pos $=\operatorname{round}\left(\mathrm{y}_{-}\right.$pos $) ;$

$\% \mathrm{x}$ _pos and $\mathrm{y}$ _pos now contain the $\mathrm{x}$ and $\mathrm{y}$ coordinates of the locations at which

$\%$ the temperature data is needed. 
$\%$ Load the file having array of greyscale values

load data.txt;

data_matrix = data $(:,:)$;

for $i=1: n L i n e s$

for $j=1: N R$

raw_temp $(i, j)=$ data_matrix $\left(y \_p o s(i, j) \quad, \quad x \_p o s(i, j) \quad\right)$;

end

end

$\%$ The raw temperaturematrix contains the greyscale temperature values. To

$\%$ convert these values to celsius temperatures, we use equation of the form

$\%$ celsius temp $=$ raw temp * $m+b$

$\%$ calibration data

$\%$ pRaw $=\left[\begin{array}{lllll}1 & 2 & 3 & 4 & 5\end{array}\right] ;$ pCelsius $=\left[\begin{array}{lllll}1 & 2 & 3 & 4 & 5\end{array}\right] ;$

\%calibration $=$ polyfit $($ pRaw, pCelsius, 1$) ;$

calibration $=[.0056,2.36]$;

celsius_temp $=$ raw_temp * calibration $(1)+$ calibration( 2$)$;

$\%$ we now calculate the mean temperature of each location and the standard deviation $\%$ of the temperature at that location.

for $j=1: N R$ 
mean_temp $(j)=$ mean $\left(\operatorname{celsius\_ temp}(:, j)\right)$;

std_temp $(j)=\operatorname{std}($ celsius_temp $(:, j))$;

end

\% celsius temperatures are the temperatures in celsius at the specified $x$ $\%$ and y locations.

[mean_temp', std_temp'] 


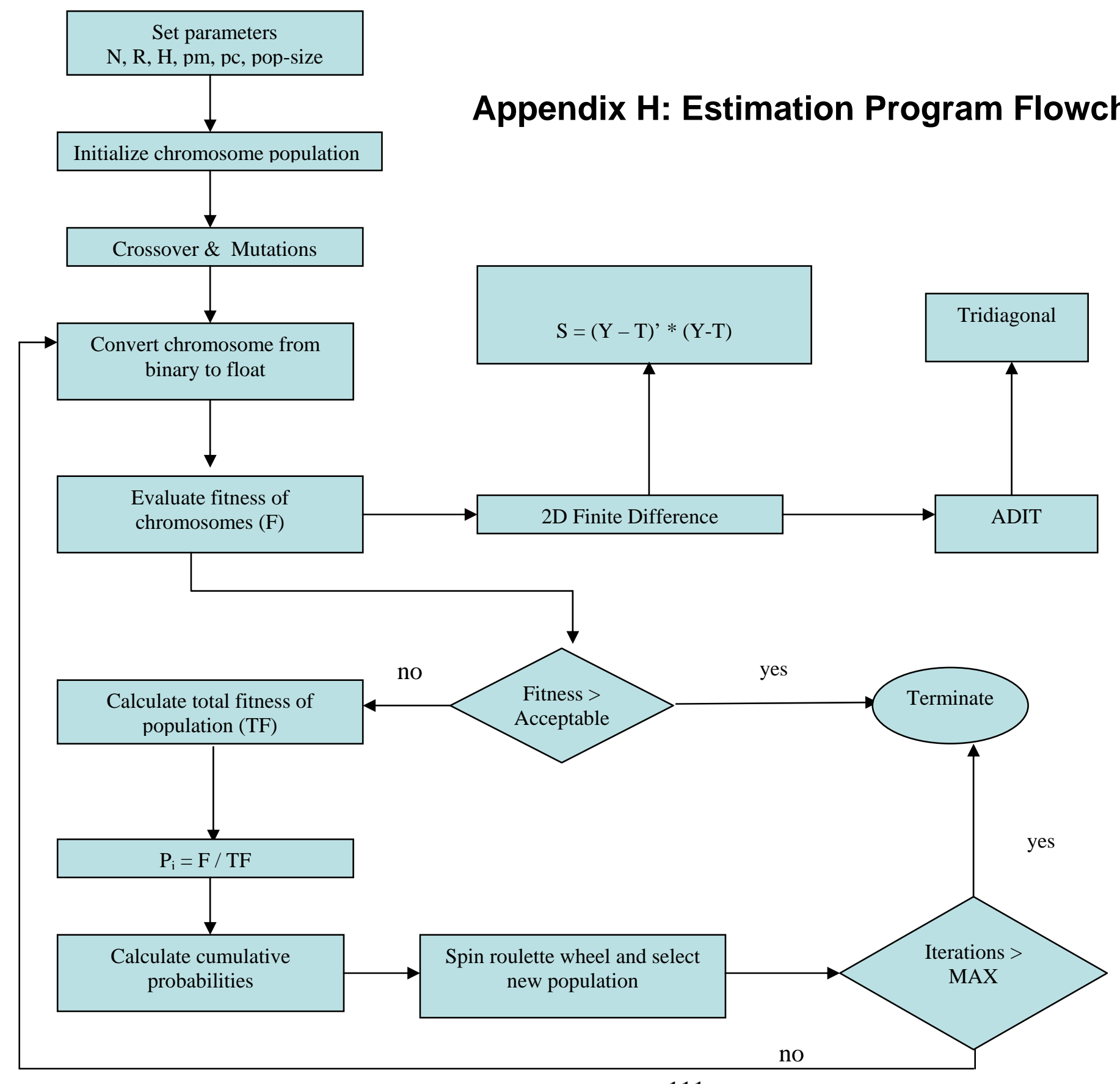




\section{Appendix I: Infrared Camera Specifications}

Table I.1 Specifications for the PV320 Infrared Camera

\begin{tabular}{|c|c|}
\hline $18 \mathrm{~mm}$ & f 1.0 Field of view $52^{\circ}$ \\
\hline Field/Frame Rate & $60 / 30 \mathrm{~Hz}$ \\
\hline Sensor Type & Uncooled Ferroelectric \\
\hline Size & 140 mm (L) x 114 mm (W) x $114(\mathrm{H})$ \\
\hline Temperature Calibration Range & $-20^{\circ}-500^{\circ} \mathrm{C}$ \\
\hline 25MM & f 1.0 Field of View $35^{\circ}$ \\
\hline Array Resolution & $320 \times 240$ \\
\hline Digitization & 12-bit \\
\hline Measurement Accuracy & $+/-2 \%$ \\
\hline Weight & 3 lbs. without lens \\
\hline $35 \mathrm{~mm}$ & f 1.0 Field of View $25^{\circ}$ \\
\hline Ambient Operating Temperature & $-20^{\circ} \mathrm{C}$ to $50^{\circ} \mathrm{C}$ \\
\hline Digital Output & USB 2.0 High Speed \\
\hline Spectral Response & 8-14 Microns (2-14 Microns - Optional) \\
\hline $50 \mathrm{MM}$ & f 1.0 Field of View $18^{\circ}$ \\
\hline Storage Temperature & $-20^{\circ} \mathrm{C}$ to $60^{\circ} \mathrm{C}$ \\
\hline Video Output & NTSC or PAL \\
\hline $100 \mathrm{~mm}$ & f 1.0 Field of View $9^{\circ}$ \\
\hline NETD & $80 \mathrm{mK}$ \\
\hline Remote Control & RS-232 \\
\hline Tripod Mount & 1/4-20 Standard \\
\hline Detector Cooling & TE Stabilized \\
\hline TE Stabilized & 47 microns \\
\hline
\end{tabular}




\section{Vita}

Manu Mital was born in New Delhi, India. In May 2003, he received his degree in Bachelor of Science in Mechanical Engineering at Virginia Tech, Blacksburg, Virginia. During the summer of 2003, he worked as a Packaging Engineering Intern at Fres-co Systems USA. His work involved designing, assembling and installing mechanical assemblies for packaging machinery, maintaining materials database, and drafting AutoCAD drawings. In August 2003, he started his master's research at Virginia Tech in the field of heat transfer and optimization, working under the supervision of Dr. Elaine Scott. He received his Master of Science in Mechanical Engineering in December 2004. Manu Mital is currently pursuing his doctorate degree in Mechanical Engineering at Virginia Tech. 